\title{
Contaminant-Induced Current Decline in Capillary Array Electrophoresis
}

\author{
By
}

Robin Coope

BASc., The University of British Columbia 1993

MASc., The University of British Columbia 1996

A THESIS SUBMITTED IN PARTIAL FULFILLMENT OF

THE REQUIREMENTS FOR THE DEGREE OF

\author{
DOCTOR OF PHILOSOPHY \\ in \\ The Faculty of Graduate Studies \\ (Physics)
}

THE UNIVERSITY OF BRITISH COLUMBIA

April 2006

(C) Robin Coope,2006 


\section{Abstract}

This research clarifies, for the first time, the mechanism and impact of current decline in capillary array electrophoresis (CAE). High throughput capillary array electrophoresis instruments for DNA sequencing suffer to varying degrees from failure associated with electrophoretic current decline and inhibition or delay in the arrival of fragments at the detector. This effect is known to be associated with residual quantities of large, slow moving fragments of template or genomic DNA carried through from sample preparation and sequencing reactions. Here, we document and investigate the existence of an expanding ionic depletion region induced by overloading the capillary with low-mobility DNA fragments, and the effect of growth of this region on electrophoresis run failure.

This depletion region forms upstream of the smaller sequencing fragments, and its expansion was found not to affect the quality of the sequencing peaks at the detector. Rather the current decline associated with depletion region growth reduces the velocity of the downstream sequencing fragments, so fewer fragments arrive at the detector during the run.

It is shown, through analytical and numerical models, how increasing quantities of slow moving DNA cause the concentration of background electrolyte downstream to decline. With the concentration of such fragments beyond a threshold quantity, the anode-side boundary of the nascent depletion region is shown to propagate toward the anode at a rate faster than the contaminant DNA migration. Under such conditions the depletion region expands, the current declines, and the electrophoresis run suffers from a reduced yield of sequence data or fails completely. 
While the upstream boundary of the depletion region propagates with the DNA, the propagation rate of the downstream boundary is found to be inversely proportional to the amount of ionic depletion, and independent of the motion of the DNA. Observations suggest that these downstream boundaries may propagate as a result of an imbalance in current carriers brought about by the exposure of bound charges in the matrix or capillary wall, which may be coupled to a rise in $\mathrm{pH}$. 


\section{Table of Contents}

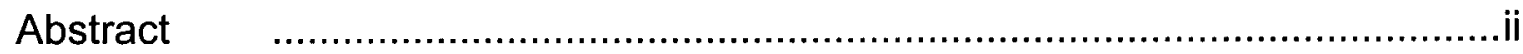

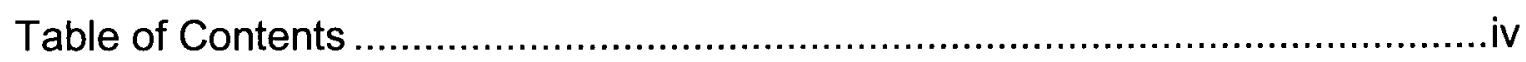

List of Tables ................................................................................ vii

List of Figures ........................................................................... vii

Symbols, Nomenclature and Abbreviations ............................................

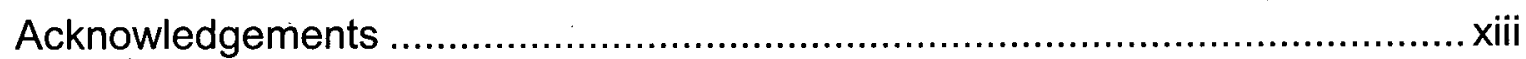

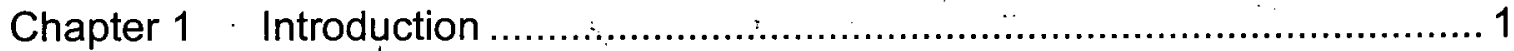

1.1 The Continued Importance of de novo Sequencing and Capillary

Electrophoresis .................................................................. 1

1.2 Matrix selectivity is the next parameter to push for increased

performance..................................................................... 3

Chapter 2 The Principles of Capillary Electrophoresis............................... 5

2.1 Principles of Electrophoresis....................................................... 5

2.2DNA Propagation in Matrices................................................. 8

2.3Other Properties of Electrophoresis ........................................... 9

2.4 Electrokinetic Injection and Sample Stacking :.................................. 11

2.5Capillary Sequencing for DNA .............................................. 13

2.6Current Decline and Mitigation Efforts ......................................... 19

Chapter 3 Apparatus and Methods to Investigate Current Decline............... 22

3.1The MegaBACE ............................................................. 24

3.2The Single Capillary Instrument................................................ 26

3.2.1 Mechanical Design................................................ 26

3.2.2 Fluorescence Detection ......................................... 30

3.2.3 Thermal and Visible Light Cameras .............................. 32

3.2.4 Capillaries, Matrix and Buffer .................................... 33

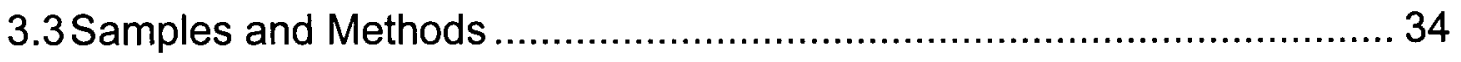

3.3.1 MegaBACE Samples ........................................... 34 
3.3.2 MegaBACE Experimental Parameters ................................. 35

3.3.3 Electropherogram Analysis ............................................ 35

3.3.4 Single Capillary Instrument Samples ................................ 38

3.3.5 Single Capillary Instrument Parameters............................. 40

3.3.6 Cut Capillary and Thermal Image Analysis .......................... 41

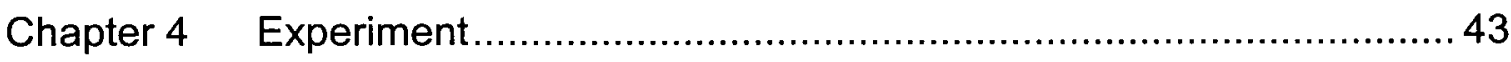

4.1 Relationship between read failure and capillary current decline............. 43

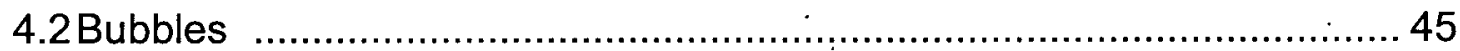

4.2.1 The Contribution of Bubbles to Current Decline ................... 45

4.2.2 Bubble Formation and Growth ....................................... 48

4.3 Ion Distribution during Current Decline ................................................. 49

4.3.1 Formation of a Depletion Region.......................................... 49

4.3.2 A Threshold for Current Decline ........................................... 53

4.4Temporal evolution of Ion Depletion Boundaries.................................. 54

4.4.1 Cathode Boundary and DNA Movement .............................56

4.4.2 Boundary velocity and its relation to depletion region

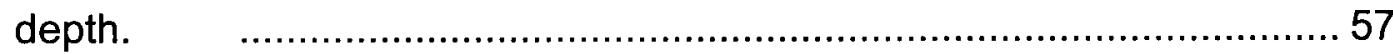

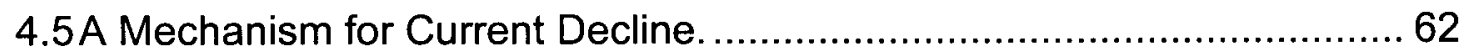

Chapter 5 Analysis of Boundary Propagation ............................................ 64

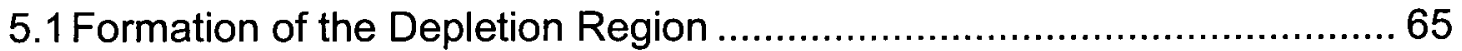

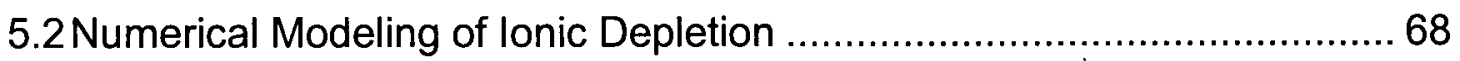

5.3DNA and Cathode Boundary Propagation ............................................ 80

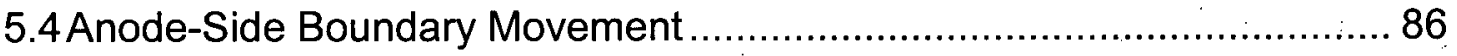

5.4.1 The Theory of Moving Boundaries ....................................... 86

5.4.2 Common Ion Boundaries ................................................... 91

5.4.3 Boundary Movement by Bound Charge ............................... 95

5.4.4 Experimental Evidence for $\mathrm{pH}$ Changes in the

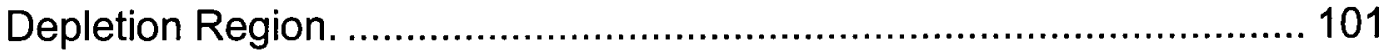

5.4.5 Boundary Movement by $\mathrm{OH}^{-}$Propagation.......................... 106

Chapter 6 Conclusion .......................................................................... 111 


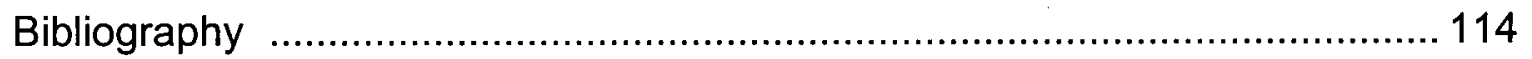

Appendix A Properties of DNA and DNA Sequence Methods...................... 125

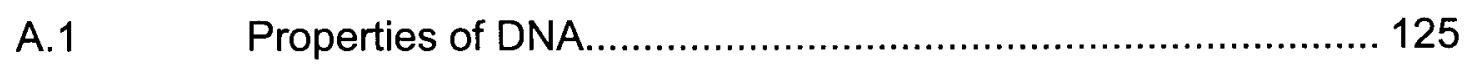

A.2 Library Construction ….................................................. 126

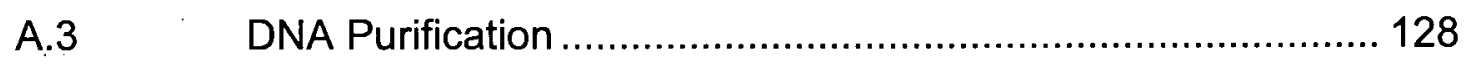

A.4 Cycle Sequencing....................................................... 129

A.5 BC Genome Sciences .Center Sample Preparation ............... 131

Appendix B The Effect of Sample Resuspension in Agarose ....................... 134

B.1 Effects of Injection from Agarose.......................................... 134

Appendix C Secondary Effects of Ionic Depletion and Bubble

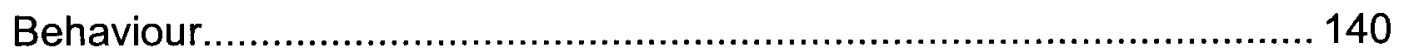

C.1 Permanent Effects of DNA-Induced Depletion Regions. ......... 140

C.2 Matrix ion depletion from anode-side buffer. ......................... 146

C.3 Bubbles' Effect on Current................................................. 150

Appendix D LPA and Buffer Properties ................................................. 156

D.1 Conductivity and pH of Tris/TAPS Buffer Under Dilution ........ 156

D.2 The Thermal Expansion Coefficient of LPA.......................... 158

Appendix E Temperature Measurement.................................................. 160 


\section{List of Tables}

Table 1 Data for concentration and solution conductivity for different DNA samples...

Table 2 Physical data for the numerical model................................ 75

Table 3 Signal strength of the first $\sim 50$ DNA bases in capillaries resuspended in DI H2O and Agarose ............................... 138 


\section{List of Figures}

Figure 1 Schematic description of electrophoretic properties of DNA,

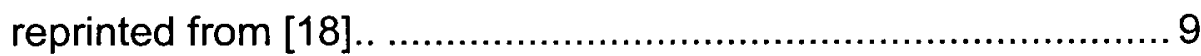

Figure 2 The original confocal array developed by the Mathies group.

Reprinted from [35] 15

Figure 3 The airbox in the MegaBACE showing the capillary array, anode pressure vessel and cathode array ............................. 24

Figure $4 \mathrm{~A}$ front view schematic of the single capillary sequencer.....26

Figure $5 \mathrm{~A}$ schematic of the confocal fluorescence detector system. 30

Figure 6 MegaBACE electropherogram data.................................. 37

Figure 7 A typical successful electropherogram. ............................. 38

Figure 8 Read length vs total loaded charge.................................. 44

Figure 9: Total loaded charge of capillaries with and without bubbles.

Figure 10 Local conductivity and resistance profiles for four cut capillaries.

Figure 11 Current at the end of runs of varying duration inferred from ionic concentration profiles. 53

Figure 12 Total loaded charge vs. quantity of injected DNA 54

Figure $13 \mathrm{~A}$ series of infrared images of the capillary taken eight seconds apart and displayed side by side. 56

Figure 14 Fits to the first five minutes of anode and cathode boundary data using $x(t)=C_{1} Q(t)+C_{2}$ 58

Figure 15 Two examples of ionic depletion boundaries propagating in from a low conductivity cathode. 60

Figure 16 Measured propagation rates for boundaries of different depletion depths. 
Figure 17 Rate of anode-side boundary propagation vs. quantity of injected DNA

Figure 18 1-D discretization of the capillary showing ion flows in the upwind scheme.

Figure 19 Decay of a peak in ion concentration due to numerical diffusion.

Figure 20 Effect of a fixed DNA peak on the background electrolyte concentration 78

Figure 21 The effect of a $20 \mathrm{ng}$ DNA peak of varying width. 79

Figure 22 Fit of equation (5-29) to a cathode boundary position........ 84

Figure 23 Adjacent ionic regions forming a moving boundary. 87

Figure 24. The effect of the regulating function on the distribution of ions in the capillary 89

Figure 25 Simulation of a leading and trailing negative ion. 90

Figure 26 Common ion boundary motion in buffered and unbuffered systems. .94

Figure 27: Bound charge driven boundary movement. .98

Figure 28 Comparison of experimental data and simulation of

boundary propagation due to bound charges. 99

Figure $29 \mathrm{pH}$ details of bound charge driven depletion regions....... 100

Figure 30 Nickel particles in a capillary crossing into the depletion region. 103

Figure 31 Progress of a nickel particle as a depletion boundary crosses its path. 104

Figure 32 A five minute simulation with [TAPS] $=0$ at cathode. The retreating TAPS ${ }^{-}$ion is replaced by $\mathrm{OH}^{-}$ions, raising the $\mathrm{pH} .108$

Figure 33 Comparison of simulation and experiment for $\mathrm{pH}$ driven boundaries. 109

Figure 34 Read length vs total loaded charge for samples resuspended in $\mathrm{dH}_{2} \mathrm{O}$ and agarose 135 
Figure 35 Electropherograms of $1 \mathrm{~kb}$ ladder resuspended in $\mathrm{DI} \mathrm{H}_{2} \mathrm{O}$ and $0.08 \%$ Agarose 137

Figure 36. Infrared images (left) of a surface charge induced depletion region (SCIDR) . 141

Figure 37 Creation of a SCIDR by addition of $\lambda$ DNA 142

Figure 38 The effect of clipping off the capillary entrance on the movement of a SCIDR.

Figure 39: Current decline associated with using buffer at the anode. 146

Figure 40 Conducitivity changes from matrix depletion 147

Figure 41 Effect of a SCIDR boundary meeting a matrix depletion boundary 150

Figure 42: Infrared and visible images of the capillary showing simultaneous growth of a hot region and bubble. 151

Figure $43 \mathrm{~A}$ histogram showing observed bubble positions after one run. 152

Figure 44. A histogram of the data in Figure 8(a), binning the capillaries by total loaded charge. 154

Figure 45 Current traces from one plate from the MegaBACE. ..... 155

Figure 46 Conductivity of the Tris-TAPS buffer.. 157

Figure 47 Temperature dependence of $\mathrm{pH}$ and conductivity in $50 \mathrm{mmol} / \mathrm{L}$ Tris-TAPS. 158

Figure $48 \mathrm{~A}$ thermal of the capillary and temperature references.... 160

Figure 49 Thermal maps of the capillary depletion region. 162

Figure 50 The rise in temperature in the depletion region over the course of a run 163 


\section{Symbols, Nomenclature and}

\section{Abbreviations}

\begin{tabular}{|c|c|}
\hline Term & Definition \\
\hline $\mathrm{F}$ & Faraday's Constant: 96800 coulombs/mol \\
\hline$\sigma$ & Conductivity of a solution, $(\Omega \mathrm{m})^{-1}=10^{-4} \mu \mathrm{S} / \mathrm{cm}$ \\
\hline$\mu_{\mathrm{i}}$ & Ionic mobility of the ith species $\left(\mathrm{m}^{2} \mathrm{Ns}\right)$ \\
\hline $\mathrm{C}_{\mathrm{i}}$ & $\begin{array}{l}\text { Concentration of the ith species. } 1 \mathrm{~mol} / \mathrm{m}^{3}= \\
1 \mathrm{mmol} / \mathrm{L}\end{array}$ \\
\hline $\mathrm{S}$ & Siemens $=\Omega^{-1}$ \\
\hline $\mathrm{e}$ & Elementary charge $1.6 \times 10^{-19} \mathrm{C}$ \\
\hline $\mathrm{J}$ & Current density $\left(\mathrm{Amps} / \mathrm{m}^{2}\right)$ \\
\hline 1 & Current (Amps) \\
\hline$v$ & Ion velocity $(\mathrm{m} / \mathrm{s})$ \\
\hline Q & Charge (coulombs) \\
\hline$t_{r}$ & Run time (s) \\
\hline FWHM & Full width-half maximum of a gaussian peak. \\
\hline $\mathrm{D}$ & Diffusion constant $\left(\mathrm{m}^{2} / \mathrm{s}\right)$ \\
\hline$\lambda$ DNA & A 48kb double stranded non-circular phage DNA. \\
\hline Phred & $\begin{array}{l}\text { The most common algorithm for identifying DNA } \\
\text { bases from raw fluorescent sequence data. [1] }\end{array}$ \\
\hline Read length & $\begin{array}{l}\text { The number of contiguous nucleotides identified at } \\
\text { satisfactory quality, typically Phred quality }>20 \text {, in } \\
\text { a single sequence run. (see Phred) }\end{array}$ \\
\hline Aliquot & To distribute volume of fluid to multiple sites \\
\hline Phage & $\begin{array}{l}\text { A virus, including a DNA strand, that infects } \\
\text { bacteria }\end{array}$ \\
\hline Plasmid & $\begin{array}{l}\text { A circular DNA strand that can reproduce in } \\
\text { bacteria }\end{array}$ \\
\hline Vector & A more general term for a plasmid or phage. \\
\hline Insert & DNA of interest ligated into a vector \\
\hline $\mathrm{dH}_{2} \mathrm{O}$ & Deionized water $\sigma=0.05 \mu \mathrm{S} / \mathrm{cm}$ \\
\hline TLC & Total Loaded Charge \\
\hline $\begin{array}{l}\text { Transference } \\
\text { Number }\end{array}$ & $T_{-}=\frac{\mu_{-}}{\mu_{-}+\mu_{+}}, T_{+}=1-T_{-}$ \\
\hline Electropherogram & Raw data from an electrophoresis machine. \\
\hline Anode & $\begin{array}{l}\text { The positive end of the capillary electrophoresis } \\
\text { apparatus }\end{array}$ \\
\hline
\end{tabular}




\begin{tabular}{|c|c|}
\hline Cathode & $\begin{array}{l}\text { The negative end of the capillary electrophoresis } \\
\text { apparatus }\end{array}$ \\
\hline Electrophoresis & $\begin{array}{l}\text { The process of ions migrating through solution in } \\
\text { response to an electric field. }\end{array}$ \\
\hline Co-ion & An ion of like charge \\
\hline Counter ion & An ion of opposite charge \\
\hline$\% w / v$ & $\begin{array}{l}\text { Percent weight/volume (usually } \mathrm{g} / \mathrm{ml} \text { ). This is a } \\
\text { literature convention for polymer concentrations. }\end{array}$ \\
\hline LPA & $\begin{array}{l}\text { Linear Polyacrylamide - the DNA separation } \\
\text { polymer used in this research }\end{array}$ \\
\hline PDMA & $\begin{array}{l}\text { Polydimethylacrylamide. A popular polymer for } \\
\text { DNA separations. }\end{array}$ \\
\hline$\overline{P C R}$ & $\begin{array}{l}\text { Polymerase Chain Reaction - the protocol used to } \\
\text { amplify DNA fragments by up to } 10^{6} x\end{array}$ \\
\hline $\mathrm{ABI}$ & $\begin{array}{l}\text { Applied Biosystems Inc. Manufacturer of the family } \\
\text { of DNA sequencers }(310,3100,3700,3730) \text { which } \\
\text { dominates the market. }\end{array}$ \\
\hline LIF & Laser Induced Fluorescence \\
\hline BCGSC & The BC Cancer Agency Genome Sciences Center. \\
\hline Tris & $\begin{array}{l}\text { Tris(hydroxymethyl)aminomethane) The weak } \\
\text { base commonly used in DNA sequencing buffer }\end{array}$ \\
\hline TAPS & $\begin{array}{l}\text { [(2-Hydroxy-1,1-bis (hydroxymethyl)ethyl)amino]-1- } \\
\text { propanesulfonic acid. The weak acid commonly } \\
\text { used in DNA sequencing buffer }\end{array}$ \\
\hline PMT & $\begin{array}{l}\text { Photomultiplier Tube. The fluorescence detector } \\
\text { used in many capillary sequencers }\end{array}$ \\
\hline In silico & $\begin{array}{l}\text { A term, found in life science, meaning "using a } \\
\text { computer". It follows from in vivo and in vitro }\end{array}$ \\
\hline EOF & Electro-osmotic flow. \\
\hline TSR & $\begin{array}{l}\text { Template Suppression Reagent - an additive to } \\
\text { prevent large DNA fragments from being co- } \\
\text { injected with sequencing samples }\end{array}$ \\
\hline $\mathrm{bp} / \mathrm{kbp} / \mathrm{Mbp}$ & $\begin{array}{l}\text { Measurement of the length of a DNA strand in } \\
\text { number of base pairs, or kilo or mega base pairs. }\end{array}$ \\
\hline $\mathrm{CE}$ & Capillary Electrophoresis \\
\hline CZE & $\begin{array}{l}\text { Capillary Zone Electrophoresis. The broader } \\
\text { category of electrophoresis which covers DNA } \\
\text { separations }\end{array}$ \\
\hline
\end{tabular}




\section{Acknowledgements}

I am indebted to numerous people who made my $\mathrm{PhD}$ possible. First amongst these is, of course, Andre Marziali, whose support, guidance and commitment was, and is, second to none. The absolute master of the rapid and critical insight, Dr. Marziali was able to help the research forward when I got stuck, keep it going in the right direction, and provide the ever-necessary optimism. Speaking of which, Dr. Marziali's development of the Physics 253 robotics course which I had the privilege to help teach will go down as the most fun anyone will ever have in a classroom. I challenge anyone to find a more effective, inspirational or generous thesis supervisor in any field anywhere.

Another great source of assistance was the staff of the BC Cancer Agency Genome Sciences Center and in particular, Duane Smailus. Duane prepared many of the samples used herein, always with dispatch, good humour and his usual standards of quality and consistency. I must thank my colleagues in the lab, and in particular second year co-op student Peter Eugster. Peter, some of whose data appears in this thesis, completed a large number of bubble and infrared experiments in a short period of time and remains my gold standard for efficient execution in the laboratory. Itinerant biochemist Trevor Pugh also provided samples, as did Mary Pines, late of the Sadowski lab. Co-op student Stefan Avall worked on numerical modeling early on, and together we certainly found some blind alleys to avoid! I must also thank fellow graduate students Jon Nakane and Matthew Wiggin for, respectively, their mathematical and biochemical insights and the general good times. Thanks also to the Tiedje lab crew of Anders Ballestad, Eric Nodwell and Scott Webster for letting me use Berserk and the Steamengine cluster, as well as the 
mathematical, Linux and Matlab insights. Thanks to Boye Ahlborn for translating parts of Kohlrausch's paper, written in the difficult classical scientific German of 1897. Thanks also to Barb Grossman, then of Amersham Biosciences, who ferreted out the object files that allowed me to properly analyse MegaBACE data. I am also grateful to my parents, John and Marian Coope, who in addition to everything else, read this thesis at the very end and found that last 300 or so errors. And of course Stacey Lobin, for being mom to Ben, who may be the most beautiful child ever, although it seems impossible to design an experiment to test this.

Finally I thank my thesis committee, Lorne Whitehead, Jeff Young and Matt Choptuik for taking the time to participate in this intellectual journey which I have so very much enjoyed. 
I am no poet, but if you think for yourselves as I proceed, the facts will form a poem in your minds.

Michael Faraday 


\section{Chapter 1 Introduction}

\subsection{The Continued Importance of de novo Sequencing and Capillary Electrophoresis}

As of the close of the twentieth century, large-scale DNA sequencing has emerged as a revolutionary scientific technique. Sequencing may come to be seen as akin to the development of the telescope or the particle accelerator in its impact on understanding of the world and our place in it. Data from the human genome project and other species' genomes have already begun to modify our understanding of the roles of genes and gene regulation and the regulatory role of non-coding DNA. Availability of genomes of multiple species has aided in locating genes associated with disease. More than one commentator has argued that biology is only now entering its golden era [2].

Genomics in the post-human genome project era is branching along several paths. One of these is the gathering of more species' genomes for comparative genomics, or comparison of sequence similarities between various organisms and human DNA, to increase our understanding of gene function, and of evolution of sequence information. Another recently embarked-upon endeavour is the sequencing of thousands of human cancer genomes to study cancer progression and the role of genetic mutations in this disease. Clearly these projects will require orders of magnitude more sequencing capacity than the entire human genome project, and will be limited by cost. Although the cost of sequencing has declined from $\$ 10 /$ base in 1990 [3] to about $\$ 0.004 /$ base in 2005 [4], a human-sized genome of $3 \times 10^{9}$ bases currently costs (with multiple coverage) about $\$ 50$ million. $\mathrm{NIH}$ director Francis Collins has called for the development of 
technologies for the " $\$ 1000$ Genome" or about $\$ 3 \times 10^{-7} / \mathrm{base}$.

Promising candidates thus far use fluorescently labeled DNA extension reactions [5] [6] [7] to generate short ( $<100$ base) continuous reads in a massively parallel manner. As a result, these methods are likely to be restricted to resequencing individual organisms for which there already exists a species genome to which the short fragments can be compared. De novo sequencing, on the other hand, requires the sequenced fragments be assembled into a continuous whole without the benefit of a reference sequence. Because of repeated sequence and other factors, the completeness of the assembled genome is dependent on the length of the fragments to a great degree [8], and large scale de novo sequencing is only practical with average read lengths of several hundred bases (see Appendix A.1).

Presently, and for the foreseeable future, the dominant technique for large-scale de-novo sequencing is capillary array electrophoresis (CAE, or CE). In CE, separation of DNA strands of different lengths is performed in an array of 96 or $38475 \mathrm{um} \mathrm{ID,} 60 \mathrm{~cm}$ long, discrete glass capillaries. This approach has a combination of advantages over on-chip CE or slab gel electrophoresis, including improved heat dissipation, long read lengths, speed, and ease of automation. In order to justify the continued commitment of resources at existing levels, the cost of de novo sequencing must continue to fall. In the context of $\mathrm{CE}$, this means lower reagent costs and longer read lengths from each capillary. As patent protection has maintained relatively high polymerase reagent costs, reaction dilution has recently been the biggest source of cost reduction. The BC Cancer Agency Genome Sciences Center (BCGSC or GSC) for example, is in the process of introducing $1 / 256$ reaction dilutions in their sequence pipeline, and has shown 1/1024 dilutions to work. 1/256 dilution, with median reads in excess of 800 bases, reduces the reagent cost from 
$\$ 6000$ to $\$ 22.00$ per million bases [9]. This source of cost reduction is reaching its limit however. At the GSC's current rate for customer sequencing of $\$ 4500$ per million bases, sequencing reagents represent about $35 \%$ of the total, the remainder being labour, operations costs other consumables and bioinformatics support. Even infinite dilution could only reduce that cost another $\$ 1500$. [4].

Between 1990 and 2000, improved sequencer throughput through automation and increased read lengths was a big source of cost reduction. In 1990, the ABI 370 slab gel sequencer was capable of sequencing 12,000 bases per day. By 1998, the ABI 3700 capillary machine could produce 196,000 bases per day, and by 2002 , the $3730 x \mathrm{l}$ had been pushed over 900,000 bases per day. This represents a plateau however, and subsequently, no new separation matrices or buffers have been introduced. Since 1998, improved sample purification and more selective separation matrices have allowed median production read lengths to rise from 500 to 800 bases per capillary This is still well below literature values from as early as 1998 [10], where reads up to 1300 bases per capillary were reported.

\subsection{Matrix selectivity is the next parameter to push for increased performance}

The main source of this read length plateau is in limitations of the polymer matrices used in capillaries to separate DNA. Denser matrices with better separating power tend to suffer from increased read failure in the form of loss of capillary current during the run [11]. This current decline manifests itself right after the sample is introduced into the capillary, with the current declining to less than $20 \%$ of its initial value after thirty minutes [12]. DNA bands are slowed down and arrive late at the detector, leading to the term "late starts" to describe this phenomenon. Current decline has been shown to correlate with the 
presence of large, low mobility DNA fragments in the sequencing sample which are a byproduct of the sample preparation protocol [13]. The frequency and severity of the problem varies with the type of matrix used, and it is more common with the use of less stringent, and typically less expensive, sample preparation techniques [14].

Addressing the issue of current decline is one of the few avenues of continued cost reduction available for capillary electrophoresis. While the causes of current decline had been elucidated during the development of CE separation matrices, the mechanism by which current decline occurs has never been properly explained. It is thus the objective of this thesis to understand why long DNA fragments cause current decline, and to investigate possible methods of mitigation.

In the following chapters, the background of capillary electrophoresis for DNA sequencing is developed, along with a review of previous research into the current decline problem. Two pieces of equipment for studying current decline are then described, a single capillary instrument developed specifically for this investigation, and the commercial MegaBACE 1000, a 96 capillary sequencer. Results are presented showing the causal relationship of long fragments to current decline and of current decline to reduced DNA "read length". It is then demonstrated for the first time that current decline results from a growing depletion region caused by the presence of long fragments. It is further shown that current decline only occurs if the quantity of long fragments injected is beyond a threshold level. In chapter five, a model is developed to explain the formation and growth of the ionic depletion region, and the reason for the threshold behaviour. 


\section{Chapter 2 The Principles of Capillary Electrophoresis}

The modern science of electrophoresis is generally regarded to have begun with Arne Tiselius, in the 1930's. He showed that proteins in human serum could be separated by electric current flow in a liquidfilled tube on the basis of charge and mass. As Joule heating in free solution electrophoresis causes convective diffusion leading to a loss of resolution in the separation, workers adopted liquid-permeated solid supports such as paper, starch, cellulose, acetate, agarose and polyacrylamide in slabs or sheets. The significance of narrow bore $(<100 \mu \mathrm{m})$ capillaries to control convection was first recognized by Stellan Hjertén in 1967 who introduced the concept of capillary zone electrophoresis [15]. CE development accelerated in the 1980's with the commercial availability of polyamide coated drawn glass capillaries [16]. This culminated in the extraordinarily successful development of CE for DNA sequencing in the 1990's. Today, CE is also gaining popularity as an analytical tool alongside mass spectrometry (MS) and liquid chromatography (LC), particularly for separating species of different chirality, highly polar species, and as a front end for MS separations [17].

\subsection{Principles of Electrophoresis}

The sheer number of different configurations of analytes, electrolytes, additives and detection schemes possible in electrophoresis and the interdisciplinary nature of the subject has led to an extensive and diverse literature. Sub-categories such as DNA sequencing, peptide separations and inorganic analytical chemistry approach the same phenomena with different vocabularies. 
Electrophoresis has been also influenced by the related field of chromatography, which uses a different vocabulary still. From a physics perspective, it seems easiest to begin with a mathematical description.

Electrophoresis is the process of separating charged species in a fluid by differences in electrically induced migration velocity. To perform electrophoresis, a potential difference must be set up across an ionic solution, which can be in a tube, capillary, or open box. Since many analytes are sensitive to $\mathrm{pH}$, a buffer solution is normally used, with an adequate reservoir at each electrode so that a stable $\mathrm{pH}$ is maintained throughout the run.

Through conservation of mass, it can be shown that such a system is governed by the Einstein-Smoluchowski equation for particle diffusion in the presence of a potential:

$$
\frac{\partial C_{i}(x, t)}{\partial t}=D_{i} \frac{\partial^{2} C_{i}(x, t)}{\partial x^{2}}+\frac{\partial}{\partial x}\left(z_{i} \mu_{i} E(x, t) C_{i}(x, t)\right)
$$

where $C_{i}, D_{i}, z_{i}$, and $\mu_{i}$ are respectively the concentration, diffusion constant, charge and mobility of the ith ionic species, and $E$ is the local electric field:

$$
E(x, t)=\frac{J(t)}{\sigma(x, t)} ; \quad \sigma(x, t)=\sum_{i} F\left|\mu_{i}\right| C_{i}
$$

where $J(t)$ is the current density and $\sigma(x, t)$ is the local conductivity and $F$ is Faraday's constant ( 96800 Coulomb/mol). In turn, the current is:

$$
I(t)=V\left(\int \frac{d x}{\sigma(x, t) A}\right)^{-1}
$$

where $V$ is the voltage and $L$ is the capillary's length. For a given ion in a constant field, and neglecting diffusion, each ion's average drift velocity is:

$$
v_{i}=\mu_{i} \vec{E}
$$


Electrophoresis works by exploiting the difference in mobility of different analytes. According to the Debye-Huckel theory, the surface charge of the ions or analytes is screened beyond the Debye length.

$$
\kappa^{-1}=\left(\frac{\varepsilon_{o} \varepsilon_{r} k_{b} T}{e^{2} I}\right)^{\frac{1}{2}}
$$

where $\varepsilon_{r}$ is the permittivity of the solution, $e$ is the fundamental charge and $I$ is the ionic strength of the solution. In the presence of an electric field, the analyte ion and counter ions will be pulled in opposite directions, and interact with each other hydrodynamically. Where the Debye layer is much thicker than the particle radius, $R$, the mobility is given by

$$
\mu=\frac{Q}{4 \pi \eta_{s} R}
$$

where the ion has charge $Q$ and $\eta_{s}$ is the solvent viscosity. Equation (2-6) applies to small ions and because each ion has a unique value of $Q / R$, these ions can be separated using electrophoresis in free solution. DNA, however, is much larger and has charge proportional to its length. DNA takes on a globular conformation in free solution such that internal charges are shielded by counterions that do not feel hydrodynamic effects, and the surface charge density and viscous drag thus both scale with the radius. This "free draining" property means that, to a first approximation, fragments of all lengths propagate with the same mobility given by (2-7) rather than (2-6).

$$
\mu=\frac{\rho}{4 \pi \eta_{s} \kappa}
$$

where $\rho$ is the DNA globule's surface charge density [18]. DNA must therefore be separated in a viscous medium which can interact with the DNA polymer and produce a length-dependent drag, preventing this 
free draining behaviour and breaking the length scaling degeneracy between driving force and drag.

\subsection{DNA Propagation in Matrices}

There are several regimes for DNA molecule propagation in polymer matrices; these are shown in Figure 1, reprinted from [19]. For polyacrylamide matrices, it is thought that the equilibrium reptation regime $(C)$ holds for DNA strands up to several hundred bases in length [20]. There is evidence that longer fragments may propagate in the regime of oriented reptation as, at high driving fields, electropherograms are concluded with a large peak followed by no signal, suggesting all the larger peaks are propagating at the same rate. Though reptation regimes and the governing equations are typically the central point of interest in electrophoresis, much of the work presented here hinges instead on properties of ions and the background electrolyte, and therefore additional background will be provided in this area. 


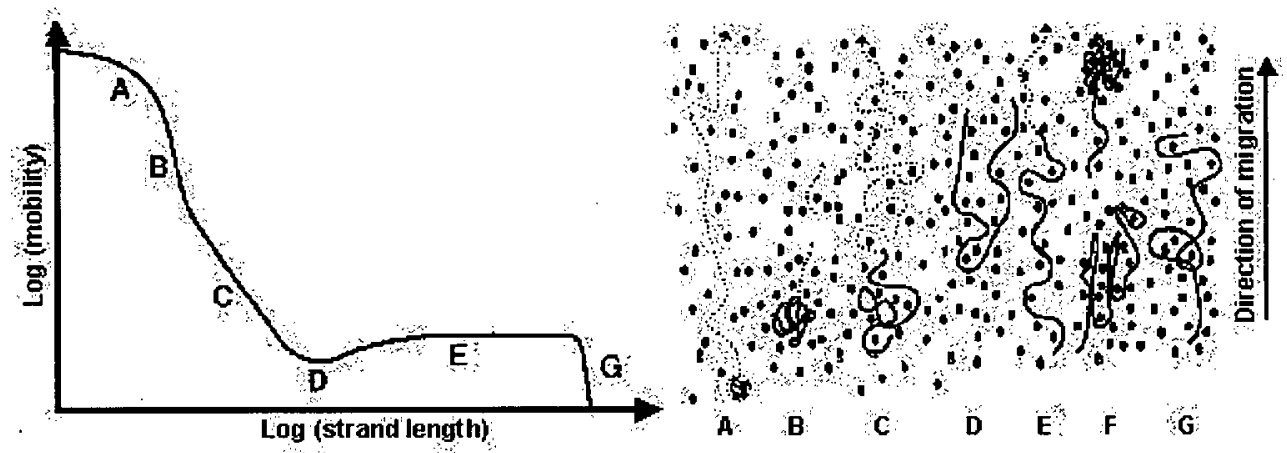

Figure 1 Schematic description of electrophoretic properties of DNA, reprinted from [19]. [A] Ogston regime - DNA stays coiled and is sieved. [B] Entropic Trapping: The random coil jumps between larger pores. [C] Near equilibrium reptation: The random coil migrates head first through the matrix. [D] Reptation trapping: The DNA is slowed by both ends pulling though the matrix and forming U-shapes. [E] Oriented Reptation: The DNA uncoils and goes through the matrix with one end leading. [F] Geometration: High fields may be characterized by hernias and "bunching" instabilities. [G] Very long molecules may not migrate through matrices at all, possibly because they form knots around gel fibers.

\subsection{Other Properties of Electrophoresis}

Amongst the properties of electrophoretic systems which must be taken into account is the relationship of temperature and solution conductivity. The viscosity of aqueous solutions varies according to $\eta \sim \exp \left(E_{a} / k T\right)$, where $E_{a}$ is the activation energy for the viscous flow [21]. This means that ion mobility and therefore buffer conductivity, is dependent on temperature. A widely employed rule of thumb is that between $20-60^{\circ} \mathrm{C}$ the conductivity changes by $2 \% /{ }^{\circ} \mathrm{C}$. This is true in the present case, and experimental data is presented in Appendix D 
which shows this rule to be quite accurate. Temperature acts as a limiting factor in the separation speed as the temperature gradient between the center and wall of the capillary caused by Joule heating will result in a parabolic conductivity profile which contributes to band broadening [22].

A feature of all capillary electrophoresis is electroosmotic flow (EOF). Under aqueous conditions glass possesses an excess of negative charge. This results in a net positive charge along the surface of the aqueous region. When an electric field is applied, the mobile counterions to this surface charge drift with the fields, but there is no equivalent motion of the fixed surface charges. Consequently, bulk fluid flow is established towards the cathode (negative electrode). EOF differs from hydrostatic flow in that flow is generated within the charged region next to the capillary wall, so the radial velocity profile is flat, and analytes are not dispersed. The EOF velocity is nominally

$$
v=\frac{\varepsilon \varsigma}{\eta_{s}} E
$$

where $\varepsilon$ is the permittivity of the solution, and $\zeta$ is the electric potential at the surface where shear occurs, commonly called the zeta potential. In a real capillary, (2-8) is potentially misleading as it assumes that the EOF driving force is constant throughout the capillary [23]. Different local conditions and defects in the capillary walls could cause different local driving forces for EOF, potentially leading to non-flat flow profiles and analyte dispersion. EOF is also highly dependent on solution $\mathrm{pH}$, particularly in fused silica capillaries and can rise by an order of magnitude with $\mathrm{pH}$ increase from 2 to 12 . At $\mathrm{pH} 8$ where DNA sequencing takes place, the EOF "mobility" $(\varepsilon \zeta / \eta)$ in an uncoated capillary is on the order of $60 \times 10^{-9} \mathrm{~m}^{2} / \mathrm{Ns}$ [24], much higher than, and in the opposite direction to mobility of DNA sequencing fragments which range from 3 to $20 \times 10^{-9} \mathrm{~m}^{2} \mathrm{Ns}$. EOF must therefore be suppressed for 
DNA sequencing, using capillary surface treatment or self-coating matrices. Suppression of EOF also prevents dispersion from inhomogeneities in the EOF mobility [25]. In modes of CE where a more acidic buffer can be used, EOF can be used to advantage: increasing the effective length of the capillary or allowing positive and negative analytes to be separated together. The latter is done by hydrostatically introducing the sample plug and then conducting an electrophoretic separation while the entire contents of the capillary are flowing past the detector under the influence of EOF. EOF is in fact an integral part of almost all types of CE except for DNA sequencing.

\subsection{Electrokinetic Injection and Sample Stacking}

In sequencing applications, DNA is injected into the capillary by electrophoresis rather than hydrostatic pressure as in some other forms of CE. A well containing the sample is placed at the cathode end of the capillary and the electric field is applied. The sample well is then removed and replaced with running buffer so the run can proceed. This "electrokinetic injection" enables sample compression, also known as stacking, which is crucial to the success of capillary sequencing as resolution is inversely proportional to injection peak width [26].

Sample compression affects both the concentration and width of the injected sample slug. The free solution mobility of DNA fragments is $37 \times 10^{-9} \mathrm{~m}^{2} \mathrm{Ns}$ [27], whereas in the matrix it drops to between 20 and $3 \times 10^{-9} \mathrm{~m}^{2} \mathrm{Ns}$ for fragments from $30 \mathrm{bp}$ to $1000 \mathrm{bp}$. This causes an increase in concentration from $C_{\text {sample }}$ to $C_{\text {capillary }}$ by the amount

$$
C_{\text {capillary }}=C_{\text {sample }} \frac{\sigma_{\text {bge }}}{\sigma_{\text {sample }}} \frac{37 \times 10^{-9}}{\mu_{D N A}}
$$

where the sample conductivity $\sigma_{\text {sample }}$ of sequencing fragments is typically one hundred times lower than the background electrolyte conductivity in the matrix $\sigma_{\text {bge }}$. This does not however compress the 
bands in time, so that if electrokinetic injection is done for ten seconds, one would expect the resulting DNA bands to take ten seconds to cross the detector, plus the time attributable to diffusional broadening. In fact, peak width compression of up to a factor ten has been observed [28] immediately after injection, and a factor of three compression was consistently observed in our work with the MegaBACE sequencer, using standard sample purification procedures and resuspension of the sample in $\mathrm{dH}_{2} \mathrm{O}$.

Temporal sample stacking requires that the high field/low field boundary initially present at the capillary entrance move with the DNA so that DNA arriving later in the injection slows down later, and therefore closer to the leading edge of the injection peak. While it has been shown experimentally that lower sample salt concentration promotes stacking [13], and that adding fast-moving $\mathrm{OH}^{-}$ions after injection can also compress the sample [29], no mechanism by which the boundary moves has been hitherto proposed.

Of interest to the present discussion is the amount of DNA injected into the capillary. While attempts were made at direct measurement of the DNA injected into the capillary via spectrophotometric measurement of the eluted sample, the quantities involved were too low to give meaningful results. The mass of injected fragments in the following experiments was therefore calculated according to:

$$
M_{i n j}(t)=\frac{\mu_{D N A} C_{D N A} M W_{D N A}}{\sigma_{\text {sample }}} \int_{0} I(\tau) d \tau
$$

where $\sigma_{\text {sample }}$ is the sample conductivity (Sieverts ${ }^{*} m^{-1}$ or $\left.(\Omega m)^{-1}\right), C_{D N A}$ in $\left(\mathrm{mol}^{*} \mathrm{~m}^{-3}\right)$ is the DNA concentration in the sample, MWDA 295 $\mathrm{g} / \mathrm{mol}$ for one nucleotide, and $I(t)$ is the capillary current (Amps), integrated over the injection time $t$. The mass of injected DNA is proportional to its fraction of all the charge carriers, so injection from 
lower conductivity electrolytes results in larger quantities of injected DNA. Note that in this scheme DNA is treated as a group of individual nucleotides: the lengths of the actual fragments are unimportant in this calculation.

Two related formulas are also relevant here. The integral in $(2-10)$ is actually the total charge flowing into the capillary during injection. This can be extended to the total loaded charge over the run

$$
Q\left(t_{r}\right)=\int_{0}^{t_{r}} I(\tau) d \tau
$$

where $t_{r}$ is the electrophoresis run time. In order to characterize changes in current, we similarly define the normalized total loaded charge as:

$$
N\left(t_{r}\right)=\frac{\int_{0}^{t_{r}} I(\tau) d \tau}{I(0) t_{r}}
$$

where $I(0)$ is the current at the start of the run.

\subsection{Capillary Sequencing for DNA}

The details of DNA properties, sample preparation and sequencing protocols are contained in Appendix A. Here we suffice to say that DNA sequencing requires creating a quantity of DNA fragments of a common starting point in the sequence and monotonically increasing length from 20 bases to 1000 bases such that there is enough DNA of each length to be detected (typically $10^{8}$ molecules per size band). Each fragment terminates with an $A, C, G$, or $T$ nucleotide that is labeled with one of four different fluorescent dyes, so sequencing involves separation by size and detection of the end-label emission wavelength. This method, developed by Sanger, 
Gilbert and Maxam in 1977 [30, 31], initially used four separate lanes on a polyacrylamide slab gel with radio-labeled fragments for detection. Hood et. al. improved this with four-color florescent labeling [32] in 1986, enabling sequencing in one lane per sample. This led to the first generation of semi-automated slab-gel sequencers, such as the Applied Biosystems 370 family. Despite read lengths of over 600 bases per lane, these machines were limited by manual intervention in gel casting, and were limited to low field strengths because of excessive joule heating. Inhomogeneities in the gel also caused lane alignment problems, limiting lane densities and making automated base calling difficult.

Capillary electrophoresis for DNA sequencing was introduced in $1990[33][34][35,36]$, although technical obstacles delayed the appearance of commercially available array sequencers until 1997. The principal motivation was a potential increase in separation speed of an order of magnitude through improved heat dissipation, and superior lane tracking. A less obvious advantage is the design flexibility allowed by a discrete capillary array. The cathode end can be arranged to match standard 96 or 384-well reaction plates while the anode and detector regions can have any physical layout the designer desires. Samples can be injected directly from 96 or 384-well reaction plates and matrix replacement can be performed by hydrostatic pumping from the anode. It is also possible to replace sample, matrix and buffer containers so only the capillaries themselves have to be cleaned between runs. This continues to be a notable advantage over lab-on-a-chip electrophoresis systems, where the capillaries and buffer wells are all in one monolithic structure. 


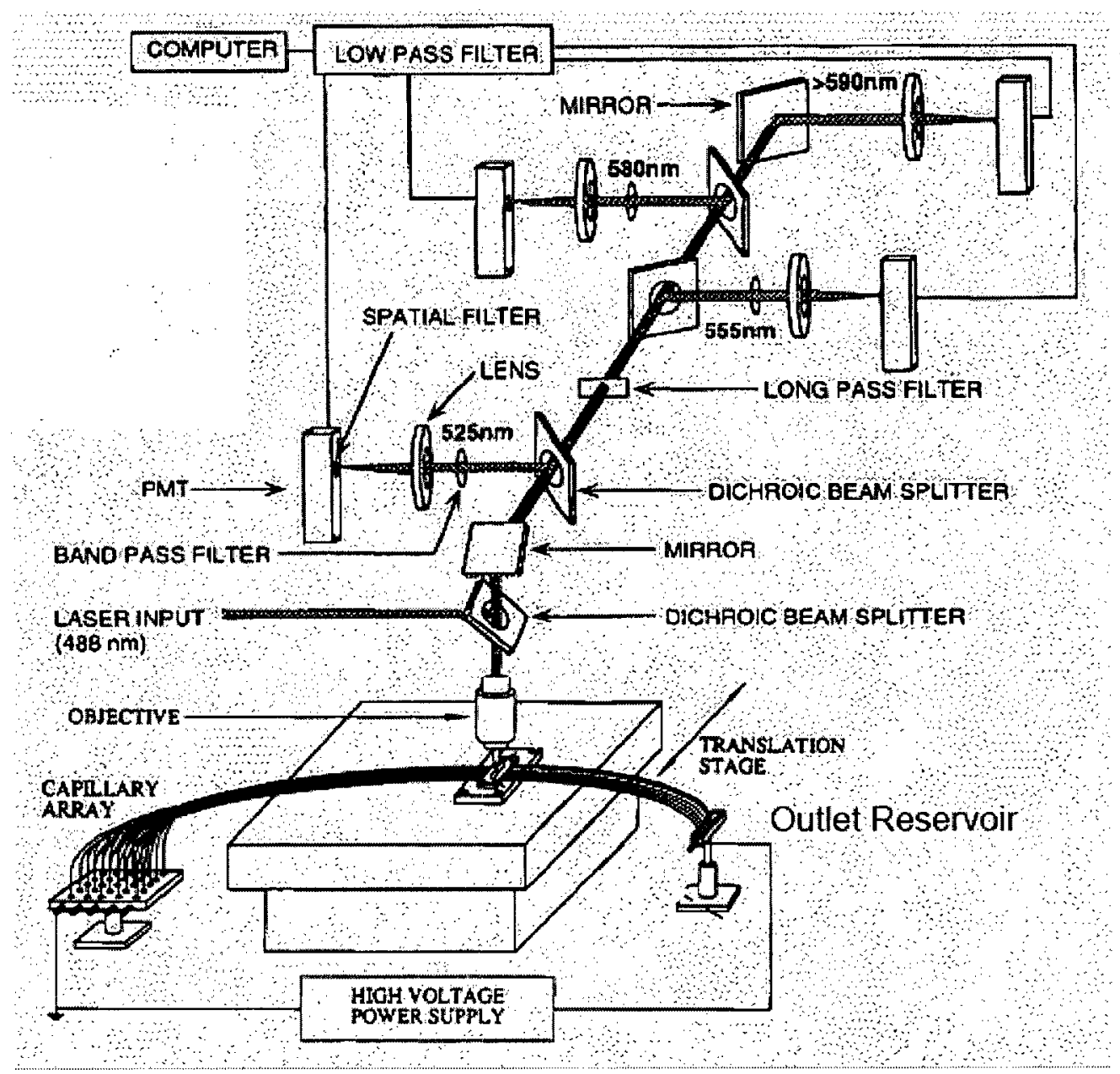

Figure 2 The original confocal array developed by the Mathies group. Reprinted from [37]

The biggest technical challenges in CE sequencing were multicapillary fluorescence detection and development of suitable separation matrices. To address the former, Mathies et. al. developed a scanning confocal microscope system [37] [38], shown in Figure 2, which led to the introduction of the MegaBACE 96 channel sequencer in 1997. This system scanned a microscope objective across the capillaries which were lined up side by side, while the rest of the optics remain fixed. Although it had to scan 96 capillaries every 1.7 seconds, it could devote full laser power to each capillary. This approach was 
later scaled to 384 capillaries in the MegaBACE 4000 . Dovichi and others [39] concurrently developed a sheath flow approach where upon exiting the capillary, fragments were imaged as they were hydrodynamically focused by matrix flowing past the outlet. The laser was aimed down the line of eluting samples from one end, and the resulting fluorescent spots were diffracted off a grating and onto a CCD array. Different colors produced different positions on the CCD. This system gained flexibility with fluorophores at the expense of reagent consumption and signal strength. While it was proven successful on the 3700 , the $A B I 3730$ that followed, imaged its capillaries from one end directly through the capillary glass. This uses only a thirtieth as much buffer and matrix [9], and provides improved uniformity in the fluorescence signal from one capillary to another.

The biggest obstacle to developing viable capillary array sequencers was in finding a separation matrix that was selective, electrically stable, replaceable and resistant to EOF. Cross linked polyacrylamide matrices which had worked well in the slab format and could be covalently bonded to the capillary wall to prevent EOF were initially employed. Both sample-related and sample-independent matrix instability $[33,35,39-41]$ was reported however. Moderate sample-independent current decline was observed that could be periodically reset by clipping a small portion of the cathode end of the capillary. Bubbles were also observed to form spontaneously near the cathode end of the capillary, particularly at fields over $300 \mathrm{~V} / \mathrm{cm}$. This work was well reviewed and expanded by Swerdlow et al. in 1992 [42]. They found that sample-independent current decline stemmed from the formation of a resistive region at the cathode end of the capillary, thought to be due to a change in the relative conductivity of the ions across the matrix/buffer boundary (this is known as the transference number). Figeys et al. likewise used capillary cutting to reveal the axial 
conductivity profile after running for up to 280 minutes [43]. Their results appear to be consistent with a change in relative conductivity of the different ionic species across the matrix/buffer interface coupled with diffusion of the depletion region into the capillary. Changing buffer constituents, particularly adding unpolymerized matrix monomers to the buffer ameliorated these effects [44]. The mechanism put forth by Swerdlow et al. [42] for this current stabilization is that the monomers equalize the relative mobility (transference number) of the ionic species across the gel/buffer boundary. Spontaneous bubble formation likewise seems to have been largely mitigated by the switch to non-crosslinked matrices. Figeys [44] did not report bubble formation with such matrices, even with runs of hundreds of minutes at $300 \mathrm{~V} / \mathrm{cm}$ and notwithstanding the high fields associated with the depletion region.

The MegaBACE adopted linear polyacrylamide (LPA 2-4\% w/v) [45-47] with average molecular weights of $\sim 10^{6} \mathrm{Da}$. EOF is suppressed using a surface treatment [48] [49] which suppresses surface charge and binds the linear polyacrylamide to the capillary wall. $A B I$ introduced its proprietary Performance Optimized Polymer (POP) line of matrices for the 3700 , based on poly(dimethylacrylamide) PDMA [50]. The PDMA polymer suppresses EOF though a moderate degree of hydrophobicity. This allows the polymer to bind to the capillary wall but keep much of its length off the wall, leading to a socalled "loopy" conformation [51]. PMDA based matrices, therefore, allow $A B I$ to use non-coated capillaries. Both systems used Tris/TAPS as a running buffer because it was found to produce better quality separations than Tris Borate, the traditional slab-gel buffer. It is thought that the latter may be prone to formations of complexes with residual glycerol from cycle sequencing reactions [45]. Tris and TAPS, respectively a large organic acid and base, in equal concentration only 
dissociate about $50 \%$ each so the solution also has a comparatively high buffering capacity [52]. The exact content of the commercial matrices is not known, but it is clear that sample independent current stability has been attained through judicious selection of buffer and matrix components. Current decline from large fragments, however, continues to be a concern [53] [12] for both LPA and PDMA based matrices.

The introduction of the MegaBACE and 3700, combined with efforts to automate upstream sample preparation resulted in a dramatic increase in the pace of sequencing for the human genome project. $A$ big factor may have been Celera Genomics' 1998 entry into the "race" to sequence the human genome using 300 of the new 3700 s. The scientific significance of Celera's contribution remains the subject of debate $[54,55]$, but it is clear that it stimulated the uptake of large numbers of sequencing machines by all the major genome centers. From the beginning of the HGP in 1990 , to 1998 , about $3 \%$ of the genome had been sequenced[5,56], while from 1998 to 2001, the other $97 \%$ was completed.

It is easy to understand how, in the context of the time, both sequencers were introduced to market in haste. The 3700 experienced up to $30 \%$ downtime for various mechanical maladies and the sheath flow detector system consumed large quantities of polymer. The MegaBACE was somewhat more reliable but required considerable operator involvement for plate exchanges. Under ideal circumstances, however, the MegaBACE was capable of read lengths of up to $800 \mathrm{bp}$ in 1998 , whereas the 3700 could only manage up to $600 \mathrm{bp}$ [9]. It is reasonable to suppose that with development of dilute reaction protocols, the sensitive detector and low matrix usage of the MegaBACE might have made it the sequencer of choice early on, and allowed the manufacturer to develop a next generation machine with 
better automation. In the end however, the sequencing community largely rejected the MegaBACE for one significant reason. Under real conditions, using DNA samples prepared using $E$. coli growth protocols, the MegaBACE had up to a $20 \%$ capillary failure rate. These failures were characterized by declining capillary current and the delayed onset of peaks at the detector. While the 3700 also displayed the same failure mode [53], the rates were considerably lower Each read failure represents not only a loss of sequencing time, but a loss of the very expensive cycle sequence reaction, as well as all the upstream sample preparation steps. Combined, these failures represented at least $10 \%$ of the cost of sequencing at the time, so the cost of read failure to the human genome project was perhaps as high as three hundred million dollars. This is in addition to the fact that using PDMA based matrix to mitigate the problem forced workers to live with lower average read lengths. While the $A B I$ machines always had superior automation, it is likely that the issue of run failure due to current decline decisively cost the MegaBACE its initial lead in sequencing. The result is that today, virtually all major genome centers only use $A B I$ sequencers.

\subsection{Current Decline and Mitigation Efforts}

Swerdlow et al.[42] studied the effect of DNA sequencing sample characteristics on current stability, and explicitly showed that rapid current decline was associated with the injection of a sufficient quantity of fragments longer than $1300 \mathrm{bp}$, and that smaller quantities did not cause the effect to occur. After Swerdlow, further discussion of current decline was limited, although it was addressed in the important pair of papers from the Karger lab which point out that, "Indeed the problem of sample cleanup for the successful operation of CE has not been sufficiently emphasized" [14]. Karger's lab developed a 
purification method based on centrifugation through filters, which, while effective, proved too expensive for sequencing centers to consider implementing. In the second paper, they reported that quantities of $8 \mathrm{~kb}$ M13 DNA added back to sequencing samples after filtration and then de-salted did cause current decline. They also demonstrated that adding salt back to de-salted but template laden sample would prevent the current decline, albeit at the cost of reduced loading and separation efficiency. Other techniques found to prevent current decline include multiple sample injections [53] and resuspending reaction products in template suppression reagent (TSR) such as dilute agarose $[57,58]$. While filtering reduces the amount of template without reducing the quantity of short sequencing fragments, adding salt or multiple sample injections is likely to reduce the total quantity of DNA injected, including the template, below the threshold for current decline. Results are shown Appendix B which demonstrate that using Agarose as TSR reduces the total quantity of DNA injected, by reducing the injected template below the threshold for current decline.

All these methods have drawbacks however. More stringent cleanup adds cost. Increased ionic strength in the sample reduces the total amount of DNA loaded which may result in inadequate signal strength as processes move toward smaller volumes and lower quantities of sequencing fragments. TSR may further reduce the quantity of loaded sequencing fragments, and also presents viscosityrelated liquid handling issues.

At present, current decline in the $A B I$ sequencers is controlled through a combination of more dilute reactions with less starting DNA, and use of the fault tolerant POP-7 matrix (believed to be a combination of PDMA and LPA which is still self-coating). In the future, more selective matrices will evolve from a better understanding 
of the current decline problem; it is hoped that the present work will contribute to that understanding. 


\section{Chapter 3 Apparatus and Methods to Investigate Current Decline}

Initial experiments on current decline and its relationship to read length degradation were carried out on MegaBACE DNA sequencers located at the BC Cancer Agency Genome Sciences Center. While the MegaBACEs generated 96 channels of current and fluorescence signal data at once, it was not possible to observe other characteristics of the capillaries while the machine was operating. A custom single capillary instrument was therefore constructed, mimicking the MegaBACE as much as possible by using the same capillaries, separation matrix and buffer. This instrument was configured with the detection instrumentation at the cathode end of the capillary, rather than the anode end as in conventional sequencers, as it was known from the literature that the source of current decline was likely to be at or near the cathode.

Experiments with the single capillary instrument revealed considerable variability in the injection quantity of highly purified samples. The reason for this variability is unknown, but it made it impossible to get reproducible current decline behaviour with purified samples. It was concluded therefore that extensive experiments on a capillary array machine were also required, so a MegaBACE was procured and installed at UBC. In this chapter the MegaBACE, the custom-built single capillary instrument; and associated methods are described.

All capillary separation devices operate on the same physical principles. A capillary must be positioned between two baths of 
electrolyte, which should be level with each other to prevent siphoning. There must be a high voltage power supply connected to electrodes in each bath so that current can pass through the capillary. There must also be some sort of analyte detection scheme, be it through the capillary wall or as the analytes elute from the end of the capillary. The detector needs to be far enough from the cathode to allow enough separation, and near to the anode so that the capillary isn't unnecessarily long. There must also be some reasonably straightforward way of replacing the anode and cathode wells to switch between sample, buffer and matrix, and some system for pumping the matrix and rinse water through the capillary. Finally, there must be a system for heating and stabilizing the capillary temperature so as to prevent secondary structure formation in the DNA or thermal band broadening [22]. In light of the foregoing, it is not surprising that commercial sequencers share many similarities. The differences between machines are mostly in the degree of automation, and layout issues related to the number of capillaries in the machine. 


\subsection{The MegaBACE}

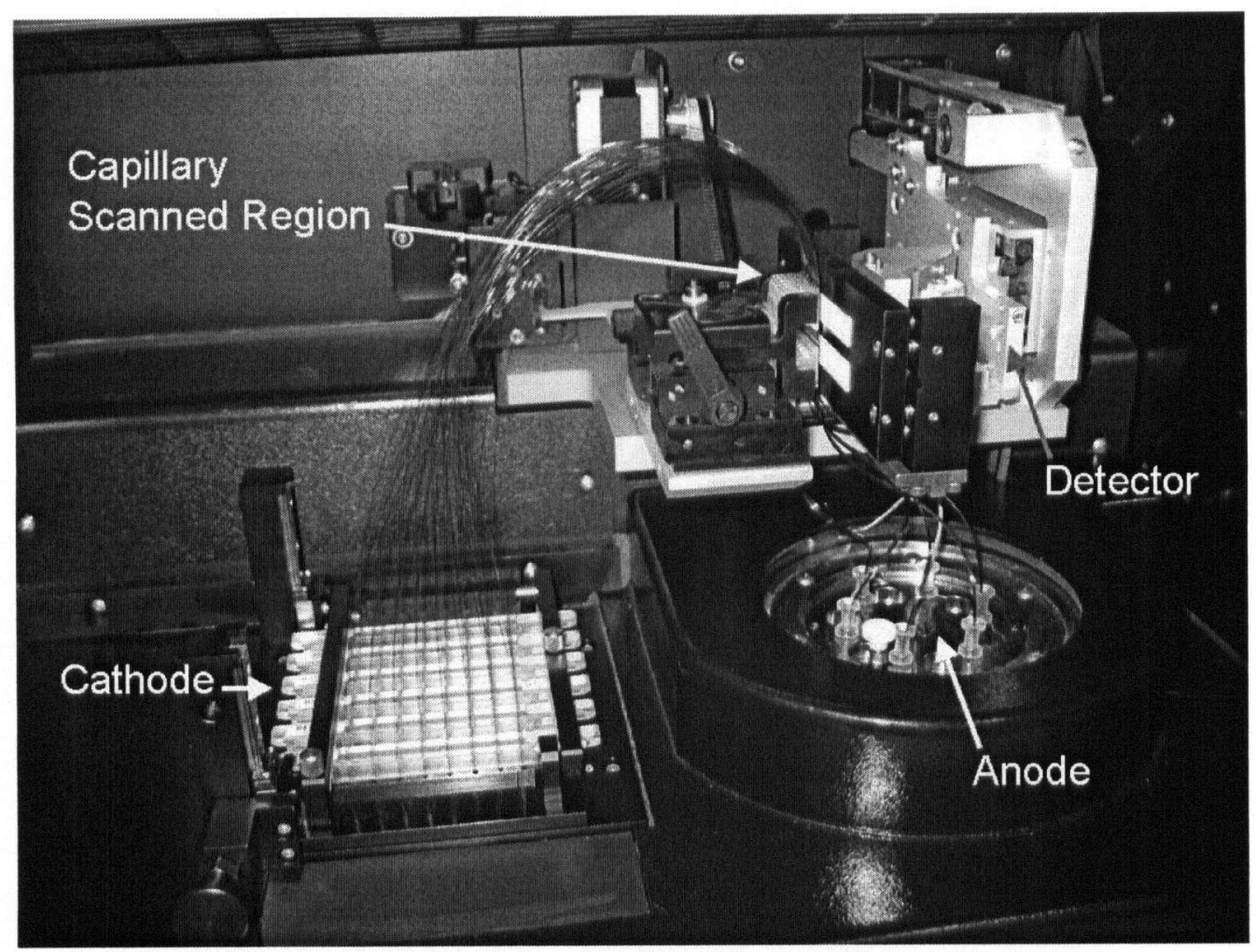

Figure 3 The airbox in the MegaBACE showing the capillary array, anode pressure vessel and cathode array

The MegaBACE 1000 is the first generation 96 channel DNA sequencer from Molecular Dynamics (Subsequently Amersham Biosciences and now part of General Electric Healthcare). Figure 3 shows the interior of the MegaBACE's air box, which is kept closed throughout operation. Use of an air box for heating is more or less mandated by the use of capillary arrays, and ABI's current 3730 machine still uses one, albeit smaller than the MegaBACE's. On the left, six bundles of sixteen capillaries are arrayed to match the 96 well plates which are positioned below the air box. A platinum electrode hangs next to each capillary. The electrodes are grounded across $100 \mathrm{k} \Omega$ resistors and the resulting voltage drop is used to measure 
current. The capillaries are OD $225 \mu \mathrm{m}$, ID $75 \mu \mathrm{m}$ and $60 \mathrm{~cm}$ long. They are coated with a polyamide resin rendering them highly resistant to breakage. A detector window is etched into the coating $40 \mathrm{~cm}$ from the cathode end, of each capillary, and each array of sixteen detector windows is held together with a plastic clip.

All six of the detector window arrays are brought together in a linear array to be scanned by the confocal microscope. The microscope's objective scans the array at $\sim 1.7$ scans/sec. Four colour flourescent detection is achieved in the following manner: Flourescent light is routed back through a dichroic mirror to separate it from the laser light, then through another dichroic mirror which divides the four flourescent signals into two pairs of lower and higher frequency. Each beam passes through a scanner which switches between two final filters in phase with the objective scan. Two channels are detected on the outbound scan and two on the inbound scan. In this way the four fluorescent wavelengths can be detected using only two photomultiplier tubes. Note that this is different from the original Mathies' design shown in Figure 2, page 15, where the light is sequentially separated into four channels.

At the lower right in Figure 3 is the anode, where each bundle comes together and enters the high pressure chamber from where matrix is pumped from six $1 \mathrm{ml}$ microcenterfuge tubes. The entire chamber is at high voltage during operation. The LPA matrix used in the MegaBACE is quite viscous, so the MegaBACE operates with 1000 psi of matrix injection pressure. One modification that the GSC made to the standard sequencing protocol was to replace the microcenterfuge tubes of LPA with running buffer at the anode after injection of the LPA into the capillaries but before sample injection. This was done to save money as tubes of LPA uncontaminated with DNA could be used for three runs rather than one, saving $\$ 40.00$ per 
96 well plate. This produced a significant effect however: upon replacing the LPA vial with buffer, and without injecting DNA, the capillary current would decline by about $50 \%$ over fifteen minutes and remain constant at that value. No equivalent relationship between buffer contents and capillary current was observed at the cathode. This effect, which did not affect sequencing performance, is discussed further in Appendix C.2.

\subsection{The Single Capillary Instrument}

\subsubsection{Mechanical Design}

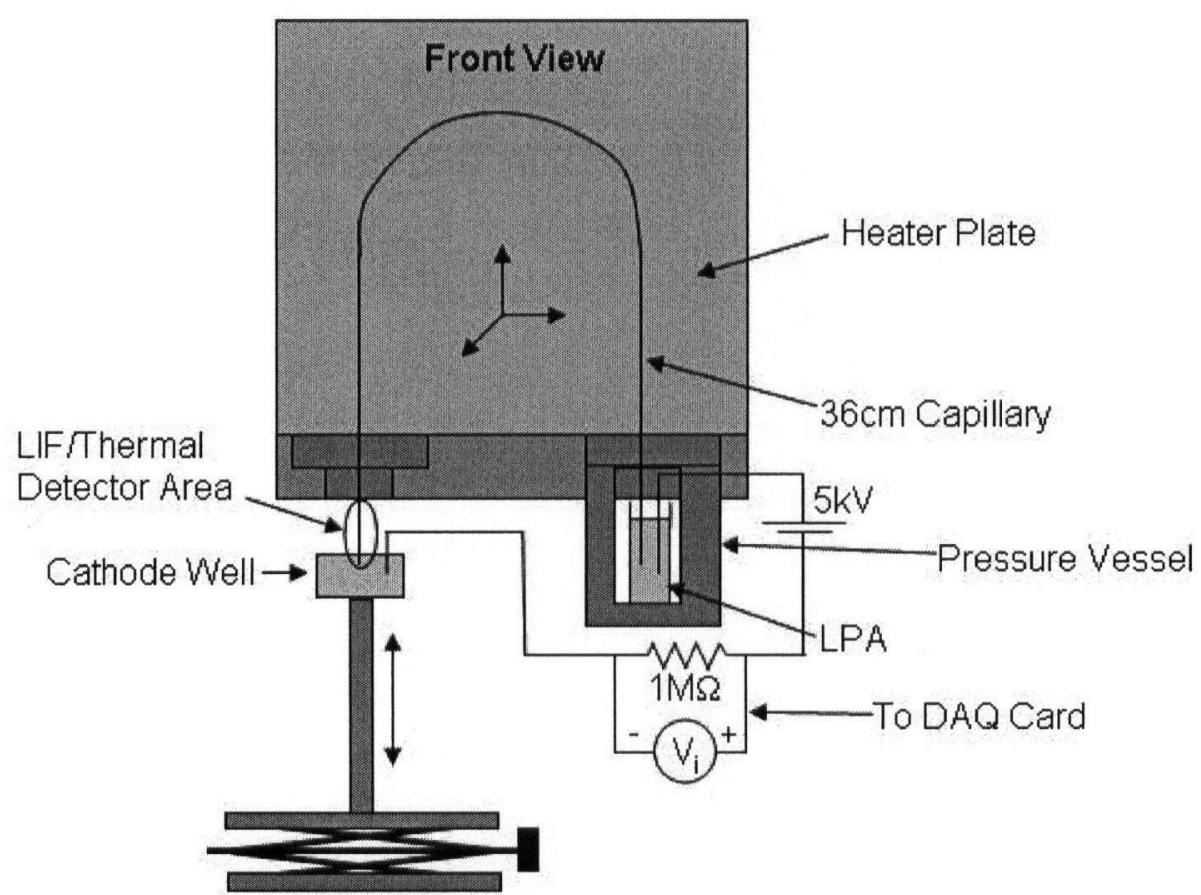

Figure $4 \mathrm{~A}$ front view schematic of the single capillary sequencer. Note the position of the laser Induced fluorescence detector close to the cathode, rather than the anode on the MegaBACE.

The single capillary instrument we developed is schematically depicted in Figure 4. The layout of the machine was influenced by the 
need for clearance below the capillary's ends, and a desire to keep the detector optics compact and on one level. The solution was to mount the CE apparatus and detector assembly on a small optical breadboard on $15 \mathrm{~cm}$ posts above the main breadboard. This gave enough room under the capillary to position the cathode sample/buffer holder using a lab jack.

This layout is essentially the same as the MegaBACE, which has the whole optical detector assembly midway up the machine, behind the capillary air box which is above the pneumatically driven anode and cathode exchange mechanisms. In order to focus and align the capillary vertically and horizontally, the entire capillary assembly, anode and cathode caps, main deck and heater plate were mounted on a three-axis stage, while the detector optics were fixed.

This design sought to limit the unheated or uninsulated portions of the capillary as much as possible to prevent bubble formation through matrix shrinkage. $26 \mathrm{~cm}$ of the $36 \mathrm{~cm}$ capillary was taped to an aluminum plate heated on the other side by a kapton heater (Omega). The $5 \mathrm{~cm}$ of capillary at the anode was inside a pressure vessel and insulated from temperature changes and only $5 \mathrm{~cm}$ at the cathode was exposed to room temperature. This arrangement was stable enough that matrix shrinkage-induced bubbles could be avoided if sample and buffer wells were changed in a timely manner.

The pressure vessel containing the anode consists of a stainless steel cap into which screws a cylinder containing a microcenterfuge tube which contains the desired reagent: polymer matrix, buffer or rinse water. The cap is plumbed for the high pressure and voltage input lines, the platinum anode electrode, and the capillary pressure fitting. The two sections are screwed together (13tpi, 1.25" diameter, factor of safety under maximum pressure is 100) and are sealed with an O-ring. An Upchurch $F^{\star} 150$ steel compression fitting 
surrounding a $1 / 16$ " OD, $250 \mu \mathrm{m}$ ID PEEK tube is used to seal to the capillary. The fitting compresses a ferrule into the PEEK tubing which in turn squeezes the close-fitting capillary. The high pressure input line, also PEEK tubing, is attached to the chamber cap in the same way. The steel cap is secured with four nylon screws to the main deck of the CE instrument which is made from acrylic. The lower half of the pressure chamber is also held in an acrylic cylinder so when the system is assembled, only the capillary's steel pressure fitting and a part of the surrounding cap are exposed at high voltage. Voltage was limited by the proximity of the top of the pressure fitting to the heater plate which caused electrical discharges above $5000 \mathrm{~V}$, the result of a compromise between minimizing the exposed region of capillary and proximity of the cap to the heater plate. A maximum voltage of $5000 \mathrm{~V}$ was adequate for these experiments.

High pressure was delivered to the anode pressure vessel from a nitrogen cylinder with a high pressure regulator through stainless steel tubing to an exhaust valve and then a high pressure quick connect fitting. A length of 5000 psi PEEK tubing connected the latter to the anode pressure vessel cap. In principle, PEEK tubing could be used right from the regulator, but integrating the three-way exhaust valve to vent the system after filling the capillary was most elegantly accomplished with fixed plumbing. The quick release fitting proved valuable in that other devices could be attached to the pressure line in order, for example, to flush complete MegaBACE capillary bundles offline. The LPA polymer can be pumped into the capillary at a pressure as low as $300 \mathrm{psi}$, but in order to clear occasional blockages which can form if the capillary is allowed to dry out with polymer inside, it was convenient to have higher pressure available. This instrument could apply up to $1500 \mathrm{psi}$ to the anode pressure chamber. 
The cathode end of the capillary ran though the same stainless steel cap and ferrule assembly as the anode, originally built to test the hypothesis that bubbles might re-dissolve by pressurizing the whole capillary. While bubbles ultimately proved to be of secondary importance, the ferrule clamp held the capillary in very good alignment with the detector. Samples and buffers were contained in wells drilled in a rotating acrylic disk mounted on a lab jack. The sample holder had $200 \mu \mathrm{L}$ buffer wells and $25 \mu \mathrm{L}$ sample wells.

The electric field was supplied by a high voltage Spellman power supply connected to the anode pressure vessel directly. The anode electrode was a length of platinum soldered to the pressure cap and hanging close to the capillary. The platinum cathode electrode was connected to the Spellman's ground through a $1 \mathrm{M} \Omega$ resistor. The voltage was measured across that resistor to find the capillary current. Both this signal and the PMT output were acquired by the computer's Labview software though an National Instruments-6025E A/D card. 


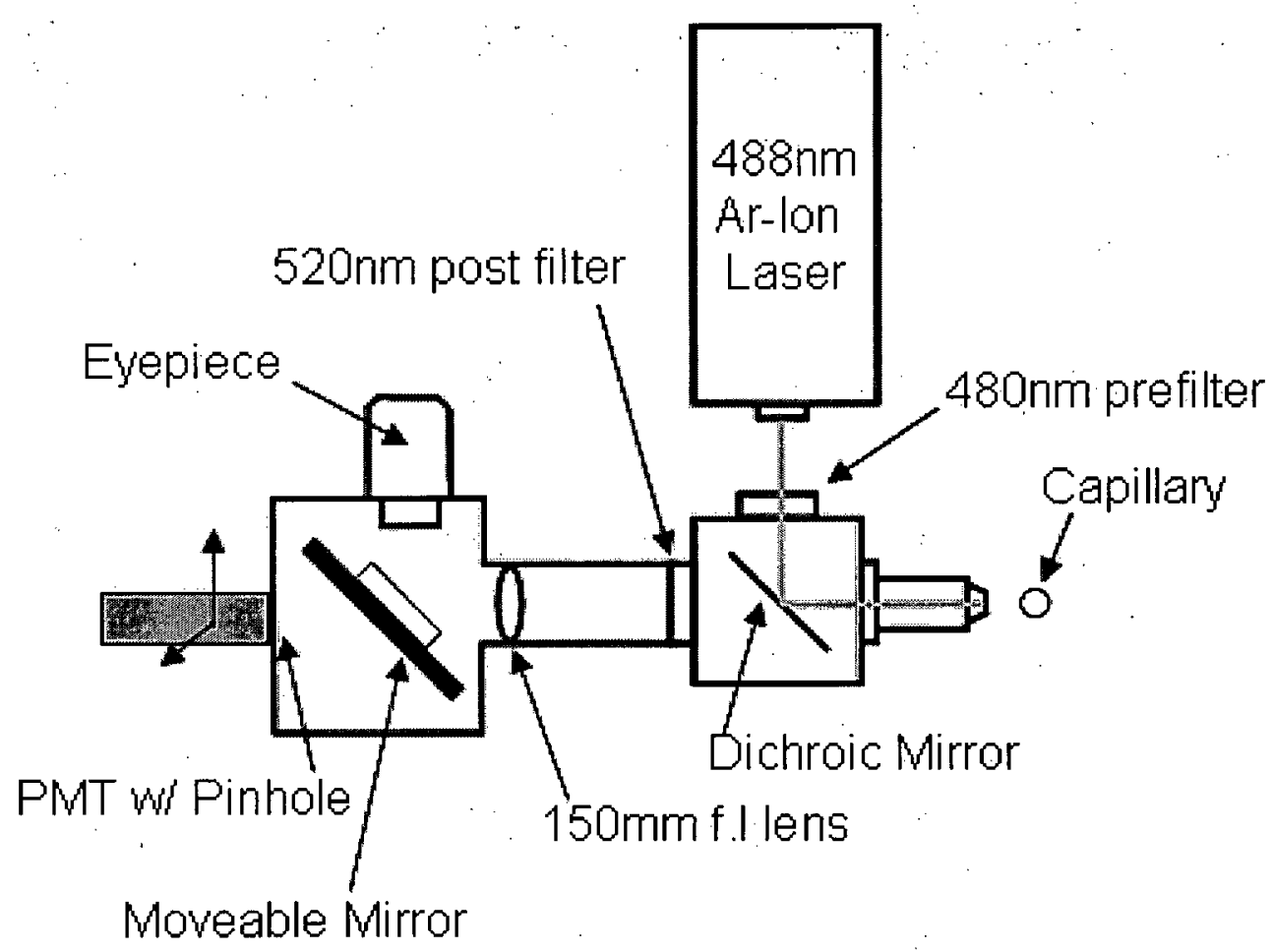

Figure 5. A schematic of the confocal fluorescence detector system. In the actual system, the laser was mounted below the apparatus and the input beam arrived with a series of mirrors.

\subsubsection{Fluorescence Detection}

A schematic of the laser induced fluorescence (LIF) detector is shown in Figure 5. It is based on the design of Mathies et al. which was used in the MegaBACE [59]. In this case there is only one fluorescent dye colour, rather than four, and therefore only one filter set and detector. The principle of confocal laser induced fluorescence microscopy is to focus the laser beam on the capillary, and then form an image of the resulting fluorescent signal by sending the output of the microscope objective through a dichroic mirror and on to the detector. The dichroic mirror, pre and post filters, are chosen to match 
the characteristics of the chosen fluorophor, so the laser light is reflected and fluorescent light transmitted. The present work was done using Sybr Green intercalating dye (Molecular Probes), which is excited near $488 \mathrm{~nm}$ and emits at $520 \mathrm{~nm}$, and a Sybr Green filter set (Chroma Inc.). The microscope objective was 10X (Roylin Optics). After passing through the $520 \mathrm{~nm}$ post-filter the beam passed through a biconvex lens with a focal length of $150 \mathrm{~mm}$ which formed a real image on a pinhole plate in front of the photomultiplier tube (PMT). The pinhole could then be moved in the image plane via a small $X-Y$ stage so that the fluorescent spot induced by the laser fell exactly on the pinhole. A moveable mirror was also mounted in the light box which could be interposed in the beam after the lens, so as to redirect it upward where it was confocal with a $26 \mathrm{~mm}$ Plössl eyepiece. This feature proved invaluable in aligning the capillary at the laser beam focus before doing a run. The mirror itself moved on a linear stage actuated by a pneumatic cylinder connected to a manual five-way valve outside the light-box. Optical detection was done with a Hamamatsu $\mathrm{HC}-120$ photomultiplier tube. It was driven through a variable gain circuit as per Hamamatsu's schematic, and the output was filtered at $1 \mathrm{kHz}$, before going to the $A / D$ card.

Initial alignment of the optics required aligning the pinhole so that it was confocal with the fluorescent spot from the objective. Coarse alignment was done by removing the cover of the light box and illuminating the capillary with a bright light so as to make the capillary image visible to the naked eye. Once the capillary was in approximately the right position, the light box was closed up and then the fluorescent spot was centered on the pinhole by changing the position of the PMT manually and observing the resultant output signal, which was to be maximized. Sybr Green in water was initially used as a flourophor, but without DNA, the signal intensity was low and subject 
to photobleaching. It was subsequently discovered that moving the capillary so the laser was focused on the polyamide coating produced a strong, consistent fluorescent spot which was easy to align. The capillary could then be repositioned so the capillary window was centered over the laser focus, and experiments could commence.

\subsubsection{Thermal and Visible Light Cameras}

Thermal and visible light images were used to observe the movement of ionic concentration boundaries, bubbles and metal particles in the capillary. Thermal images were obtained with a 10.6 $\mu \mathrm{m}$ Raytheon Control IR2000AS Silicon Bolometer camera. This unit has a fixed focal length and automatic brightness control. An IR transparent AMTIR polymer lens of $38 \mathrm{~mm}$ focal length (Oriel) was placed in front of the camera as a magnifier, giving a field of view of 25 $\mathrm{mm}$. Images were taken every eight seconds, and the Labview data acquisition program was configured to prompt the user to select the region of the IR image containing the capillary at the beginning of the run. Those regions were then cropped out of subsequent images and stored side by side. This image was saved along with separate files containing current and time data, and PMT detector data.

Visible light images were obtained with a Canon Elura DV video camera. For observations of bubble evolution it was aimed through the camera port of a Leica binocular microscope, giving a field of view of about $9 \mathrm{~mm}$. The nickel particles (section 5.4.4) were imaged through the eyepiece of the CE instrument's on-board microscope, giving a field of view of about $3 \mathrm{~mm}$. Data acquisition for the thermal and DV cameras used their NTSC video outputs and a NI-1025 IMAQ card (National Instruments). Two cards were used for simultaneous imaging. The visible light images were cropped and assembled in the same manner as the thermal images. 


\subsubsection{Capillaries, Matrix and Buffer}

Single capillaries extracted from MegaBACE capillary bundles were used so as to closely simulate the MegaBACE itself. Unless otherwise noted, all experiments were conducted with $36 \mathrm{~cm}$ long capillaries, of $75 \mu \mathrm{m}$ diameter, with the detector window $2 \mathrm{~cm}$ from the cathode. While anti-EOF coatings could have been applied to inhouse new capillaries, it was believed the commercial capillaries would be more consistent. The MegaBACE capillary windows were all $5 \pm$ $0.2 \mathrm{~mm}$ long, knowledge of which was useful for alignment purposes. Had these capillaries not been adaptable for our purposes, windows would have had to be burnt in raw capillaries with nichrome wire, and then the surface treatment performed, with the attendant risk of breakage during these steps. Fortunately for our purposes, a large number of MegaBACE capillary bundles were made available when the GSC retired two MegaBACEs so many capillaries were available for cut capillary experiments.

The choice was made to use matrix and buffer from Amersham Biosciences (Part \# US79676), rather than make it from scratch despite uncertainty as to the exact contents. Polymerization of small batches of LPA in-house according to [45] gave highly variable viscosity despite identical starting conditions from batch to batch. The Amersham LPA by contrast was very consistent. The buffer concentration in the matrix was believed to be $50 \mathrm{mmol} / \mathrm{L}$ of each of Tris and TAPS) respectively [60]. What is not known is the type and concentration of neutral denaturant (such as urea) which is normally present in these reagents according to the literature, or what other ions were present in the LPA from the polymerization. This topic is taken up again in C.2. 
Tris and TAPS refer, respectively, to the weak base Tris(hydroxymethyl)aminomethane) and the weak acid [(2-Hydroxy1,1-bis (hydroxymethyl)ethyl)amino]-1-propanesulfonic acid. These buffer components were originally introduced by the Karger lab, who developed LPA for use in sequencing [45]. Tris/TAPS was found to produce better sequence data than the traditional biological buffer TrisBorate, possibly because the former is less likely to cause band broadening by interacting with reagents left over from DNA purification. Tris and TAPS are part of a larger class of "Good's buffers" which exhibit this benign behaviour [52], and which have the further advantage that they are only $50 \%$ dissociated in equilibrium and so have high buffering capacity.

\subsection{Samples and Methods}

\subsubsection{MegaBACE Samples}

Detailed explanations of E. coli based DNA amplification, cell lysis, the cycle sequencing reaction and sample cleanup are given in Appendix A.5. These protocols are used by the GSC preparation and in the Marziali lab. Briefly, a DNA insert from the Mammalian gene collection (MGC-10790) was ligated into a circular M-13 vector (a piece of DNA capable of reproducing inside cells) was grown in $E$. coli. The cells were then lysed and the insert DNA isolated. The cycle sequencing reaction was then performed to produce fluorescently labeled single stranded copies of the original template of monotonically increasing size. The DNA was then purified out and the sample dried down. Before injection on the MegaBACE, samples were resuspended in $20 \mu \mathrm{L}$ of $\mathrm{dH}_{2} \mathrm{O}$, and briefly vortexed and centerfuged.

The same sample preparation protocols were used for bubble propagation experiments conducted in the Marziali lab as well as in the agarose resuspension experiments described in Appendix B.1. In the 
latter experiments, the samples were pooled and realiquoted several times to ensure consistency. Sizing these samples in agarose showed them to consist of 20-1000 bp single stranded sequencing fragments as well as $7.5 \mathrm{~kb} \mathrm{M}-13$ template DNA and some quantity of unseparated long fragments, presumably $E$. coli genomic DNA left over from colony growth. E. coli's genome is $3 \mathrm{Mb}$, so these genomic fragments may be in the kilobase to megabase range.

In the experiments on bubble propagation, the control consisted of MegaBACE 4-Color Sequencing Standard (Amersham Biosciences (US79678), which agarose sizing showed to be free of large fragments. Plates with no DNA, consisting simply of MegaBACE running buffer, were also used as controls.

\subsubsection{MegaBACE Experimental Parameters}

MegaBACE runs were performed in the following manner, except where noted. LPA was injected at 1000psi for 2 minutes with a buffer plate ( $100 \mu \mathrm{L}$ of buffer per well) at the cathode. The LPAcontaining microcentrifuge tubes were then replaced with tubes containing $2 \mathrm{ml}$ of buffer and a 15 minute prerun performed at $9000 \mathrm{~V}$. The buffer plate was then removed, and the cathode array rinsed in $\mathrm{dH}_{2} \mathrm{O}$. Samples resuspended in $20 \mu \mathrm{L} \mathrm{dH}_{2} \mathrm{O}$ were then injected for 10 $\mathrm{s}$ at $3 \mathrm{kV}$ and the cathode array rinsed again. The original buffer plate was restored to the cathode and the electrophoresis ran for 240 minutes at $6 \mathrm{kV}(100 \mathrm{~V} / \mathrm{cm}$ in the $60 \mathrm{~cm}$ capillaries). Typical capillary current at the start of the run was $4 \mu \mathrm{A}$.

\subsubsection{Electropherogram Analysis}

Electropherograms from sequencers such as the MegaBACE are evaluated in terms of read length, which represents the number of 
bases that can be identified with a given level of confidence.

Processing of the four fluorecence data signals is shown in Figure 6.

The basecalling algorithm accepted as standard in most sequencing facilities is called the Phred algorithm $[1,61]$. The algorithm accounts for the differences in mobility shifting of the four colours because of differential drag from the different fluorophors and fitting a Gaussian to each peak based on the expected peak frequency. The "quality" of each base is given by $Q=-10 \log _{10}\left(P_{e}\right)$, where $P_{e}$ is the probability that the base is incorrectly scored. Phred10 bases represent a $90 \%$ confidence that the base has been correctly identified and Phred20, represents a $99 \%$ confidence value. The read length is the number of bases above a desired Phred value. A generally accepted minimum is Phred20, although some assembly algorithms use the lower quality parts of the sequence to aid construction of the contiguous "tiling paths" that make up the genome sequence. 


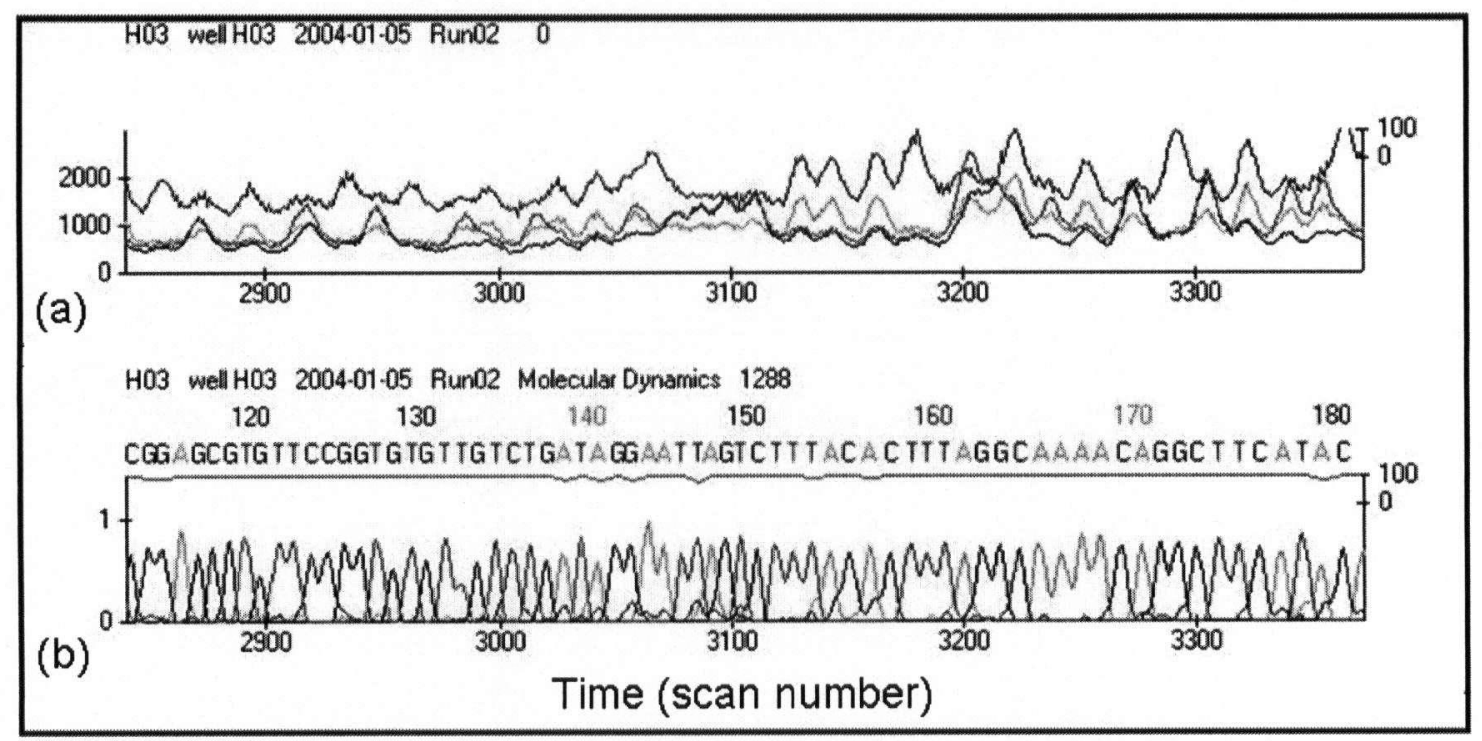

Figure 6 (a) Raw MegaBACE electropherogram fluorescence data, and (b) basecalled with the Phred algorithm. The latter has been normalized, curve-fit and mobility shifted.

A typical example of a successful sequencing run from the MegaBACE is shown in Figure 7. Beyond about $700 \mathrm{bp}$, the peaks become diffusion-broadened enough that base quality drops below the Phred20 cutoff.

The MegaBACE software as shipped did not allow separate files of raw current and PMT signal data to be extracted in order to do detailed analysis. The software's exportable data for basecalling had the initial 20 minutes of pre-DNA-arrival data truncated. Proprietary object files were fortunately obtained from Amersham Biosciences which, in conjunction with a Perl script, allowed extraction of raw current and PMT signal strength data. 


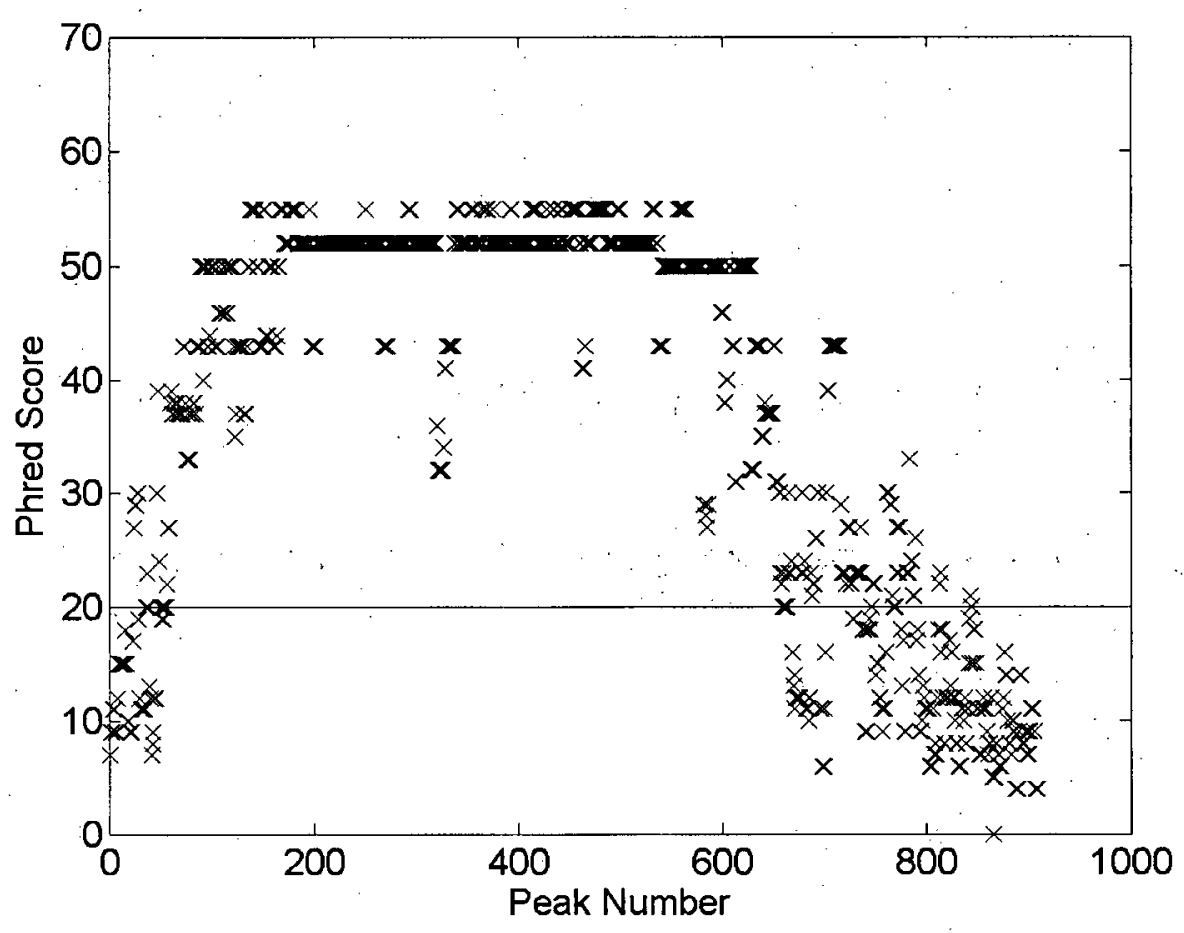

Figure 7 Data from a typical successful electropherogram (Phred20 $=704$ ) showing the tailing off of peak quality as the reads become diffusion limited.

\subsubsection{Single Capillary Instrument Samples}

The challenge with the single capillary instrument was finding a sample capable of producing current decline in a reproducible manner. Cycle sequencing products produced highly variable results. This is true in the MegaBACE as well: Figure 8 , page 44, shows a large range of current decline and Table 3 , page 138, shows a factor of two range in fluorescent signal strength from identically prepared samples. A characteristic of ethanol-precipitated cycle sequence products is relatively low concentrations of DNA with extremely low background electrolyte concentrations. Purification in 96 well plates includes hard- 
to-reproduce steps such as manually tapping the plates against the bench, and variations in time in the introduction of different reagents. As a result, there may be considerable variability in samples across individual plates and from plate to plate [13].

\begin{tabular}{|l|l|l|l|}
\hline Sample & $\begin{array}{l}\text { Conductivity } \\
\mu \mathrm{S} / \mathrm{cm}\end{array}$ & $\begin{array}{l}{[\mathrm{DNA}]} \\
\mu \mathrm{g} / \mathrm{ml}\end{array}$ & $\begin{array}{l}\text { Injection } \\
\text { Coefficient } \\
\mu \mathrm{g} / \mathrm{Q}\end{array}$ \\
\hline $\begin{array}{l}20 \text { to }>50 \mathrm{~kb} \text { Cycle } \\
\text { Sequence Products }\end{array}$ & 25 & 10 & 148 \\
\hline $0.5-50 \mathrm{~kb}$ BAC Fragments & 300 & 130 & 160 \\
\hline $48 \mathrm{~kb} \lambda$ DNA & 700 & 500 & 265 \\
\hline $\begin{array}{l}500 \text { to } 23 \mathrm{kbp} \lambda \text {-Hind III } \\
\text { Ladder }\end{array}$ & 700 & 500 & 265 \\
\hline $500 \mathrm{bp}$ PCR Fragments & 15 & 9 & 250 \\
\hline
\end{tabular}

Table 1 Data for concentration and solution conductivity for different DNA samples. The injection coefficient was calculated from equation (2-10) $\frac{M_{D N A}}{Q_{i n j}}=\frac{\mu_{D N A} C_{D N A} M W_{D N A}}{\sigma_{\text {sample }}}$.

In the search for injection consistency, various other DNA fragments were evaluated. Conductivity and concentration data is shown in Table 1. Two polydisperse samples of high buffer conductivity and DNA concentration were found to cause reproducible current decline. These were 0.5 to $30 \mathrm{~kb}$ bacterial artificial chromosome (BAC) digest fragments from the GSC and $0.5 \mathrm{~kb}$ to $25 \mathrm{~kb} \lambda$ Hind III restriction digest (New England Biolabs). The success of these samples then led to the use of undigested $\lambda$ DNA (New England Biolabs N3011L) for the experiments reported here. At $48 \mathrm{~kb}$, this double stranded, linear phage DNA represents a compromise between $\sim 8 \mathrm{~kb}$ template and 
longer double stranded genomic DNA fragments found in actual sequencing samples and which are believed to be the source of current decline. This commercial product was found to be very consistent and stable, and reproducible results were obtained over a period of three years using samples from three separate lots. In these experiments, $20 \mu \mathrm{L}$ aliquots of $500 \mu \mathrm{g} / \mathrm{mL} \lambda$ DNA with $5 \mu \mathrm{L}$ of $5 x$ Sybr Green intercalating dye were used throughout, although $\lambda$ DNA produced current decline with or without Sybr Green.

One experimental result which lends weight to the use of $\lambda$ DNA as a model fragment was that the time evolution of current decline was found to be similar for all of the fragments tested. In other words, the high concentration, high conductivity $\lambda$ and $\lambda$ Hind III samples exhibited the same behaviour as low concentration, low conductivity cycle sequencing products. This is of significance as it was not initially known whether current decline was an intrinsic property of the enhanced sample stacking associated with the low conductivity of the sequencing samples. The similarity in behaviour of the low and high conductivity samples suggests that the current decline is independent of the degree of sample stacking.

\subsubsection{Single Capillary Instrument Parameters}

As noted, $36 \mathrm{~cm}$ long, $75 \mu \mathrm{m}$ diameter capillaries cut from MegaBACE bundles were used for all experiments. LPA was injected for $60 \mathrm{~s}$ at $500 \mathrm{psi}$. Where noted, a 15 minute prerun was performed with $1 \mathrm{x}$ buffer at the anode rather than LPA. This reduced the starting current from 8-9 $\mu \mathrm{A}$ to $4-5 \mu \mathrm{A}$ at $5000 \mathrm{~V}$. The temperature of the heater plate was $55^{\circ} \mathrm{C}$ and both injection and runs were performed at $5000 \mathrm{~V}$. For runs other than threshold experiments, the injection time was $20 \mathrm{~s}$. The reduced length of the capillary, higher injection voltage, 
heater temperature and longer injection time were all designed to promote current decline or exacerbate any effects such as spontaneous bubble formation which might occur. $5000 \mathrm{~V}$ was also the maximum operating voltage of the machines.

\subsubsection{Cut Capillary and Thermal Image Analysis}

The conductivity distribution in single capillaries was determined by the method of successive cutting and resistance measurement [43] [62]. First, one centimeter of capillary at the anode end was cut off to remove any void caused by matrix shrinkage as the capillary was removed from the heater. The capillary's resistance was then measured by placing it across two buffer baths at $400 \mathrm{~V}$ and measuring the resulting current. Each measurement lasted about three seconds, minimizing changes in the ion distribution profile in the capillary. The measurement was repeated with successive removals of $\Delta x=1 \mathrm{~mm}$ segments from the cathode end of the capillary. Once past the region where the resistance was observed to change rapidly, cut segments were increased to $\Delta x=10 \mathrm{~mm}$. This approach was found to be fast, have good resolution and be much more accurate than pre-cutting the capillary into segments and measuring each individually. The conductivity of the $\mathrm{n}^{\text {th }}$ segment of capillary was found from (3-1) where $A$ is the capillary area $\left(m^{2}\right), V$ and $I_{n}$ are the capillary voltage and current respectively:

$$
\sigma(n)=\frac{\Delta x}{A} \frac{1}{\left(R_{n+1}-R_{n}\right)} \text { where } R_{n}=\frac{V}{I_{n}} .
$$

Capillaries were cut by pushing the edge of a glass slide onto the capillary. (N.B. The slides were prone to shattering; for safety, readily available ceramic cutters should be used in future) Cuts were measured against lines marked on the lab bench, with an estimated error of about $0.25 \mathrm{~mm}$. The length of the capillary was measured 
before and after the series of $1 \mathrm{~mm}$ cuts, and again after the $10 \mathrm{~mm}$ cuts and then the average spacing of each was calculated based on the number of measurements taken. Thus the uncertainties in boundary positions are $\pm 0.5 \mathrm{~mm}$.

As noted, thermal images were assembled by placing cropped images of the capillary side by side to show the temporal evolution of thermal fluctuations. After each run, the average brightness across each individual capillary image was calculated, and the edge(s) of the hot region boundary(ies) were found by comparing the image to a threshold value. A complicating factor was that current decline causes overall cooling, changing the threshold value for the depletion boundary in the image. This was only significant for the longest depleted buffer runs, such as Figure 15(b) but in those cases, the image contrast was adjusted across the time axis so as make the image brightness even. For the visual images, positions of bubbles and nickel particles were determined manually, using a Matlab script. One data point was obtained for each image (every eight seconds) and matrices of these data were saved with the current and run time for each data point. Position and current data were then fitted using Matlab (The Mathworks) and Microsoft Excel, as described further in section 4.4.2. 


\section{Chapter 4 Experiment}

The experiments presented in this chapter constitute a systematic investigation of the mechanism underlying observations of read length degradation with associated current decline. The first goal of this investigation was to establish the relationship between read length and current decline. Having established that current decline directly affects read length, the next objective was to investigate the source of the current decline. The formation of gas bubbles in the capillaries was explored as a possible source and was largely discounted, leaving inhomogeneity in matrix conductivity as the most likely mechanism for current variation. Conductivity profiles of affected capillaries were examined, leading to the discovery of regions of ionic depletion. Further investigation revealed that depletion region formation is linked to propagation velocities of the regions' boundaries, leading to a highly non-linear relationship between quantity of contaminant DNA present and the formation of the depletion region. Experiments were also performed demonstrating that suppression of long contaminant fragments by sample resuspension in agarose improves the success rate of DNA sequencing.

\subsection{Relationship between read failure and capillary current decline.}

In collaboration with the Genome Sciences Center, the correlation between read length and integrated current throughout the run (total loaded charge) was measured for DNA sequencing samples prepared according to section 3.3.1. The samples were resuspended in $\mathrm{dH}_{2} \mathrm{O}$, injected for 10 seconds at $3 \mathrm{kV}$, and run for 240 minutes at 6 $\mathrm{kV}$. Figure 8(a) shows that there are many shortened reads in this sample set, and that read length increases with increasing total loaded 
charge (TLC) until 700-800bp at which point read length is diffusion limited [63].
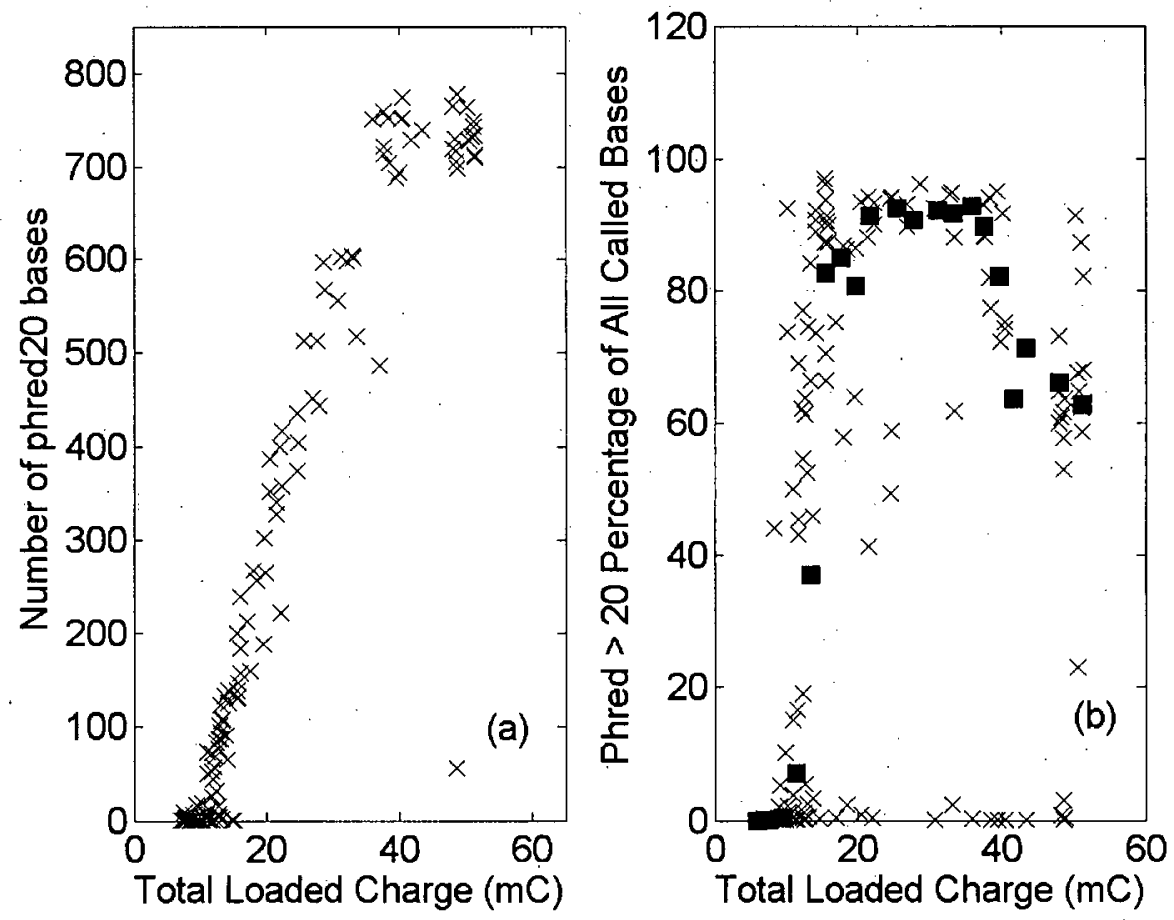

Figure 8 (a) Read length vs total loaded charge for samples resuspended in $\mathrm{dH}_{2} \mathrm{O}$. Each " $\mathrm{x}$ " is one capillary - approximately 200 capillaries are represented. (b) The same data shown as percentage of all called bases with Phred quality values $\geq 20$ vs. total loaded charge (TLC) after a $4 \mathrm{~h}$ run. Each (x) represents one capillary and $(\boldsymbol{})$ are mean values for TLC binned into $2 \mathrm{mC}$ increments.

Figure 8 (b) shows the same data as Figure $8(a)$ but is presented as read quality vs. total loaded charge. Read quality is expressed as the number of bases called with Phred (confidence) values $>20$ as a percentage of all called bases. Runs with TLC $<15 \mathrm{mC}$, show either poor basecalling of very broad, late-arriving peaks, or spurious basecalls where no real peaks are present. Between 15 and $40 \mathrm{mC}$, the number of Phred $>20$ bases is directly proportional, and nearly 
equal, to the number of bases passing the detector. In this range, reduced read lengths resulting from low electrophoresis current are not associated with poor base quality, but simply fewer fragment bands arriving at the detector. Runs with TLC $>40 \mathrm{mC}$ are resolution limited above 700 Phred $>20$ bases. These runs, like the run shown in Figure 7 , have a tail of lower quality bases beyond 700bp which is why the percentage of good reads declines despite the actual Phred20 read length being very good.

We conclude that decreasing current during electrophoresis is directly responsible for poor read lengths by decreasing the number of DNA fragment bands arriving at the detector, but that detrimental effects of current decline do not include direct degradation of the analyte band quality. Virtually all called bases in runs with TLC between 15 and $40 \mathrm{mC}$ have scores of Phred20 or higher, indicating that lower current reduces the number of fragment bands passing the detector, but does not significantly decrease the quality of those bands. This is consistent with the source of current decline being upstream of, i.e. nearer the cathode than, the sequencing fragments.

\subsection{Bubbles}

\subsubsection{The Contribution of Bubbles to Current Decline}

As discussed in section 2.5, spontaneous bubble nucleation seems to have been mitigated by sequencing through use of non cross-linked matrices and lower fields [43] [10]. Anecdotal evidence for bubbles in commercial capillary array machines has been reported more recently however. In particular, the MegaBACE showed current variability on the timescale of seconds (see Figure 46 (b)), consistent with bubble behaviour observed in our single capillary instrument and reported by Swerdlow [42]. We, therefore, undertook to find out if bubbles were to be found in MegaBACE capillaries, whether they 
were, in fact, spontaneously nucleating, how they might otherwise appear, and how they might propagate.

In order to observe bubble effects in the MegaBACE, four plates of samples made according to Section 3.3.1, were injected at $3000 \mathrm{~V}$ for $10 \mathrm{~s}$ and run at $6000 \mathrm{~V}$ for 240 minutes in the MegaBACE in the Marziali Lab. Control plates of Amersham DNA size standard and DNA-free running buffer were also injected and run in the same manner. It should be emphasized that the sequencing samples contain sequencing fragments and long DNA fragments, whereas the size standard contains sequencing fragments but no long contaminant DNA. After each run, the air box door was opened, and with both ends of the capillary still submerged in buffer, the capillaries were cooled from $40^{\circ} \mathrm{C}$ to room temperature in less than a minute. Each sixteencapillary bundle was then removed for inspection. Bubble lengths were estimated by visual comparison to a ruler under a binocular microscope. A variety of bubbles were observed, including unbroken bubbles of 5 to $70 \mathrm{~mm}$ length, "bubble trains" of one or two capillary diameter bubbles spaced a few diameters apart over up to $100 \mathrm{~mm}$, and single scattered bubbles at various points in the capillary. For the purposes of this analysis, however, only the presence or absence of bubbles was noted. 


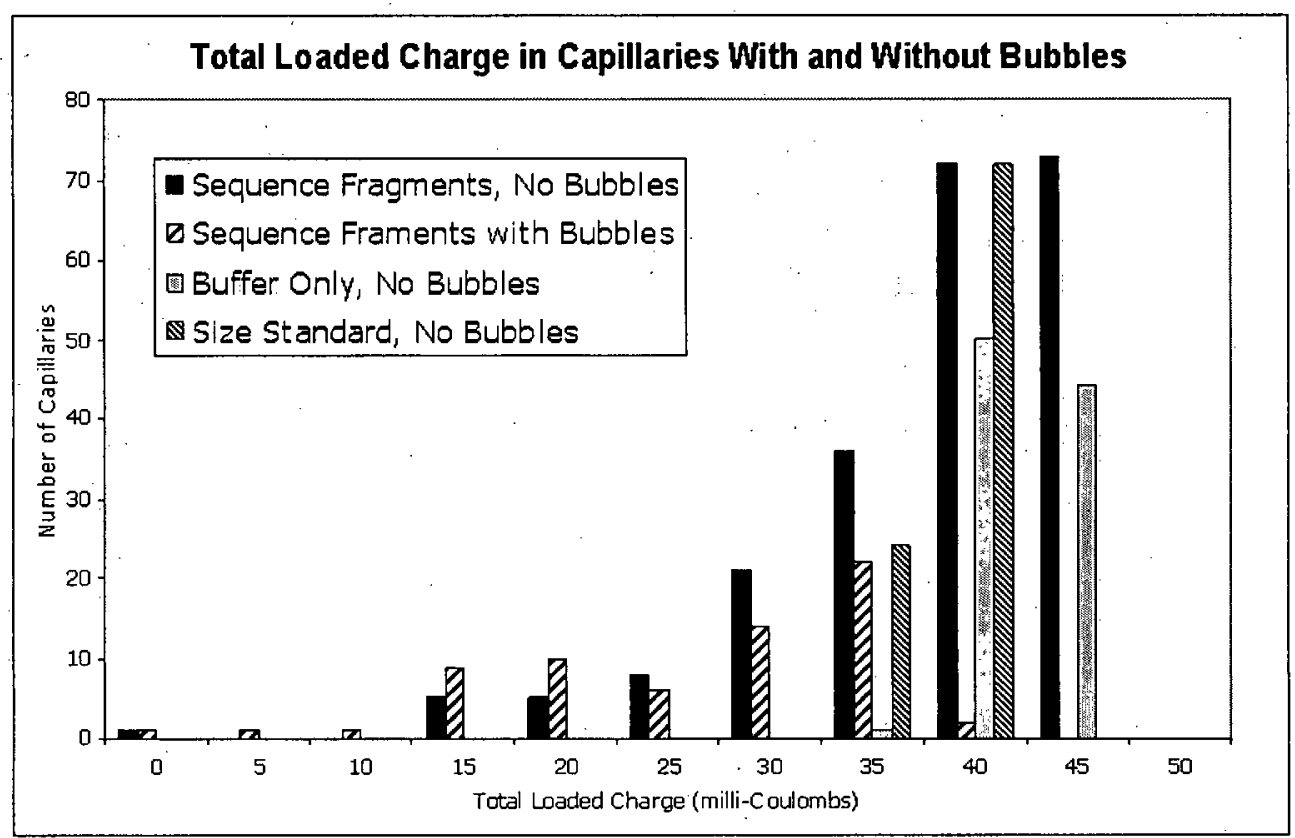

Figure 9: Total loaded charge of capillaries with and without bubbles. See text below for details

Figure 9 shows a histogram of the capillaries binned by total loaded charge. The mean TLC for the size standard control was $42 \mathrm{mC}$ and $43 \mathrm{mC}$ for the buffer-only control. None of the capillaries in either of the two control plates showed any post-run bubbles. Capillaries with sequencing fragments (380 working capillaries total) were divided into those which contained bubbles and those that did not. As $97 \%$ of the buffer control samples showed a TLC of $40 \mathrm{mC}$ and above we chose this as a figure of "normal" TLC and values below that represent "reduced" TLC

For plates containing sequencing samples - the black and striped bars in Figure $9-51 \%$ of the runs showed normal loaded charge, while $22 \%$ showed reduced TLC and bubble formation, and $26 \%$ showed reduced TLC without bubble formation. Clearly, there is a process unrelated to bubble formation that is also capable of 
reducing the total loaded charge. This process may furthermore be the dominant source of current decline even for the capillaries containing bubbles. Experiments with artificially introduced bubbles indicate that the bubbles alone are probably not sufficient to produce the observed current decline. This topic is taken up again in Appendix $C$.

\subsubsection{Bubble Formation and Growth.}

Though it was expected that heat dissipation and thermal gradients in the capillaries might allow bubbles to grow or migrate, it remained unclear whether these bubbles formed spontaneously, or were externally introduced. This question was addressed with the single capillary instrument. With the capillary positioned so the cathode end could be observed through the flat wall of a $100 \mu \mathrm{L}$ square well cut from a 384 well polystyrene culture plate, the state of the capillary entrance could be observed before, during and after sample injection. $\lambda$ DNA samples, as per section 3.3.4, were injected and run for periods from 5 to 120 minutes, and the capillary was afterward removed for inspection under the microscope. In thirty-nine consecutive runs, if no bubbles were observed immediately before injection of long DNA fragments, no bubbles were observed after injection or at the end of the runs.

In the absence of spontaneous bubble formation, bubbles must form while the capillary entrance is exposed to air, and this was indeed found to occur. Bubbles could be reliably introduced by allowing the capillary to cool with the cathode exposed. The matrix would shrink, forming a meniscus at the cathode, and a bubble would then form when the cathode was returned to buffer. Critically, such a thermal fluctuation with exposed capillaries was found to occur in the MegaBACE during normal cathode plate exchanges. Thermocouple measurements at the MegaBACE's cathode plate holder revealed that 
through the matrix injection and pre-run stage, with all machine doors closed, the temperature in the cathode plate holder rose to $24^{\circ} \mathrm{C}$ as a result of heat transfer from the air box immediately above the cathode plate which circulates air around the capillaries at $40^{\circ} \mathrm{C}$. Upon lowering the cathode plate and opening the drawer to exchange the buffer and sample plates, the air around the exposed capillary array dropped back to room temperature within ten seconds. $22 \mathrm{~mm}$ of each capillary is exposed to this temperature change. Based on a measured coefficient of thermal expansion for the matrix (see Appendix D.2), it was calculated that the matrix would shrink by at least $40 \mu \mathrm{m}$, enough to form a bubble half a capillary diameter in size. Bubbles of such a size were observed to be capable of expansion during sample injection in the single capillary instrument.

It is clear from the these results that local cooling is the most likely mechanism by which bubbles form in sequencers, and could therefore be mitigated through improved temperature control. As current decline is shown to occur in the absence of bubbles, we conclude that a second effect, unrelated to the presence of bubbles, is the principal cause of current decline. Further analysis of bubble behaviour, particularly the relationship of bubbles to ionic depletion regions and the effect of bubbles on current is presented in Appendix C.

\subsection{Ion Distribution during Current Decline}

\subsubsection{Formation of a Depletion Region}

In the absence of bubbles, current decline was reliably produced through injection of sufficient quantities of $\lambda$ DNA. Figure 10 shows cumulative resistance and local conductivity profiles obtained by 
the capillary cutting method introduced in section 3.3.6, for four representative capillaries: one control with no DNA injected and three with $\lambda$ DNA run for the times indicated. Approximately $25 \mathrm{ng}$ of $\lambda$ DNA was injected in each case. The conductivity profiles indicate that the presence of the $\lambda$ DNA caused the formation of an expanding region of ionic depletion which locally decreased the conductivity of the capillary. This local decrease in conductivity is an excellent candidate cause of the current decrease observed during runs.

A striking feature of these plots is the abruptness of the conductivity boundaries, and the low conductivity of the depletion region. For clarity, we refer to the part of the capillary between the cathode (injection end) and the depletion region as the "cathode region", and the remainder of the capillary between the depletion region and the anode as the "anode region". Capillaries cut after varying run times showed the leading and trailing edge of the depletion region (anode and cathode boundary respectively) moving into the capillary with the leading edge moving faster, causing the depletion region to expand. Though the width of the depletion region increased with time during electrophoresis, there was no observed correlation between the length of the run and the actual conductivity value in each region. In other words, variations in conductivity in the background or depletion regions appeared to arise from properties of individual capillaries and did not evolve in time. Only the relative sizes of the different regions changed. 

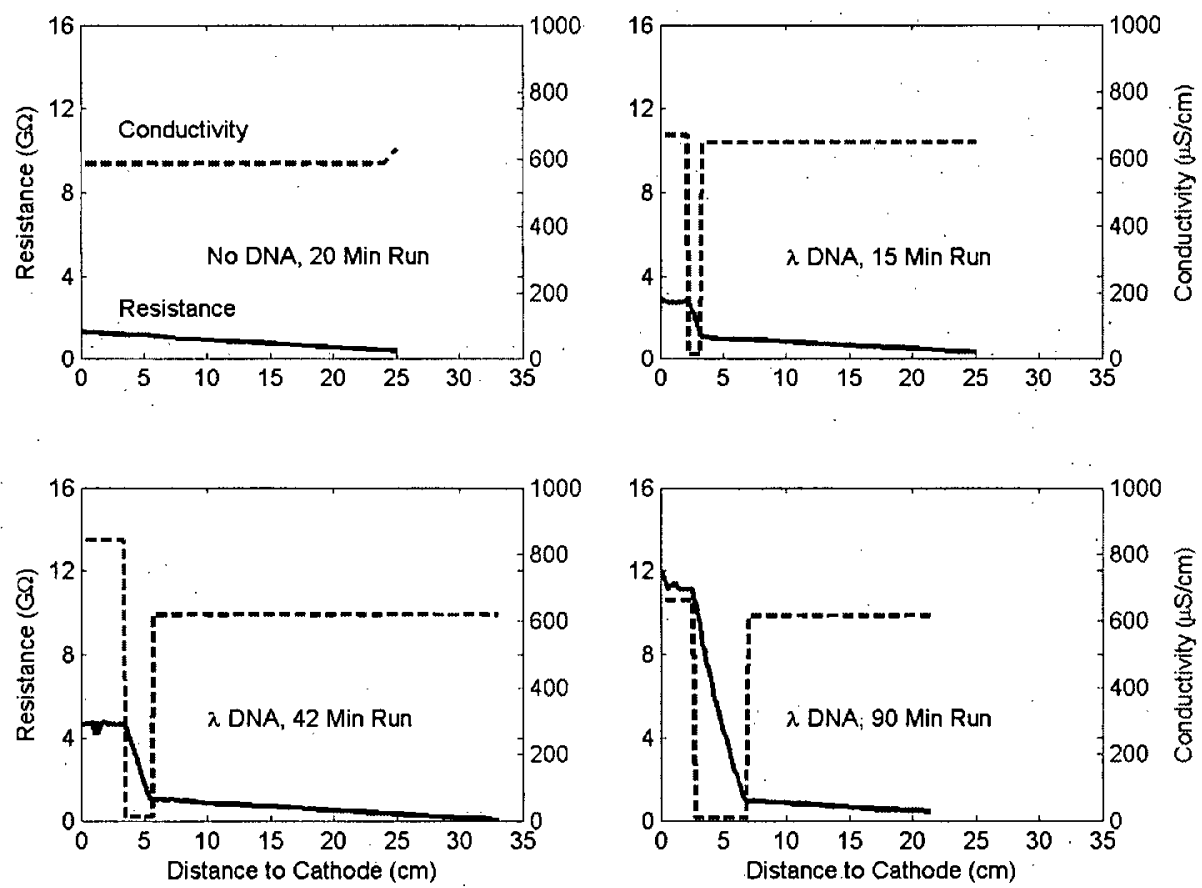

Figure 10 Local conductivity (dashed line) and capillary resistance (solid line) profiles for four cut capillaries. The capillary resistance is the electrical resistance of the remaining capillary, and therefore must decrease as the capillary is cut from the cathode end, and should go to zero at $36 \mathrm{~cm}$. Conductivity is found from making a least-squares fit to each straight segment of the resistance and using that slope as $\Delta R / \Delta x$ in equation 3. $25 \mathrm{ng}$ of $\lambda$ DNA was injected and run for the times indicated.

The resolution of the capillary cutting method was $1 \mathrm{~mm}$ and transitions at each boundary of every run were abrupt enough that the change in slope between two straight regions could be localized to one data point. This indicates that the boundaries are self sharpening: the boundary. propagation mechanism works against diffusion. A Gaussian peak widens according to [64] 


$$
F W H M^{2}=16 \ln (2) D t
$$

Given a diffusion constant of $D \sim 5 \times 10^{-10} \mathrm{~m}^{2} \mathrm{~s}^{-1}$ for the background electrolyte ions, an infinitely sharp transition would be expected to spread to $\sim 3 \mathrm{~mm}$ wide by 30 minutes and $\sim 4.5 \mathrm{~mm}$ after an hour. Instead, sharp boundaries of less than $1 \mathrm{~mm}$ in width were found for runs up to 320 minutes.

There are of course differences between measuring capillary resistance while the capillary is running at high voltage and measuring resistance using a lower voltage while cutting it up. In the former case the capillary is warmer from both internal joule heating and the instrument heater, so the capillary resistance is lower. As a test of the validity of the measured conductivity values, the final experimentally measured resistance could be reconstructed by integrating the measured conductivity over the length of the capillary using the conductivity values found by capillary cutting:

$$
R_{\text {final }}=\frac{1}{T_{c} A}\left[\int_{0}^{c b} \frac{d x}{\sigma_{\text {cathode }}}+\int_{c b}^{a b} \frac{d x}{\sigma_{\text {depletion }}}+\int_{a b}^{L} \frac{d x}{\sigma_{\text {anode }}}\right]
$$

where $c b$ is the cathode boundary of the depletion region and $a b$ is the anode boundary. $A$ is the capillary cross-sectional area and $T_{c}$ is a correction factor for temperature-induced conductivity change. The current was experimentally found to rise $30 \%$ as the heater raised the central $25 \mathrm{~cm}$ of capillary from $20^{\circ} \mathrm{C}$ to $55^{\circ} \mathrm{C}$ so the value of $T_{c}$ was set at 1.3. Figure 11 shows the expected final currents from (4-2) (immediately before removal of the capillary), compared to an experimentally observed temporal current profile which is typical of these experiments. The calculated and measured values were indeed found to be in close agreement for runs from five to 320 minutes in length. Likewise the initial current of each run could be correctly inferred by extrapolating the anode-side conductivity back to the full 
length of the capillary (not shown). Clearly, in these capillaries the formation of the depletion region is the source of the current decline.

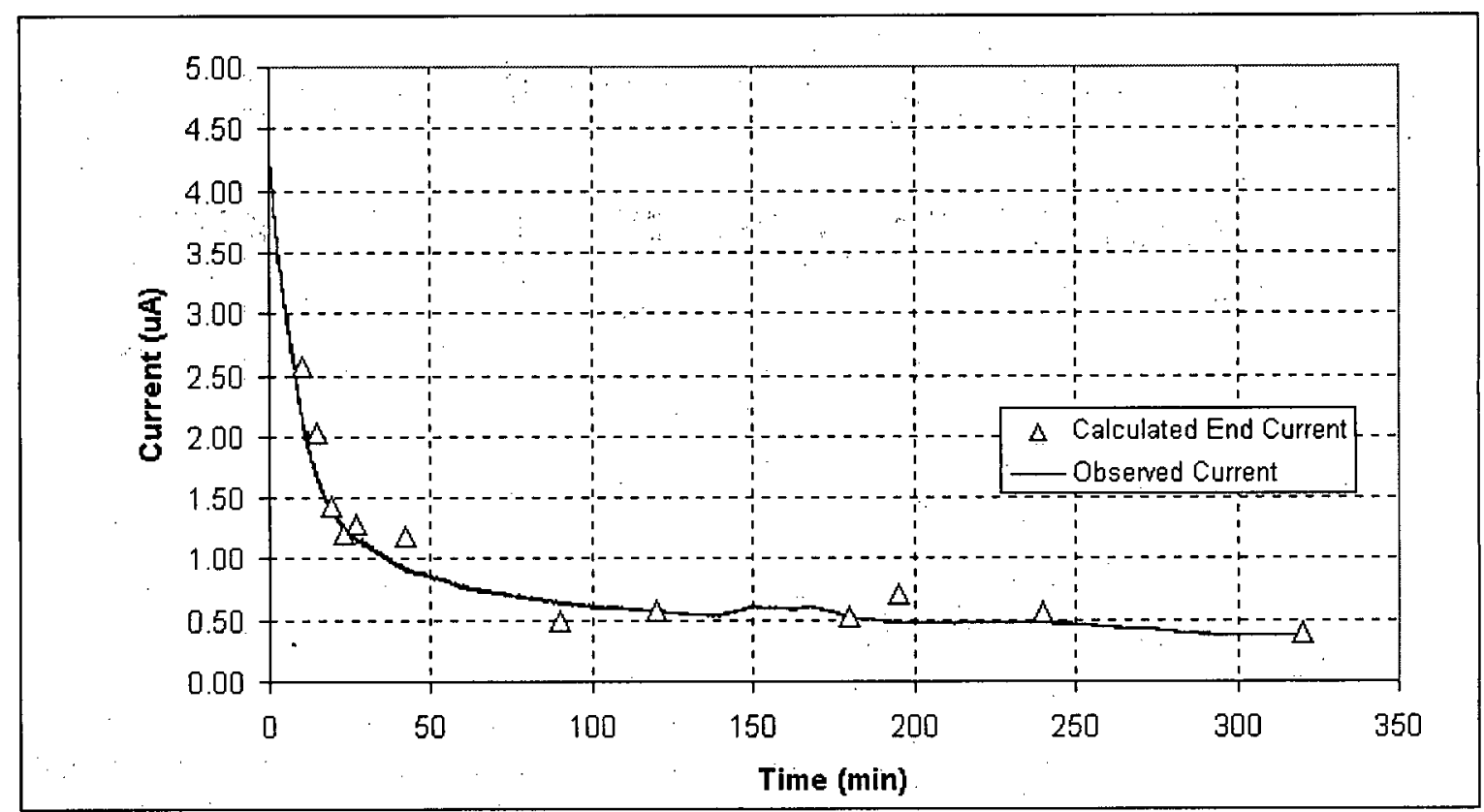

Figure 11 Current at the end of runs of varying duration $(\Delta)$ inferred from ionic concentration profiles for 12 capillaries. The current plot is a continuous 320 -minute run on a single capillary.

\subsubsection{A Threshold for Current Decline}

To establish the relationship between quantity of injected DNA and depletion region formation, $\lambda$ DNA and 500 bp control samples generated by PCR (see section 3.3.4) were injected for periods of five to twenty seconds at $5000 \mathrm{~V}$, and run for five minutes at $5000 \mathrm{~V}$. The results, shown in Figure 12 display a striking threshold in the onset of current decline with the injection of more than approximately $10 \mathrm{ng}$ of $\lambda$ DNA. Below this value, current decline was not observed, and no quantity of 500 bp control DNA caused current decline. Current decline was also not observed when no DNA was injected, save 
specific circumstances such as replacing LPA with buffer at the anode, as described in Appendix C.2

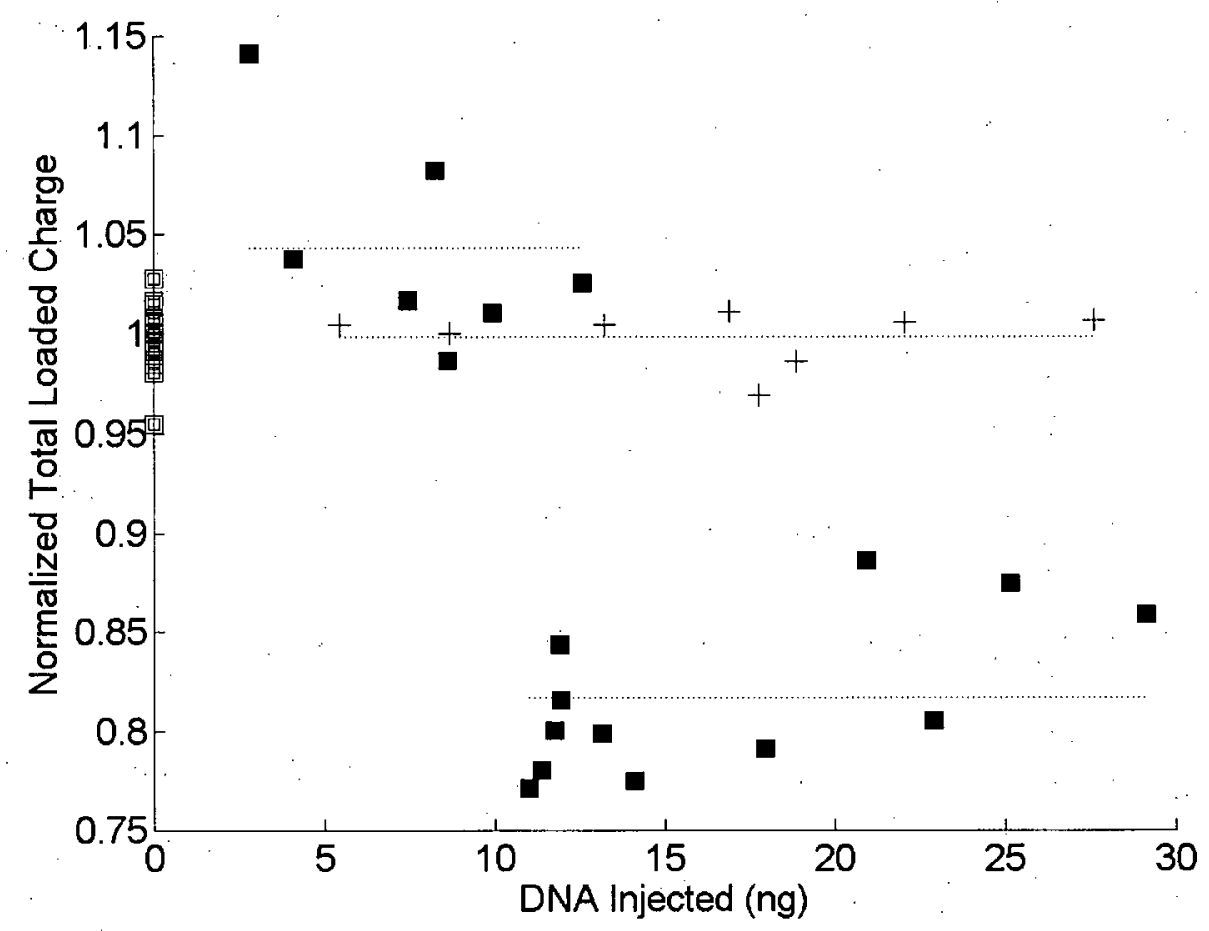

Figure 12 Total loaded charge vs. quantity of injected DNA over a five minute run. The charge in each run was normalized to its initial current value to correct for variability in capillary resistance. For $\lambda$ DNA (a) the current remains stable up to $10 \mathrm{ng}$ injected DNA, but above this threshold the current begins to fall. 500 bp PCR fragments (+) do not cause current decline at any quantity tested. ( $\square-$ on the y-axis) 18 runs where no DNA was injected show no current decline. Dashed lines represent mean values for the $\lambda$ DNA above and below the threshold and for the 500 bp control fragments.

\subsection{Temporal Evolution of Ion Depletion Boundaries.}

While cutting the capillaries provided excellent spatial data on the depletion region, thermal imaging made it possible to observe the 
depletion region evolving in time. As a nondestructive technique, it also allowed repetitive observations with the same capillary, where cutting required a new capillary for each observation. It was initially hypothesized that the temperature change itself might cause boundary propagation. It is shown in section 5.4 that this is unlikely to be the case. Here we discuss only the observed movement of the region of elevated temperature. A discussion of temperature measurement techniques is found in Appendix E, and Figure 49 shows an individual image of the capillary with a region of elevated temperature. To produce Figure 13 below, multiple images of the capillary collected at eight second intervals were cropped, aligned and joined, as explained in section 3.2.3, forming a composite image of the temporal evolution of the thermal profile. Concurrently recording the fluorescent signal allowed determination of the location of the DNA with respect to the elevated temperature region. 

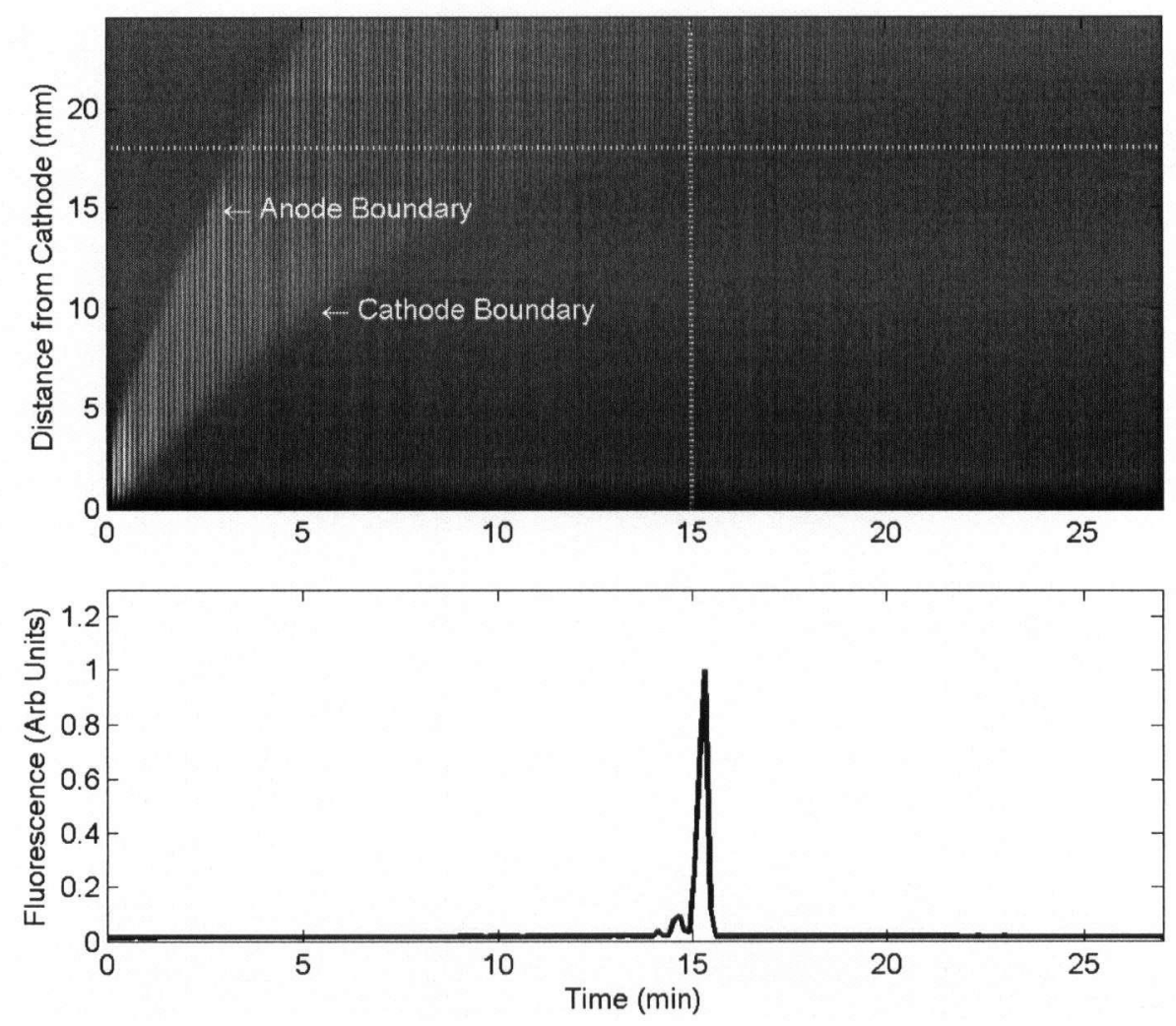

Figure 13 (Top) A series of infrared images of the capillary taken eight seconds apart and displayed side by side. The warmer (brighter) region (initially elevated by about $7^{\circ} \mathrm{C}$ ) corresponding to the depletion region can clearly be seen. The horizontal dotted line corresponds to the position of the fluorescence detector along the capillary. The vertical dotted line represents the point in time the fluorescence peak appeared on the detector. (Bottom) The fluorescence signal acquired simultaneously with the infrared images. The DNA peak appears as the cathode boundary crosses the fluorescent detector's position in the thermal image.

\subsubsection{Cathode Boundary and DNA Movement}

Figure 13 shows a series of thermal images of the capillary over time, displayed side by side. The region of elevated temperature in the 
capillary associated with increased Joule heating in the ionic depletion region is clearly visible. In nineteen runs, (four with DNA, and the rest with depleted cathode buffer as in section 4.4.2) the capillary was cut up after being imaged with the thermal camera. In all of these cases, the position of the ionic depletion region found by capillary cutting was the same as seen in the last thermal images, given allowances for thermal shrinkage as the capillary was removed for cutting.

The fluorescence detector was also used with thermal imaging to determine the location of the $\lambda$ DNA within the conductivity profile. The $\lambda$ DNA was consistently found to be at the cathode-side depletion region boundary, also shown in Figure 13. In some cases a small fluorescence peak was observed at the anode-side depletion region boundary, but no signal was observed in the depletion region. We conclude therefore that the cathode boundary propagates with the DNA.

\subsubsection{Boundary velocity and its relation to depletion region depth.}

As a first step in understanding the temporal evolution of the depletion region, we investigated the role that the depletion depth plays in this region's motion. In particular, we investigated the relationship between boundary velocity and depletion region depth. Since boundary velocity evolves with time, for the purposes of this comparison, boundary motion is characterized by the dependence of the velocity on the capillary current. In the simplest model, we assume the boundary propagation rate is expected to be linear with current and assign it a mobility $\mu$. It should be noted that this, for now, is simply a useful characterization of the motion and is not intended to describe an 
underlying mechanism. For a capillary with a local conductivity $\sigma$, the boundary velocity is

$$
\frac{d x}{d t}=\frac{\mu I(t)}{\sigma A}
$$

This can be integrated to

$$
x(t)=\frac{\mu}{\sigma A} \int_{0}^{t} I(\tau) d \tau x(t)=\frac{\mu}{\sigma A} Q(t)+x_{o}
$$

The boundary propagation is then characterized by the coefficient $\mathrm{C}_{1}$ which can be found by a least squares fit to the data

$$
x(t)=C_{1} Q(t)+C_{2}
$$

where $C_{1}=\frac{\mu}{\sigma A}, C_{2}=x_{o}$ is an offset related to the camera position, and $Q(t)$ is the total loaded charge. $C_{1}$ is expressed in $\mathrm{m} / \mathrm{C}$.

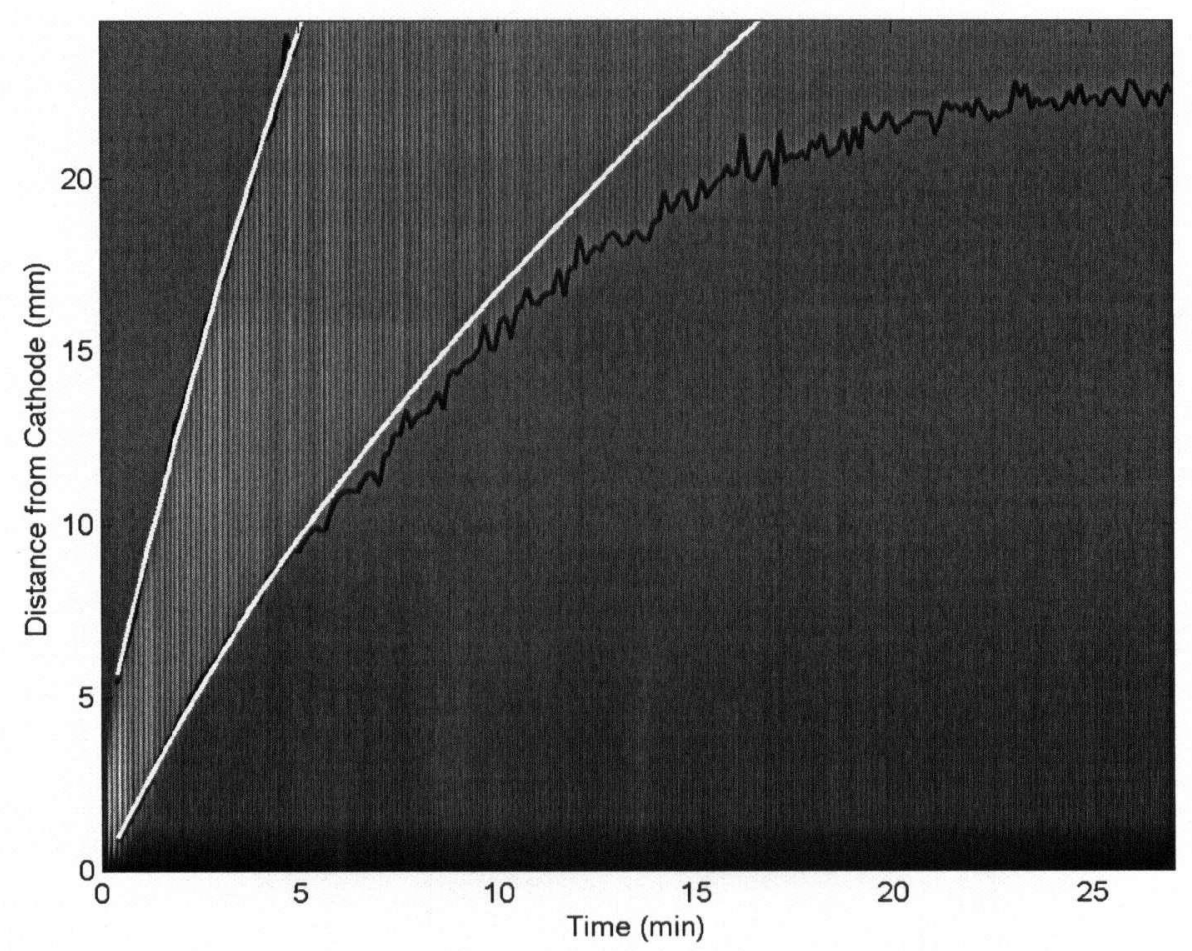

Figure 14 Fits (in white) to the first five minutes of anode and cathode boundary data (in black) using $x(t)=C_{1} Q(t)+C_{2}$. The divergence 
of the cathode boundary from linearity is taken up in detail in section

\section{3.}

- This model is a good fit to the anode boundary, but a relatively poor fit to the cathode boundary. Figure 14 shows equation (4-5) fitted to the first five minutes of anode and cathode boundary data. The distance traveled by the cathode boundary is clearly not linear with total loaded charge; its mobility must have some field dependence. The latter is taken up in greater detail in section 5.3. In contrast, the anode boundary propagation distance is close to linear with charge. Eleven fits to $\lambda$ DNA-induced anode boundaries gave $x(t) \propto Q(t)^{0.97 \pm .15}$.

Propagation of the DNA-induced anode-side boundaries varied from 5.4 to $11 \mathrm{~m} / \mathrm{C}$ for normal matrix, and 10 to $21 \mathrm{~m} / \mathrm{C}$ when the matrix was depleted by running the anode in buffer (see section 3.3.5). The variation was greatest from capillary to capillary, with individual capillaries exhibiting relatively constant propagation rates over multiple runs.

Cut capillary measurements showed that injection of $\lambda$ DNA produced very low depletion region conductivities, on the order of $2-5 \%$ of the initial conductivity. In order to understand the relationship between depletion region depth and boundary propagation rate, different degrees of depletion were needed. This was achieved by running the capillary with normal LPA in the capillary and anode, but diluted cathode buffers and no DNA. With buffers diluted by at least $50 \mathrm{x}$, a moving boundary was visible with the infrared camera. Data was obtained for $1 / 50 x$ and $1 / 100 x$ Tris/TAPS buffer, and $\mathrm{dH}_{2} 0$. These capillaries were also cut up to find the actual conductivity profile. Representative examples are shown in Figure 15. 
Like the DNA induced anode boundaries, propagation rates are linear with charge. Thirteen such runs with run times of 5 to 25 minutes gave a mean exponent of $x(t) \propto Q(t)^{0.99 \pm .3}$.
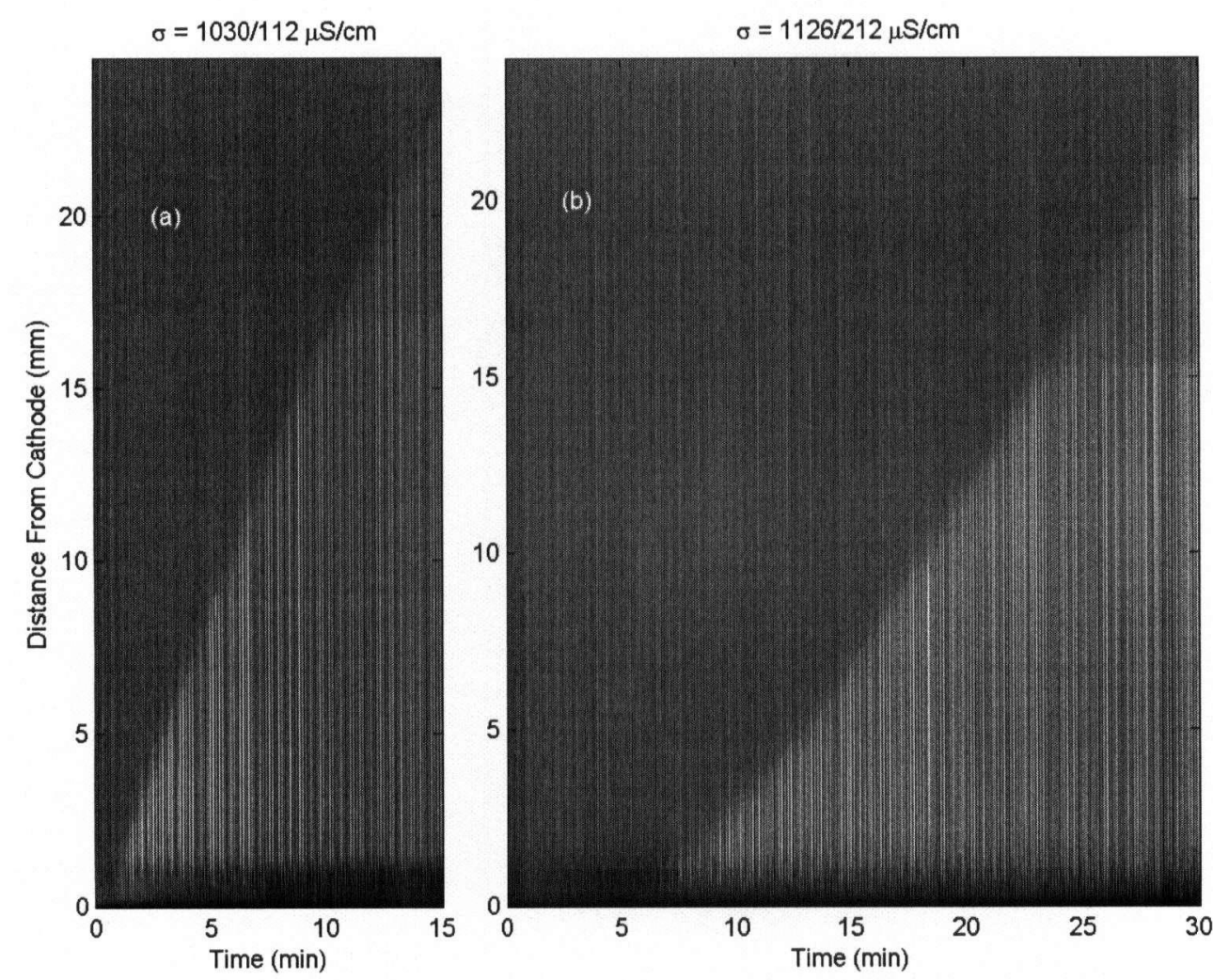

Figure 15 Two examples of ionic depletion boundaries propagating from a low conductivity cathode with no DNA present. (a) The cathode well has $\mathrm{dH}_{2} \mathrm{O}$ and the boundary propagates at $3.5 \mathrm{~m} / \mathrm{C}$ and (b) the well has $0.02 \times$ Tris/TAPS buffer and the boundary propagates at $1.8 \mathrm{~m} / \mathrm{C}$.

Figure 16 shows the relationship between the anode boundary propagation rate and depth of various depletion regions. The propagation rate of $\lambda$ DNA in the absence of current decline is also shown. The only anode-side boundaries which move faster than the $\lambda$ 
DNA are at the front of deep depletion regions, beyond anything achievable by running depleted buffers at the cathode.

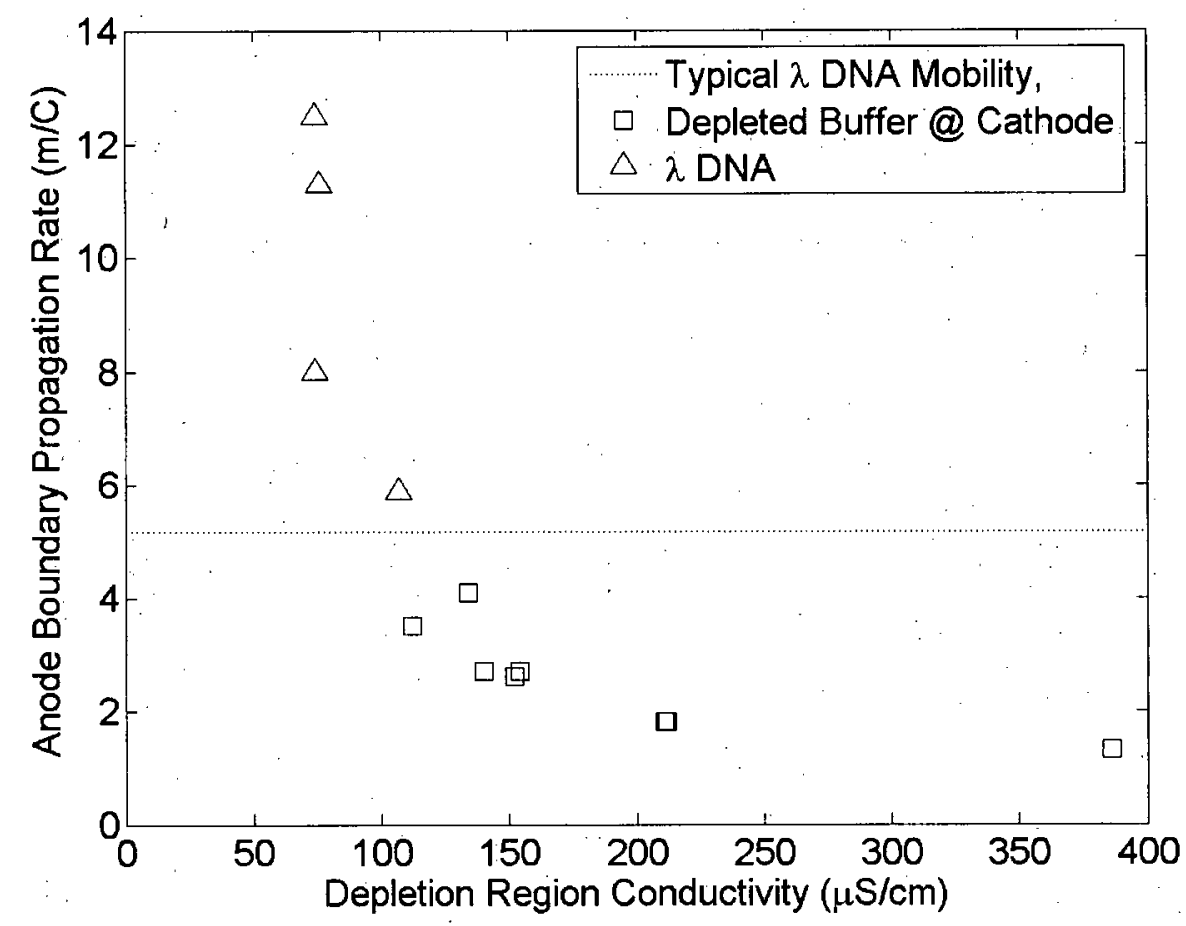

Figure 16 Measured propagation rates for boundaries of different depletion depths. The background conductivity in each case is $1100 \mu \mathrm{S} / \mathrm{cm}$.

As an extension of the discovery of a threshold for DNA injection to cause current decline, thermal imaging was used to investigate the relationship between boundary propagation and the amount of DNA injected. Eighteen $\lambda$ DNA aliquots were injected for varying times using the same capillary, and the rate of anode boundary propagation recorded. The results are shown in Figure 17. As in Figure 12 , the threshold for the onset of depletion is between 10 and $20 \mathrm{ng}$ of DNA, but once established, the anode boundary propagation rate is constant. 


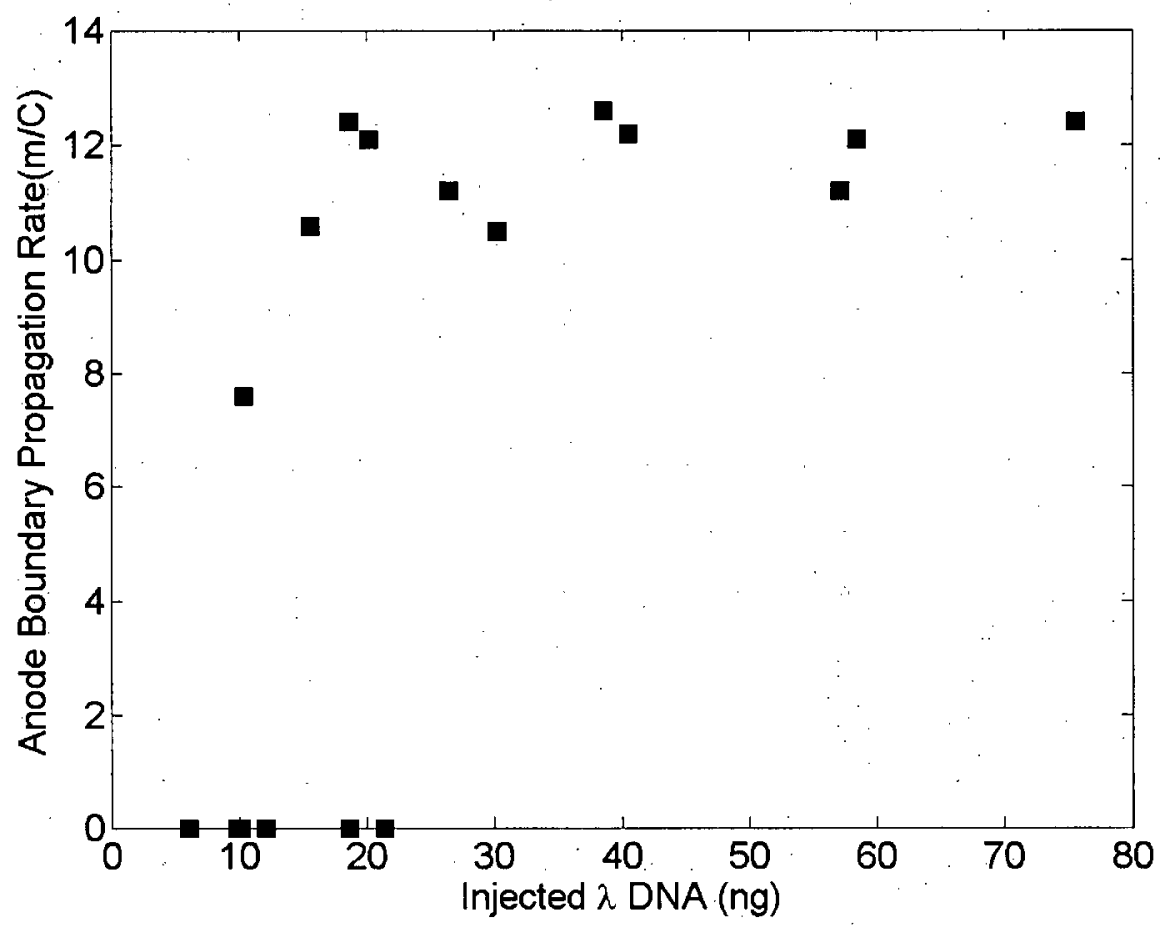

Figure 17 Rate of anode-side boundary propagation vs. quantity of injected DNA. Beyond the threshold for depletion region formation, the anode-side boundary moves at a constant rate. All runs were approximately five minutes.

\subsection{A Mechanism for Current Decline.}

Figure 16 suggests a mechanism for current decline in the presence of large fragments. Even if any quantity of DNA may be capable of locally depleting the background carriers, if the depletion is not deep enough, the DNA moves faster than any induced anode-side boundary so the depletion region cannot form. The expanding depletion region can only form if there is sufficient depletion of the background electrolyte so that the anode-side boundary takes off and runs away from the DNA. Figure 17 suggests that the sufficiently fast anode boundary propagation may only occur when depletion region 
reaches some limit of "total" depletion. That is why adding more $\lambda$ DNA does not cause the anode boundary to propagate faster.

A notable property of this mechanism is that the long DNA fragments do not prevent the movement of small ions by mechanically plugging the capillary, nor by virtue of being a principal current carrier with very low mobility. Nor is the DNA observed to precipitate in the capillary [13]. Rather, the long fragments propagate with their usual mobility, but current decline occurs because of the runaway anodeside boundary, the propagation of which is independent of, though driven by, the long DNA fragments. 


\section{Chapter 5 Analysis of Boundary Propagation}

We have shown in the previous chapter that current decline is caused by the DNA forming a depletion region which, having reached sufficient depth, is able to expand because the anode-side boundary starts to propagate faster than the DNA and cathode-side boundary. We have also demonstrated a relationship between the depth of the depletion region and the rate of anode boundary propagation in the absence of DNA. It remains then to be understood why the depletion region forms and why the anode and cathode side boundaries move as they do.

In this chapter, analytic arguments are used to show how slow moving ions can form a depletion region downstream. A numerical model is introduced by which the quantity of DNA required to completely deplete the background electrolyte is calculated. The movement of the boundaries is analyzed, starting with the cathode boundary. Comparison of the anode and cathode boundaries allows us to discard variable electroosmotic flow as a mechanism for the cathode boundary non-linearity. Fits to the cathode-side boundary movement shows the DNA to transition between two modes of migration as the field declines.

Analysis of the anode boundary propagation mechanism is less conclusive as the experimental evidence is not definitive. The theory of moving boundaries for different electrolyte types is therefore introduced to provide a framework for possible mechanisms. A. numerical model is introduced to test various possibilities. It is shown that the like-ion conductivity gradient mechanism which has been cited as the likely source of these slow moving, low conductivity boundaries 
$[43,62,65]$ is not in fact likely to be at work, and it is concluded that the most likely mechanism requires some combination of fixed charge in the matrix and/or a rise in $\mathrm{pH}$ in the depletion region so $\mathrm{OH}^{-}$ions become principle carriers. Experimental evidence for $\mathrm{pH}$ changes, via changes observed in the electrophoretic behavior of nickel particles, is presented in section 5.4.4.

\subsection{Formation of the Depletion Region}

The common approach to analytical models of capillary zone electrophoresis is to assume that the conductivity of background electrolyte is $\sim 100$ times higher than the conductivity of macromolecules being analyzed [66]. This permits the assumption that the electric field in equation (5-1) is constant, and thus linearized, the governing equations become tractable. If the electrophoresis scheme does not involve a high degree of sample concentration, such an assumption is quite reasonable, but this is not true of the present case. If, for example, the $20 \mathrm{~s}$ wide peak in Figure 13 contains $\sim 10 \mathrm{ng}$ of DNA, the concentration is $\sim 70 \mathrm{mmol} / \mathrm{L}$, comparable to the concentration of the electrolyte, and enough to significantly perturb the conductivity. It is still instructive, however, to examine the perturbation of a small quantity of slow-moving charged species on the background carriers.

Consider again the 1-D Smoluchowski equation to describe the time evolution of ion concentrations in the presence of diffusion and electric drift:

$$
\frac{\partial C_{i}(x, t)}{\partial t}=D_{i} \frac{\partial^{2} C_{i}(x, t)}{\partial x^{2}}+\frac{\partial}{\partial x}\left(\mu_{i} E(x, t) C_{i}(x, t)\right)
$$

where $C_{i}, D_{i}$ and $\mu_{i}(C)$ are respectively the concentration, diffusion coefficient and electrophoretic mobility of the $\mathrm{i}^{\text {th }}$ charged species. In 
the present case, the buffer ions are all univalent, so the charge $z$ is not included.

$$
\begin{gathered}
\sigma(x, t)=\sum_{i} F\left|\mu_{i}\right| C_{i} \\
J(t)=V\left[\int_{b}^{t} \frac{d x}{\sigma(x, t)}\right]^{-1} \\
E(x, t)=\frac{J(t)}{\sigma(x, t)} .
\end{gathered}
$$

The local field $E(x, t)$ is found by first calculating the local conductivity, $\sigma(x, t)$, where $F$ is the Faraday constant and the summation is over all ion species, and then the current density $\mathrm{J}(\mathrm{t})$ from (5-3). For ionic polymers such as DNA, $C_{d n a}$ refers to the concentration of charges, not individual particles, and thus includes the multiplicity of charges per molecule. The fact that these are long molecules is reflected in the low mobility but not in the concentration. The matrix buffer is $50 \mathrm{mmol} / \mathrm{L}$ Tris-TAPS, initially at $\mathrm{pH}=8.3$, (see section 3.2.4, page 33 for more detail). They dissociate to give $25 \mathrm{mmol} / \mathrm{L}$ of TAPS ${ }^{-}$and Tris $\mathrm{H}^{+}$, so the contribution of $\mathrm{H}^{+}$and $\mathrm{OH}^{-}$ $\left(5 \times 10^{-6} \mathrm{mmol} / \mathrm{L}\right.$ and $2 \times 10^{-3} \mathrm{mmol} / \mathrm{L}$ respectively at $\left.\mathrm{pH} 8.3\right)$ can be ignored and we model a two-ion system plus DNA. Charge conservation dictates $C_{+}=C_{-}+C_{d n a}$.

For further simplification, we consider the system in the frame of reference of the DNA, so that $C_{d n a}(x)$ is not time dependent. In reality, the mobility of the large DNA is of order $1 / 10$ that of the carrier ions so we can assume the DNA is fixed. Let us now consider the quantity of interest - the concentration of mobile ions $C=C_{+}+C_{+}$. From charge conservation,

$$
\begin{gathered}
C(x, t)=2 C_{-}(x, t)+C_{d n a}(x) \\
\frac{\partial C(x, t)}{\partial t}=2 \frac{\partial C_{-}(x, t)}{\partial t} .
\end{gathered}
$$


Re-writing (5-1) for all mobile ions, taking advantage of (5-5) and assuming the quantity of injected DNA is small, and that

$$
\mu=\mu=-\mu_{+}
$$

we can write

$$
\frac{\partial C_{-}}{\partial t}=D \frac{\partial^{2} C_{-}}{\partial x^{2}}+\frac{D}{2} \frac{\partial^{2} C_{d n a}}{\partial x^{2}}+\frac{1}{2} \frac{\partial}{\partial x}\left(\mu E C_{d n a}\right) .
$$

For initial conditions of a uniform anion concentration, and ignoring diffusion for now, the drift term of (5-6) will initially tend to increase $C$. on the cathode side of the DNA peak (where $d C_{d n a} / d x>0$ ) and decrease $C$. on the anode side of the peak. Note also that a larger carrier ion mobility will exacerbate this effect. Assuming that $C_{d n a}$ and the resulting perturbation to $C_{\text {. (defined as }} \delta C_{\text {-) }}$ ) is also small we can follow a perturbation analysis similar to that used in the study of membranes with fixed charges [66]:

$$
C_{-}(x, t)=C_{-}^{0}+\delta C_{-}(x, t)
$$

and

$$
\frac{\partial \delta C_{-}}{\partial t}=D \frac{\partial^{2} \delta C_{-}}{\partial x^{2}}+\frac{D}{2} \frac{\partial^{2} C_{d n a}}{\partial x^{2}}+\frac{J}{2 F} \frac{\partial}{\partial x}\left(\frac{C_{d n a}}{2 C_{-}^{0}}\right) .
$$

At steady state, using boundary conditions at the ends of the capillary, and assuming that $C_{d n a}(x)$ is nonzero over a finite length $\mathrm{x}_{1}<\mathrm{x}<\mathrm{x}_{2}$ and

$$
\delta C_{-}(0)=\delta C_{-}(L)=0,
$$

where $L$ is the length of the capillary. This yields:

$$
\delta C_{-}(x)=-\frac{J}{4 F C_{-}^{0} D}\left(1-\frac{x}{L}\right) \int_{0}^{L} C_{d n a}(\tau) d \tau .
$$

for all $x>x_{2}$ downstream of the $C_{d n a}$ peak. Although (5-10) is an approximation for small perturbations only, it sheds light on what drives the initial formation of the depletion region, and, as is shown below, on the threshold behaviour of current decline as a function of increasing injected DNA. In qualitative agreement with observations and 
numerical results shown below, (5-10) predicts increasing ionic depletion downstream of the low mobility DNA, with the amount of depletion proportional to the total number of low mobility fragments. As we shall see in section 5.2, numerical solutions to (5-6) indicate that the depletion $\delta C_{\text {., }}$ once it becomes a significant fraction of $\mathrm{C}_{.}^{\circ}$, grows rapidly in magnitude with increasing amounts of injected DNA as a result of the growing electric field in the depleted region. Therefore, formation of a deep depletion region tends to occur rapidly once a critical quantity of low mobility fragments is reached. Estimates of this critical number using (5-10) where we set $\delta C_{-} \sim C^{\circ}$ and given typical run parameters: $\mathrm{C} .=25 \mathrm{mmol} / \mathrm{L}, \mathrm{D}=5 \times 10^{-10} \mathrm{~m}^{2} / \mathrm{s}, \mathrm{J}=1000 \mathrm{~A} / \mathrm{m}^{2}$ gives $\mathrm{C}_{\mathrm{DNA}} \sim 10^{12}$ nucleotides, or $\sim 1 \mathrm{ng}$ of large (i.e. slow moving) DNA fragments. Beyond this critical number, increasing DNA injection will rapidly deepen the depletion region to the point where, as demonstrated in Figure 12, the depletion region boundary mobility exceeds the fragment mobility and the depletion region expands. Experimental results from Figure 12 indicate that this threshold occurs at $\sim 10 \mathrm{ng}$ of injected DNA.

\subsection{Numerical Modeling of lonic Depletion}

We turn now to numerical modeling of the CE system. Complete models of CE are difficult to produce as the system's behaviour is dependent on properties which vary widely with chemical components. Most schemes are also subject to numerical diffusion, which can result from errors in transport due to the finite size of the discretization and can dominate over Brownian diffusion. The difficulty in removing numerical diffusion without making models somewhat opaque has been mentioned as a limitation [67]. Models are likely to be most useful where, such as in the present case, they are used to better understand a limited set of observed phenomena, or predict to 
first order the behaviour of well-understood analytes in well-buffered systems [68]. CE has been modeled with both continuum and molecular dynamics equations [69]. An attempt was made here to remove numerical diffusion by calculating electric fields based on charge imbalances. This worked until proper constants were applied at which point the model became totally unstable. Such an approach is discussed in [70] where it is pointed out that the discretization step size must be on the order of the Debye length which is impractically small for real simulations. The following simulation uses a first order finite difference approximation (FDA) to the continuum Smoluchowski equation.

The purpose of the present model is to show the effect of DNA on background ions and to test hypotheses related to moving boundaries. This of course means that the background electrolyte concentration and resulting electric fields are not constant. It also means that acid base equilibrium must be maintained, which represents the majority of computation time in this simulation. Several simplifying assumptions can be made however. Electroosmotic flow is neglected as it has been shown experimentally to first order to be unaffected by evolution of the depletion region as discussed in 5.4.4.

Diffusion shall also be neglected, for two reasons. It is on the order of 100 times slower than the drift terms in the transport equations [71], and in this model, Brownian diffusion is actually overwhelmed by numerical diffusion. Fortunately, the experimental results we seek to explain here, such as in Figure 10, show that the boundaries of interest are self-sharpening, so in these cases, the system will act to negate the effect of numerical diffusion. In the absence of electroosmotic flow or any other hydrostatic effects, the flow profile is taken to be flat across the capillary so the discretization can be one dimensional. 
The behaviour of ions under changes in concentration, temperature and $\mathrm{pH}$ is complex and varies from species to species. Of greatest concern are relationship of mobility to temperature and concentration: $\mu(T)$ and $\mu(C)$, and $p H(T)$, the variation of $\mathrm{pH}$ with temperature. In a localized region of the capillary, $T \propto 1 / \sigma \propto 1 / \mathrm{C}$ so $\mu(T)$ and $\mu(C)$ are coupled (see Appendix E), which may be of significance in the case, addressed in section 5.4.2, of motion of boundaries between identical solutions of different concentrations. In the simulations presented in section 5.4.2, ionic mobilities are made functions of concentration.

In the case that changes in $\mathrm{pH}$ mediate boundary movement, discussed in section 5.4.3, $p H(T)$ could become significant. In fact, $d p H / d T$ for $50 \mathrm{mmol} / \mathrm{L}$ Tris-TAPS was experimentally found to be $-0.02 /{ }^{\circ} \mathrm{C}$. Given that the largest temperature increase measured in the depletion region was about $7^{\circ} \mathrm{C}$, this represents a fall of $0.14 \mathrm{pH}$ units which is not significant compared to rise in $\mathrm{pH}$ of 2-3 units believed to be occurring in the depletion region. For this reason the temperature dependence of the $\mathrm{pH}$ is not included in the simulation. 

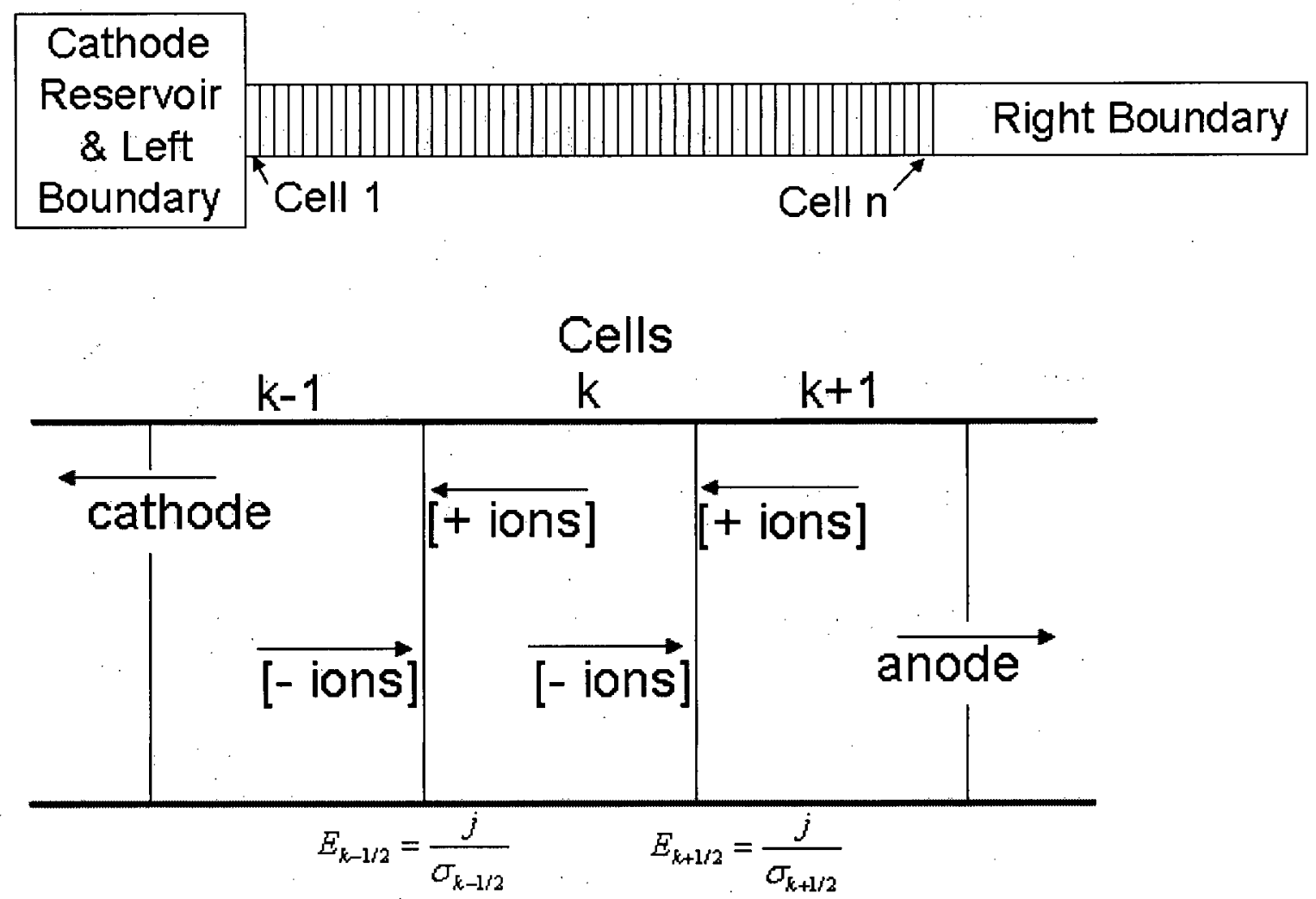

Figure 18 1-D discretization of the capillary showing ion flows in the upwind scheme.

Figure 18 shows how the capillary is discretized in such a way as to preserve the charge neutrality required by equation (5-1). When voltage is applied, the positive ions move to the left and the negative ions move to the right. The charge flux per unit time of a given ion is defined as

$$
\Theta_{i}=\mu_{i} C_{i} E A
$$

where $A$ is the area of the capillary. Charge neutrality is explicitly preserved by defining the electric field at the cell boundaries $E_{k \pm 1 / 2}$ such that the net charge from the movement of ions into and out of 
each cell is zero. Equating the negative and positive species flowing into and out of cell $k$ gives

$$
\sum_{i-} \mu_{i-} C_{i-}^{k-1} E_{k-1 / 2}-\sum_{i-} \mu_{i-} C_{i-}^{k} E_{k+1 / 2}=\sum_{i+} \mu_{i+} C_{i+}^{k+1} E_{k+1 / 2}-\sum_{i+} \mu_{i+} C_{i+}^{k} E_{k-1 / 2}
$$

where each sum is over the $i$ species of each charge. Combining like fields and expressing in terms of the conductivity gives

$$
\left[\sum_{i-} \mu_{i-} C_{i-}^{k-1}+\sum_{i+} \mu_{i+} C_{i+}^{k}\right] \frac{j}{\sigma_{k-1 / 2}}=\left[\sum_{i+} \mu_{i+} C_{i+}^{k+1}+\sum_{i-} \mu_{i-} C_{i-}^{k}\right] \frac{j}{\sigma_{k+1 / 2}} \text {. }
$$

Given a constant current $j$ at each time step, this expression holds if

$$
\begin{aligned}
& \sigma_{k-1 / 2}=F\left[\sum_{i-} \mu_{i-} C_{i-}^{k-1}+\sum_{i+} \mu_{i+} C_{i+}^{k}\right] \\
& \sigma_{k+1 / 2}=F\left[\sum_{i+} \mu_{i+} C_{i+}^{k+1}+\sum_{i-} \mu_{i-} C_{i-}^{k}\right] .
\end{aligned}
$$

The left side of equation (5-1) is written in discrete form as

$$
\frac{C_{k}^{\text {new }}-C_{k}^{\text {old }}}{\Delta t}=\nabla(\mu E C)
$$

Here we define the field gradient for positive ions as

$$
\frac{\Delta E}{\Delta x}=\frac{E_{k+1 / 2}-E_{k-1 / 2}}{\Delta x}
$$

and the concentration gradient for positive ions as

$$
\frac{\Delta C}{\Delta x}=\frac{C_{k+1}-C_{k}}{\Delta x} \text {. }
$$

Applying (5-16) and (5-17) to (5-15)and simplifying gives:

$$
\frac{C_{k}^{\text {new }}-C_{k}^{\text {old }}}{\Delta t}=\frac{\mu_{k+1} E_{k+1 / 2} C_{k+1}-\mu_{k} E_{k-1 / 2} C_{k}}{\Delta x},
$$

For positive species and for negative species: 


$$
\frac{C_{k}^{\text {new }}-C_{k}^{\text {old }}}{\Delta t}=\frac{\mu_{k+1} E_{k-1 / 2} C_{k-1}-\mu_{k} E_{k+1 / 2} C_{k}}{\Delta x} .
$$

Equations (5-18)and (5-19) preserve charge neutrality throughout.

Chemical equilibrium is often ignored in CE simulations, because the assumption $\mathrm{C}_{0} \gg \mathrm{C}_{\text {analyte }}$ implies that the $\mathrm{pH}$ is constant and under normal conditions, $\mathrm{H}^{+}$and $\mathrm{OH}^{-}$ions can be ignored. In the present case however, the contribution of these species must be taken into account as buffer capacity may be exceeded and water ions potentially become significant current carriers. As discussed in section 3.2.4, the buffer solution consists of a mixture of Tris, a weak monovalent base, and TAPS, a weak monovalent acid. The coupled equations of chemical equlibrium can then be written as

$$
\begin{gathered}
k_{\text {Tris }} H^{+} \\
k_{\text {TAPS }}=\frac{[\text { Tris }+x]\left[H^{+}+x+y+z\right]}{\left[\text { Tris } H^{+}-x\right]} \\
k_{w}=\left[H^{+}+x+y+z\right]\left[O H^{-}+y\right]\left[H^{+}+x+y+z\right] \\
{[\text { TAPS }-y]}
\end{gathered}
$$

Here $\mathrm{K}_{\mathrm{TrisH}^{+}}$is the conjugate acid dissociation constant for Tris, $\mathrm{k}_{\text {TAPS }}$ is the acid dissociation constant for TAPS and $x, y$ and $z$ are the contributions to the $\mathrm{H}+$ concentration arising from the dissociation of Tris $\mathrm{H}^{+}$, TAPS and $\mathrm{H}_{2} \mathrm{O}$ respectively. The initial conditions are applied as concentrations of neutral Tris, TAPS and $\mathrm{H}^{+}$and $\mathrm{OH}^{-}$at $10^{-7} \mathrm{M}$, This system of non-linear equations is solved for $x, y$ and $z$ using Newton's method at each iteration, and the concentrations of the species are updated with the new values of $x, y$ and $z$.

The requirement to simulate acid-base equilibrium makes the model both more and less complicated. It can be assumed that because $\mathrm{H}_{2} \mathrm{O}$ is present at $55 \mathrm{M}$, it is in infinite supply everywhere. The 
mobility of water ions is also not well defined and while $\mathrm{H}+$ and $\mathrm{OH}^{-}$ mobilities are nominally given in Table 2 , ion hopping makes those values difficult to define precisely [72]. Because the supply of water is infinite however, the concentrations of the water ions can be calculated by the chemical equilibrium equations. This is convenient as the mobility of those ions is so high as to make numerical stability difficult to achieve without impractically small time steps. In fact, the same results were obtained when the water ions were moved with finite difference equations, as when chemical equilibrium calculations were used to determine their concentrations. The chemical equilibrium calculation appears to correct for any instability introduced by the finite difference scheme for the water ions. This approach might break down if all the buffer ions were truly depleted, but in fact even when the capacity of the buffer to maintain a stable $\mathrm{pH}$ is exceeded, the conductivity is still dominated by the movement of one or other of the main ions and the distribution of neutral species.

The capillary was divided into $n=256$ cells, of $0.1 \mathrm{~mm}$ each, using the natural $1-D$ discretization following from the radial symmetry of the system in the absence of bulk flow such as EOF. The time step was then chosen as $10 \mathrm{~ms}$ so the maximum movement of the $\mathrm{TrisH}^{+}$ ions is less than $0.07 \mathrm{~mm}$ per time step. This was found to produce stable simulations at any degree of ionic depletion. The cathode reservoir then naturally represents the left boundary, the contents and volume of which can be either held constant or altered as needed. The anode side boundary is held at the initial conditions as it is assumed that conditions there remain fixed throughout the simulation.

The algorithm was implemented in both Matlab and Fortran77 with data presentation performed in Matlab. The algorithm was as follows

1. Initialize concentration matrix with undissociated buffer 
2. Set initial DNA conditions, balancing DNA charge with added TrisH $\mathrm{H}^{+}$.

3. Calculate ionic equilibrium in each cell

4. Calculate total capillary resistance and current

5. Calculate conductivity, electric field and molecule velocity.

6. Apply upwind and downwind FDA equations to positive and negative species

7. Calculate chemical equilibrium in each cell.

Repeat steps 4-7 as needed.

All simulations were run with a $36 \mathrm{~cm}$ capillary of $75 \mu \mathrm{m}$

diameter, at $5000 \mathrm{~V}$. Ion properties are listed in Table 2:

\begin{tabular}{|l|l|l|}
\hline Species & $\begin{array}{l}\text { Electrophoretic } \\
\text { Mobility }\end{array}$ & Equlibrium \\
\hline Tris $^{+}$ & $30.1 \times 10^{-9} \mathrm{~m}^{2} \mathrm{Ns}[73]$ & $\mathrm{pKb}=8.1[74]$ \\
\hline TAPS $^{-}$ & $25 \times 10^{-9} \mathrm{~m}^{2} \mathrm{Ns}\left[{ }^{\star}\right]$ & $\mathrm{pK}=8.4$ (Sigma Aldrich) \\
\hline $\mathrm{H}^{+}$ & $363 \times 10^{-9} \mathrm{~m}^{2} \mathrm{Ns}[74]$ & $\mathrm{pKw}=14$ \\
\hline $\mathrm{OH}^{-}$ & $205 \times 10^{-9} \mathrm{~m}^{2} \mathrm{Ns}[74]$ & pKw $=14$ \\
\hline $\begin{array}{l}\text { DNA (free } \\
\text { solution) }\end{array}$ & $37 \times 10^{-9} \mathrm{~m}^{2} \mathrm{Ns}[27]$ & $\begin{array}{l}\text { N.B: This is for any } \\
\text { length DNA }\end{array}$ \\
\hline
\end{tabular}

Table 2 Physical data for the numerical model. Measurement of TAPS mobility is show in D.1

Two versions of the model were developed, one with all the carrier ions and neutral species [TrisH ${ }^{+}$, TAPS $^{-}, \mathrm{H}^{+}, \mathrm{OH}^{-}$, Tris $^{\circ}$, TAPS $^{\circ}$ ] and $\mathrm{DNA}^{-}$in which acid-base equilibrium is maintained, and another with two or three ions (i.e., one ion of each charge or two ions of like charge and a counterion) behaving as fully dissociated strong electrolytes. The ions' mobilities and concentrations were then chosen to resemble TrisH $^{+}$, TAPS ${ }^{-}$and/or faster and slower ions as needed. The latter, non-buffered model, which runs much faster due to the 
absence of the chemical equilibrium calculation, serves as an approximation for when the electrolyte is not depleted, the $\mathrm{pH}$ is near neutral and the water ions do not play a major role.
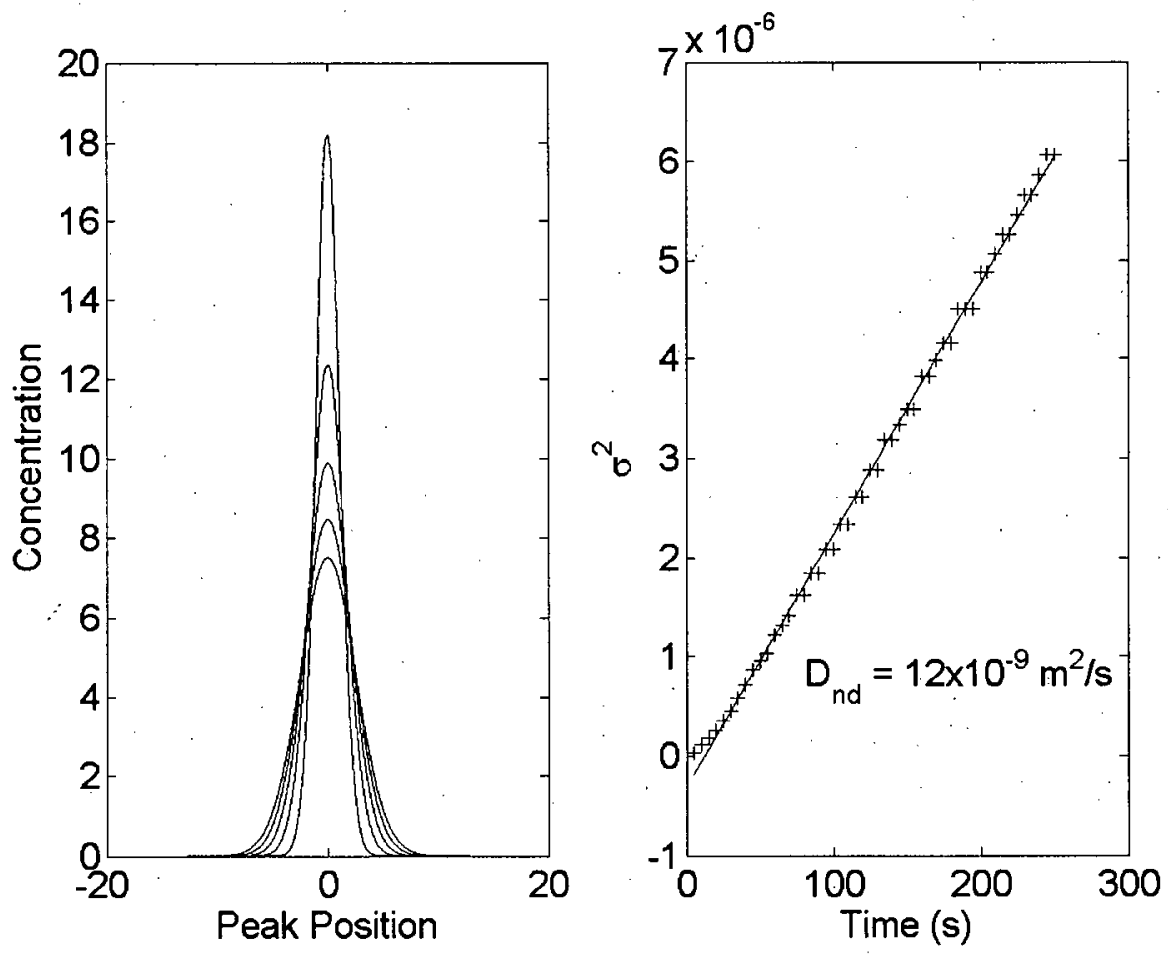

Figure 19 Non-buffered simulation of the decay of a peak in ion concentration over 250 seconds due to numerical diffusion.

The first order finite difference approximation used in this model exhibits numerical diffusion. The effective diffusion constant can be found by initially setting the concentration of the ions so that there is a gaussian peak. As the simulation proceeds, the center remains fixed but the peak shrinks and spreads out maintaining a constant area. A diffusion constant [22] can be found through the evolution of the peak width over time:

$$
\sigma_{\text {peak }}^{2}=\frac{F W H M^{2}}{8 \log (2)}=2 D\left(t+t_{o}\right)
$$


Figure 19 shows a plot of $\sigma^{2}$ vs. time from which the numerical diffusion constant is found to be $D_{n d}=12 \times 10^{-9} \mathrm{~m}^{2} / \mathrm{s}$. The diffusion constant of ions can be found from the Einstein diffusion relation:

$$
D=\frac{\mu k T}{e}
$$

where $e$ is the elementary electronic charge. At room temperature, we find $D \sim 0.025 \mu$ which gives values of $D \sim 5 \times 10^{-10} \mathrm{~m}^{2} / \mathrm{s}$ for the main carrier ions and $5 \times 10^{-11} \mathrm{~m}^{2} / \mathrm{s}$ for $\lambda$ DNA. While this dominance of numerical diffusion does not negate the usefulness of the model for qualitative experiments, care must be taken to ensure the numerical diffusion does not produce significant errors. If, for example, a DNA peak is introduced as in Figure 20, but given a non-zero mobility, numerically diffused DNA will "leak" into the downstream depletion region and cause the anode side boundary to propagate away in accordance with equation (5-41), giving an incorrect result. The solution in this case is to run the simulation in the frame of reference of the DNA peak, as was done in the analytic case. In this manner the. DNA position will not be recalculated using the FDA, so it does not have a chance to diffuse. 

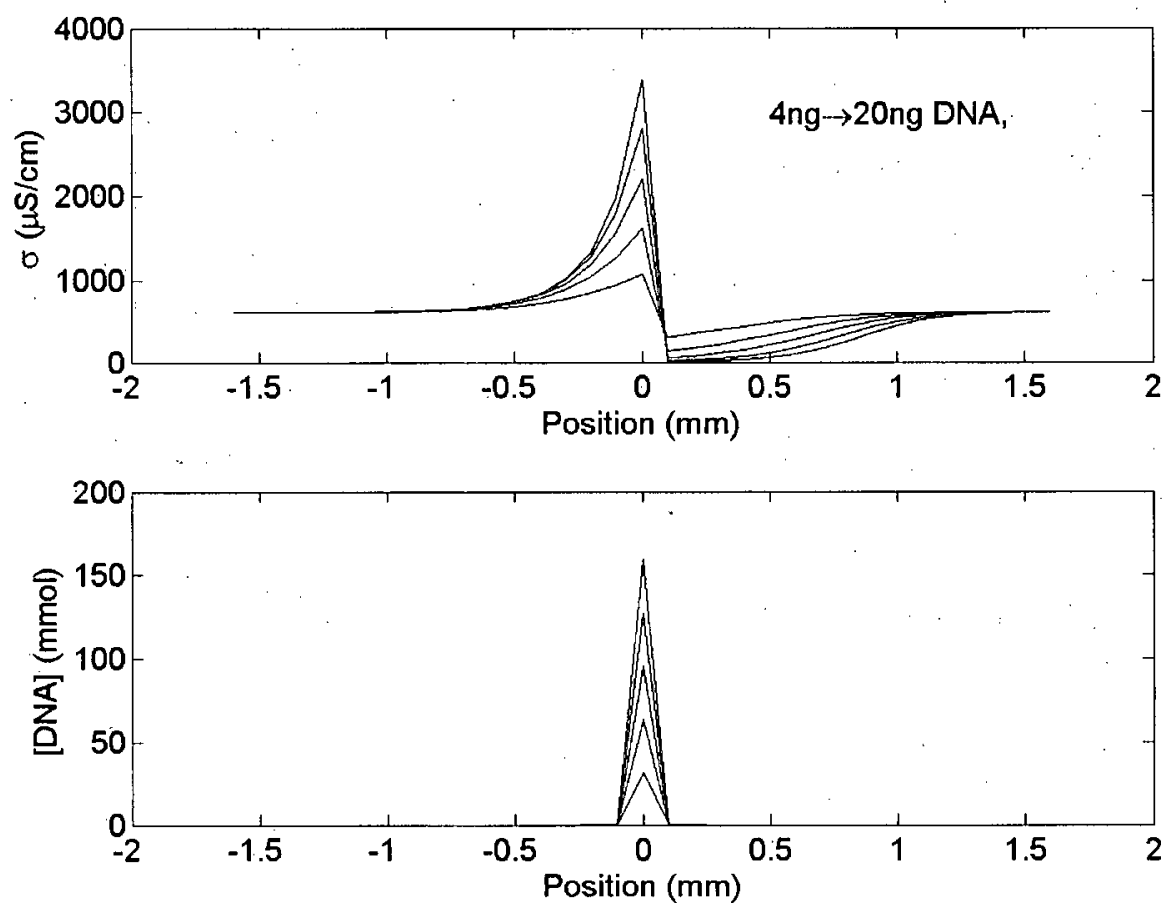

Figure 20 Effect of a fixed DNA peak on the background electrolyte conductivity. Near total depletion $(\sigma \sim 5 \mu \mathrm{S} / \mathrm{cm})$ is achieved after $20 \mathrm{~s}$ run time with $16 \mathrm{ng}$ of DNA

Figure 20 shows the effect of a series of fixed Gaussian DNA peaks of varying height on the background electrolyte after 20 seconds. The DNA peaks have a width of $200 \mu \mathrm{m}$, typical of those observed (eg Figure 13). As expected, the ion distribution shows a more extreme analog to the perturbation approximation in equation (5-10). The simulation was run under the same conditions as those used for the data in Figure 12, and as in Figure 12, near total depletion occurs with between 10 and $20 \mathrm{ng}$ of DNA present, in good agreement with experiment. Figure 21 shows the effect on the ion profile when the peak is broadened. The degree of depletion is independent of the width of the DNA peak. This agrees with equation (5-10) and explains the experimental observation that more conductive samples which 
exhibit a lesser degree of sample stacking have the same depletion behaviour as less conductive, and consequently more compressed, samples.
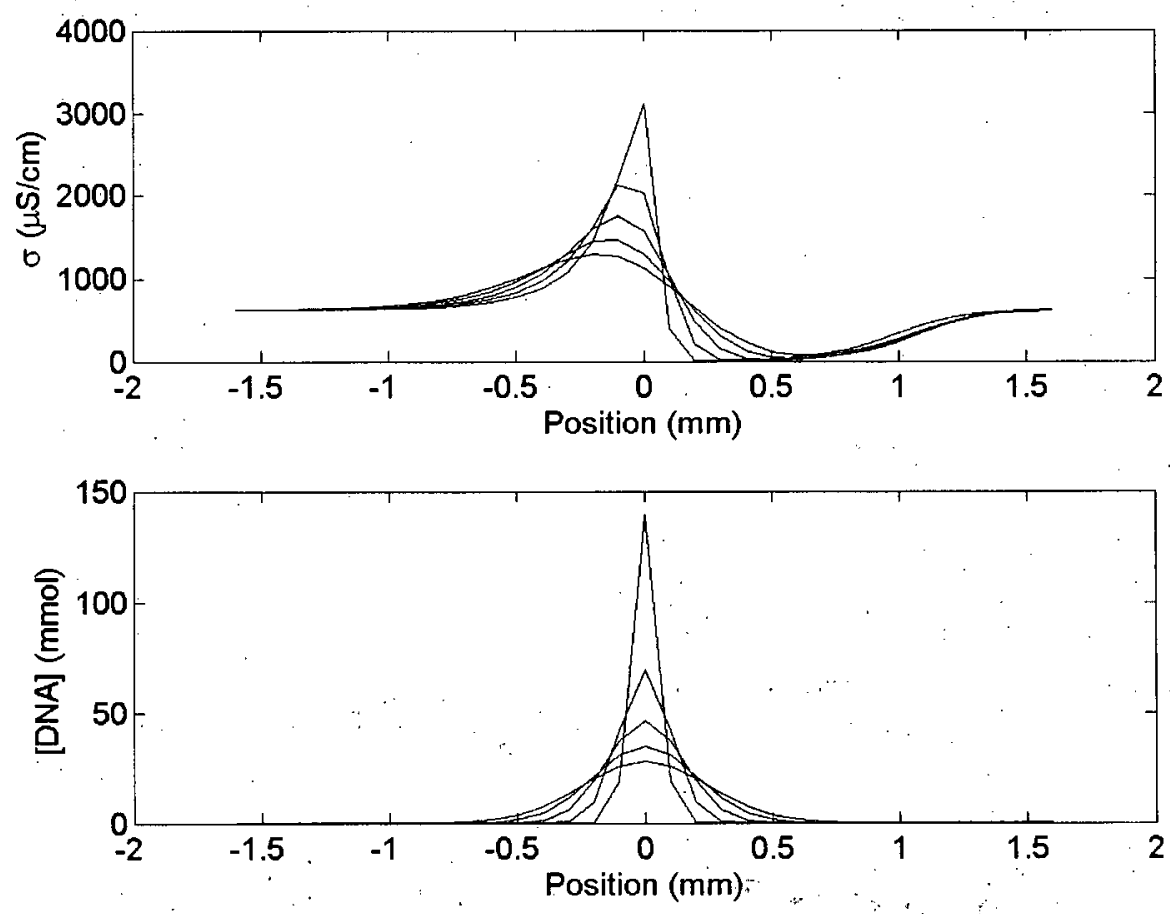

Figure 21 The effect of a $20 \mathrm{ng}$ DNA peak of varying width, after $20 \mathrm{~s}$. The degree of depletion is the same for each width.

Finally Figure 22, shows how reducing the mobility of the DNA deepens the depletion region. This simulation was again run in the frame of reference of the DNA so its mobility was subtracted from the TAPS ${ }^{-}$mobility and added to the $\mathrm{TrisH}^{+}$mobility. When the DNA mobility decreases, the TAPS- ion gets relatively faster, and the perturbation caused by the DNA peak gets more severe. 


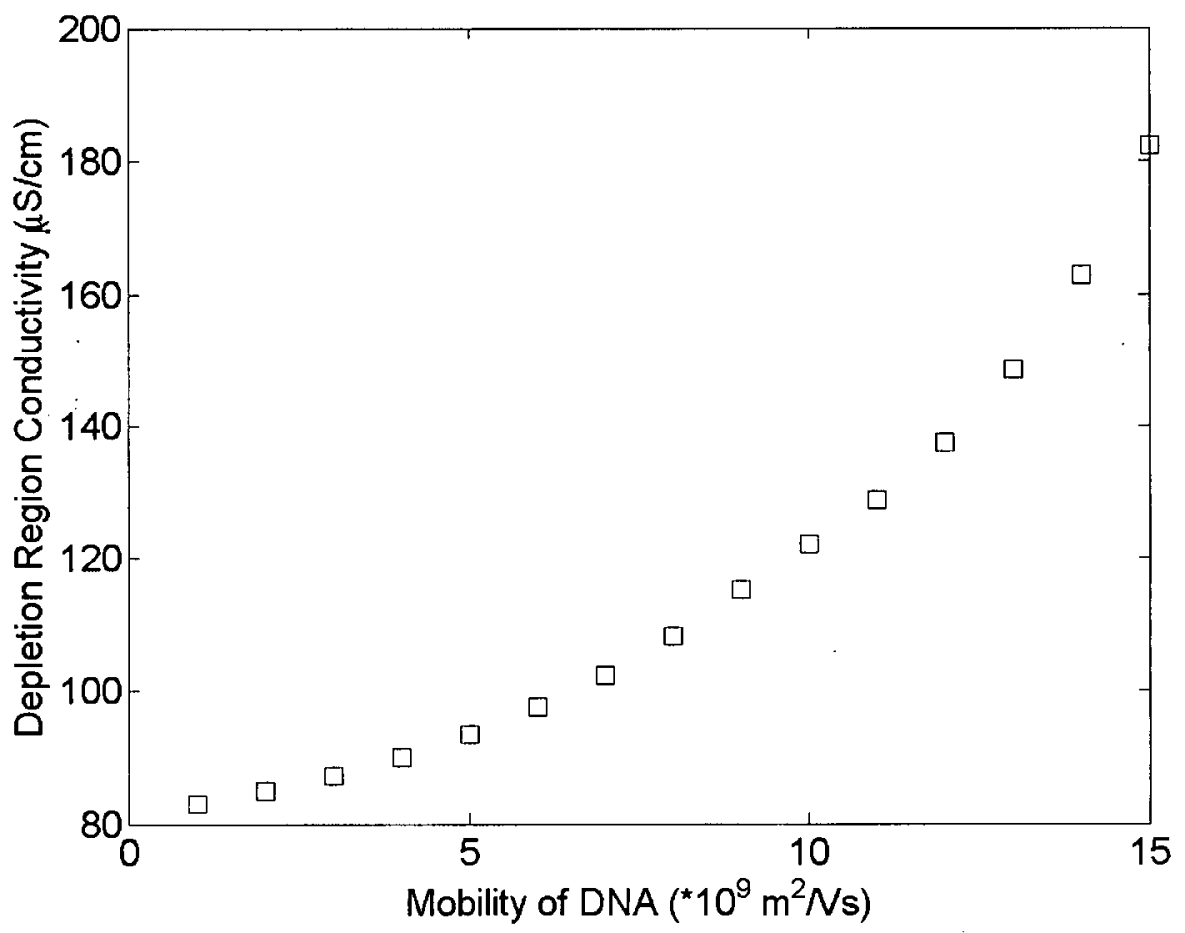

Figure 22 The effect of DNA mobility on depletion depth.

This simulation was performed in the frame of the DNA, with the DNA's nominal mobility subtracted from the TAPS' ion's and added to the TrisH $^{+}$mobility. $\lambda$ DNA in LPA has a mobility of $-2.5 \times 10^{-9} \mathrm{~m}^{2} / \mathrm{Vs}$. $18 \mathrm{ng}$ of DNA was used and the simulation was run for $20 \mathrm{~s}$.

\subsection{DNA and Cathode Boundary Propagation}

To understand why the depletion region boundaries propagate, we turn now to their movement in the frame of the capillaries. In section 4.4 it was noted that the anode boundary propagation velocity depends linearly on current while the cathode boundary does not. We also note that the presence of the DNA peak at the cathode boundary implicates the DNA mobility in the cathode boundary velocity, and note that the absence of DNA at the anode boundary points to imbalance in 
ion flow as the driving mechanisms for that boundary. The following discussion expands on these arguments.

Before considering a mechanism for cathode boundary movement, it should be considered whether, as in Figure 13 beyond the range of the IR camera, the anode boundary diverges from linearity as much as the cathode boundary. There is no reason to suppose from the IR images that this is not happening, as both boundaries appear linear over the first five minutes, and some variable EOF could in principle exist. To check this, four runs were conducted where DNA was injected, the run was thermally imaged and the capillaries were then cut up. The actual anode boundary position could then be compared with one extrapolated from the thermal image, where $x\left(t_{\text {final }}\right)$ $=C_{1} Q_{\text {final }}+C_{2}$. $C_{1}$ was determined by fitting to the anode boundary, and the offset $C_{2}$ was found by comparing the cut capillary and thermally imaged positions of the cathode boundary, which remained in view throughout. From this it was found that the difference between the actual and extrapolated anode positions was no more than $1.3 \%$ for the runs of 5,6 and 13 minutes and $10 \%$ for a run of 41 minutes. In the 41-minute run the cathode boundary, by comparison, lagged its linearly extrapolated position by $50 \%$. We conclude therefore that the anode boundary maintains its linearity of position with respect to charge throughout the run while the cathode boundary diverges from linearity. EOF, if it is present, must therefore remain relatively constant.

Given the hypothesis that the cathode boundary is driven by the propagating DNA, the nonlinearity of the boundary movement must be intrinsic to the DNA migration. It is known that in agarose, the mobility of long fragments has a field dependent term [75]

$$
v=\mu_{o} E+\mu_{1} E^{2} \text {. }
$$


Using the present system of fitting position against charge, this would take the form of

$$
x(t)=\frac{\mu_{o}}{\sigma A} \int_{0}^{t} I(\tau) d \tau+\frac{\mu_{1}}{\sigma^{2} A^{2}} \int_{0}^{t} I(\tau)^{2} d \tau .
$$

Such a function can only be fitted to the observed cathode boundary data if the coefficient $\mu_{0}$ is negative, i.e. the DNA is positive, which doesn't agree with any electrophoresis mechanism hitherto reported.

There is evidence however that at low fields, large fragments, which normally propagate under the biased reptation regime, fall into an entropic trapping regime where mobility is reduced [76]. The DNA is trapped in larger pores in the matrix, but within the influence of the force field generated by the applied voltage, thermal energy imparted to the DNA allows it to migrate through the constrictions to the next trapping site. This is modeled as an Arrhenius process of energy barrier crossing between trapped states, with negligible probability of a reverse reaction (i.e. backward migration induced by thermal motion), and is characterized by a Boltzmann factor that includes the energy barrier height associated with crossing between trapped states, together with the applied potential:

$$
v=v_{0} e^{\left(Q x E-U_{0}\right) / k T}
$$

Here, $E$ is the local field and $Q x$ is a parameter to be found that combines the effective number of elementary charges available to move the DNA despite screening, multiplied by the length scale of the energy barrier.

At higher fields $\left(E>E_{0}=U_{0} / Q x\right)$, enough force is applied to the DNA to remove the barrier entirely, and migration is constant. In this regime, normal electrophoretic behaviour characterized by (5-25), is expected to apply. To accommodate the threshold behaviour between the two regimes, the sigmoid function is used 


$$
Y=\frac{1}{\left(1+e^{-\left(x-x_{t}\right) / x_{o}}\right)}
$$

where $x_{t}$ is the threshold and $x_{0}$ is a measure of the steepness of the transition from 0 to 1 . Combining (5-25) and (5-27) with the sigmoid function, and putting all driving parameters in terms of currents gives

$$
v=\left(1-\frac{1}{1+e^{-\left(I-I_{0}\right) / x_{o}}}\right) \nu_{0} e^{\left(I-I_{0}\right) / I_{c}}+\left(\frac{1}{1+e^{-\left(I-I_{0}\right) / x_{o}}}\right)\left(\mu_{0} I+\mu_{1} I^{2}\right)
$$

This is a 6 parameter fit: $\mu_{0}, \mu_{1}$; are the two mobility terms $I_{0}$, the threshold for trapping; $I_{c}$ which is related to the effective charge of the $\lambda$ DNA and the trap escape distance, and temperature; $v_{0}$, related to the relaxation time of $\lambda$ DNA in LPA; and $x_{0}$, the sigmoid steepness parameter. Here we set the steepness parameter $x_{0}$ to 0.1 so that the fit requires five parameters. In effect though, this is two separate fits of two parameters, one in the low field and one in the high field regime, with a threshold value to be found between them. 


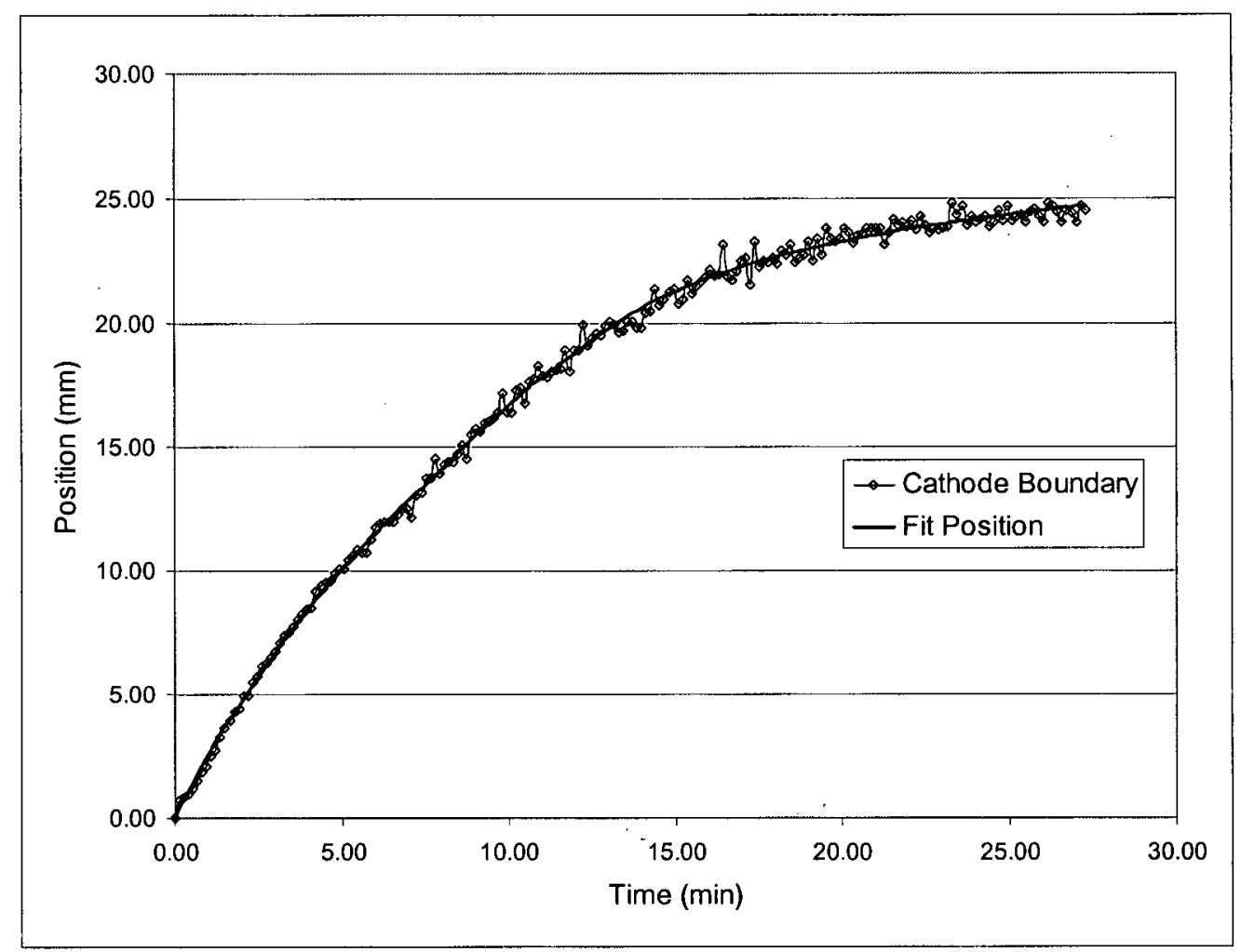

Figure 23 Fit of equation (5-29) to a cathode boundary position.

Parameters at $293 \mathrm{~K}$ are $\mathrm{U}_{0}=6 \mathrm{kT}, \mu_{0}=1 \times 10^{-9} \mathrm{~m}^{2} \mathrm{Ns}, \mu_{1}=1.2 \times 10^{-13}$ $\mathrm{m}^{3} / \mathrm{V}^{2} \mathrm{~s}, \mathrm{v}_{0}=2 \times 10^{-2} \mathrm{~mm} / \mathrm{s}, Q \times / \mathrm{k} T=8 \times 10^{-4} \mathrm{~m} / \mathrm{V}$.

Viewed independently, the parameters of the fit for the two regimes are quite reasonable. In the biased reptation regime, the linear term $\mu_{0}=$ $1 \times 10^{-9} \mathrm{~m}^{2} \mathrm{Ns}$ is close to measured values of $\mu$ for $\lambda$ DNA in the absence of depletion regions. It is also very close to the value for agarose reported in [75] although the nonlinear term in this case is an order of magnitude smaller.

The entropic barrier $U_{0}=E_{0} Q x$ can be overcome when the field is strong enough that the electric forces are comparable to the thermal forces acting on the DNA. This occurs when $E_{0}=k T / q b$ [77], where $b \sim 100 \mathrm{~nm}$ is the Kuhn length (twice the persistence length) for dsDNA, and $q$ is the effective charges per Kuhn length - about $20 e^{-}$for dsDNA [78]. Q, the number of charges available to move the DNA, is q multiplied by the number 
of Kuhn lengths $n_{k} \sim 150$ for $\lambda$ DNA. The length parameter $x$ can be taken as the pore size of the matrix, which is $\sim 3 \mathrm{~nm}[20]$. This gives an expression for $U_{0}$ :

$$
\frac{U_{o}}{k T}=\frac{n_{k} q x}{q b} .
$$

Using the numbers for $n_{k}, x$ and $b$, gives Uo $\sim 6 \mathrm{kT}$, as was found in the fit. $Q x / k T$ itself can be estimated from the data above as

$$
\frac{Q x}{k T}=\frac{n_{k} q x}{k T}
$$

which gives an estimated value of $Q x / k T=4 \times 10^{-4} \mathrm{~m} / \mathrm{N}$, compared to $8 \times 10^{-4} \mathrm{~m} / \mathrm{V}$ from the fit. The parameter $v_{o}$ is the velocity of DNA as it falls into the entropic regime. In this case the midpoint of the sigmoid is found to be at $2.24 \mu \mathrm{A}$, which, if $(5-25)$ is used, also gives $.02 \mathrm{~mm} / \mathrm{s}$. This makes intuitive sense as the velocities in the two regimes must match at the transition point.

No experimental confirmation of this effect in polymer matrices has been found in the literature, but the absence of such data was noted by Jean Louis Viovy in 2000 : it is somewhat surprising that it [entropic trapping] has been overlooked for quite a long time in the context of gel electrophoresis. This is probably due to the conditions necessary for its appearance, and in particular the requirement of low fields: such conditions are not very interesting as far as separation is concerned, and they were avoided by experimentalists on a pragmatic basis. [76]

We have shown here that the behaviour of DNA in the entropic trapping regime may in fact be interesting, but from a high-throughput, low-cost DNA sequencing perspective, it would be generally desirable if entropic trapping of large fragments, and in fact, large fragments themselves, were entirely avoided! 


\subsection{Anode-Side Boundary Movement}

Experimental results show that anode-side boundaries in both the DNA-induced and depleted buffer cases are not associated with the presence of DNA. The ions at the anode side boundary must therefore be the background Tris and TAPS ions as well as $\mathrm{H}^{+}$and $\mathrm{OH}^{-}$ ions. There could conceivably be other ions from the buffer or matrix present, but their behaviour should in principle be typical of small ions and therefore predictable. A configuration and behaviour of these ions on both sides of the boundary must then be found which can explain the boundary movement. In order to do this, it is best to start with the basic theory of moving boundaries in systems of multiple ions. The proposed models for anode boundary propagation can then be best understood in the context of the failure of this theory in describing the observed propagation

\subsubsection{The Theory of Moving Boundaries}

The behaviour of ionic boundaries has been the subject of research for over a century [79]. There are several types of ionic boundaries, including those between two strong electrolytes sharing a common counterion, weak electrolytes where $\mathrm{H}^{+}$and $\mathrm{OH}^{-}$may play a role, and concentration boundaries with the same positive and negative ions on both sides but at different concentrations. We begin by examining the simplest system: two different ion populations in adjacent regions, with a common counterion [80]. For thematic continuity, we shall assume the adjacent ions are negative and the common counterion is positive. Consider a system where ion 2 , with concentration $C_{2}$ moves with velocity $v_{2}$ into a region which is being vacated by ion 1 , of concentration $C_{1}$ and velocity $v_{1}$ as shown in Figure 24. We also make the assumption for now that $\mu_{1}>\mu_{2}$. The counterion, 
of course, has concentrations $C_{1}$ and $C_{2}$ to balance the negative ions everywhere, and a mobility $\mu_{+}$.

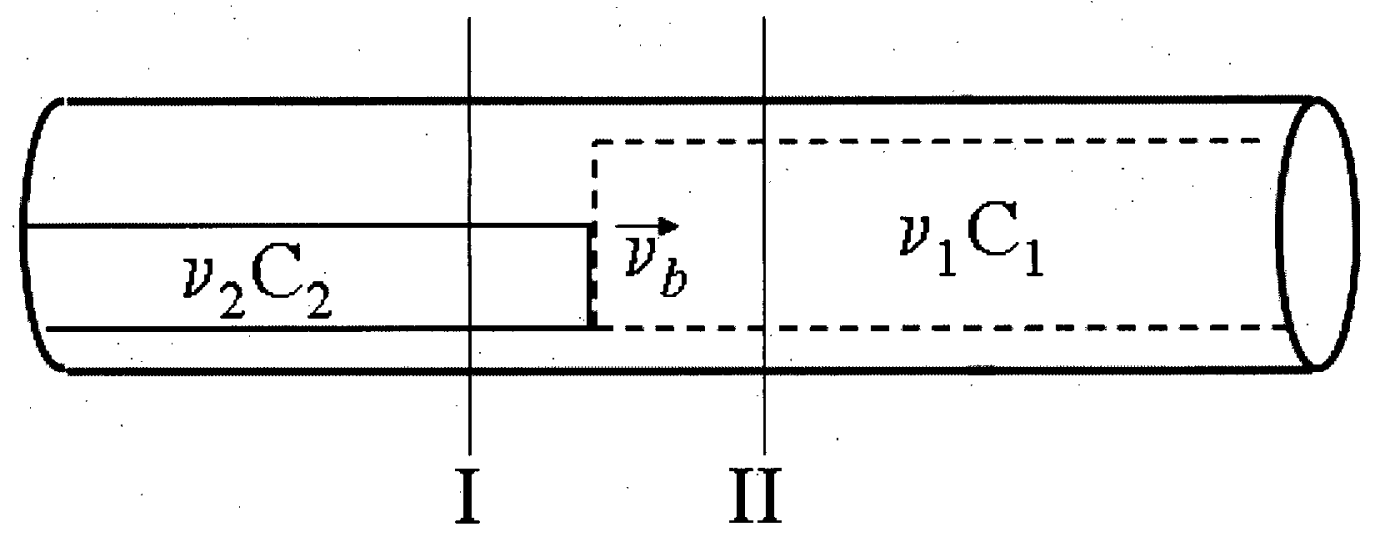

Figure 24 Adjacent region of ion 1 (dashed lines) and 2 (solid lines) forming a moving boundary with velocity $v_{b}$.

It will be shown below that conservation of ion flux requires certain values of $\mathrm{C}_{2}$ in order for the boundary to move but we assume for the moment that this moving boundary satisfies that condition. The change in concentration over time between boundaries I and II is equal to the difference in flux across the two boundaries and also equal to the difference in concentration multiplied by the movement of the boundary swept out between them.

$$
\Delta \Theta=v_{2} C_{2}-v_{1} C_{1}=v_{b} C_{2}-v_{b} C_{1} .
$$

Solving for $v_{b}$ and given $\nu=\mu E=\mu J / \sigma$ we get

$$
v_{b}=\frac{J}{F}\left(\frac{\mu_{2}^{-}}{\mu_{2}^{-}+\mu^{+}}-\frac{\mu_{1}^{-}}{\mu_{1}^{-}+\mu^{+}}\right)\left(\frac{1}{C_{2}-C_{1}}\right) .
$$

The mobilities can be rewritten as

$$
T_{n}=\frac{\mu_{n}^{-}}{\mu_{n}^{-}+\mu^{+}} .
$$

$T_{n}$ is known as the transference number and represents the relative conductivity of the ion and counterion. Then $v_{b}$ becomes 


$$
v_{b}=\frac{J}{F} \frac{T_{2}-T_{1}}{C_{2}-C_{1}} .
$$

Conservation of charge flux everywhere in the capillary requires that only certain values of $C_{2}$ are allowed in (5-35). This was first pointed out by Friedrich Kohlrausch in 1897 [79]. Assuming for the time being that the mobilities are constant with respect to ionic concentration, we write them as $\mu_{i}=z_{i}\left|\mu_{i}\right|$ where $z_{i}$ is the charge of the ions. Neglecting diffusion, the sum over all the ions gives:

$$
\sum_{i} \frac{1}{\left|\mu_{i}\right|} \frac{\partial C_{i}(x, t)}{\partial t}=\frac{\partial}{\partial x}\left(E(x, t) \sum_{i} z_{i} C_{i}(x, t)\right)
$$

but charge neutrality requires that everywhere

$$
\sum_{i} z_{i} C_{i}(x, t)=0
$$

thus

$$
\sum_{i} \frac{1}{\left|\mu_{i}\right|} \frac{\partial C_{i}(x, t)}{\partial t}=0
$$

and integrating gives

$$
\sum_{i} \frac{C_{i}\left(x, t_{o}\right)}{\left|\mu_{i}\right|}=\omega(x)
$$

Equation (5-39) implies that after initial conditions are established by, say, hydrostatically injecting some combination of electrolytes into the capillary, the value of $\omega(x)$ remains constant in time. This equation is known as the Kohlrausch Regulating Function, and once electrophoresis starts, it determines the concentrations of species that follow moving boundaries between species. Equation (5-39) requires that as ion 2 moves into the region vacated by ion 1 in Figure 24, it has concentration 


$$
C_{2}=C_{1}\left(\frac{\mu_{1}}{\mu_{2}}\right)\left(\frac{\mu_{2}+\mu_{+}}{\mu_{1}+\mu_{+}}\right)
$$

Applying (5-40) to (5-33) gives, after some simplification,

$$
v_{b}=\frac{J}{F C_{1}} \frac{\mu_{1}}{\mu_{1}+\mu_{+}}=\mu_{1} E_{1},
$$

so the boundary moves at the speed of the leading ion. Measuring such a boundary between strong electrolytes of different mobilities and combining and obtaining conductivity data for the leading solution turns out to be the best way to measure the transference number (5-34) of the leading electrolyte at different ion concentrations [80]

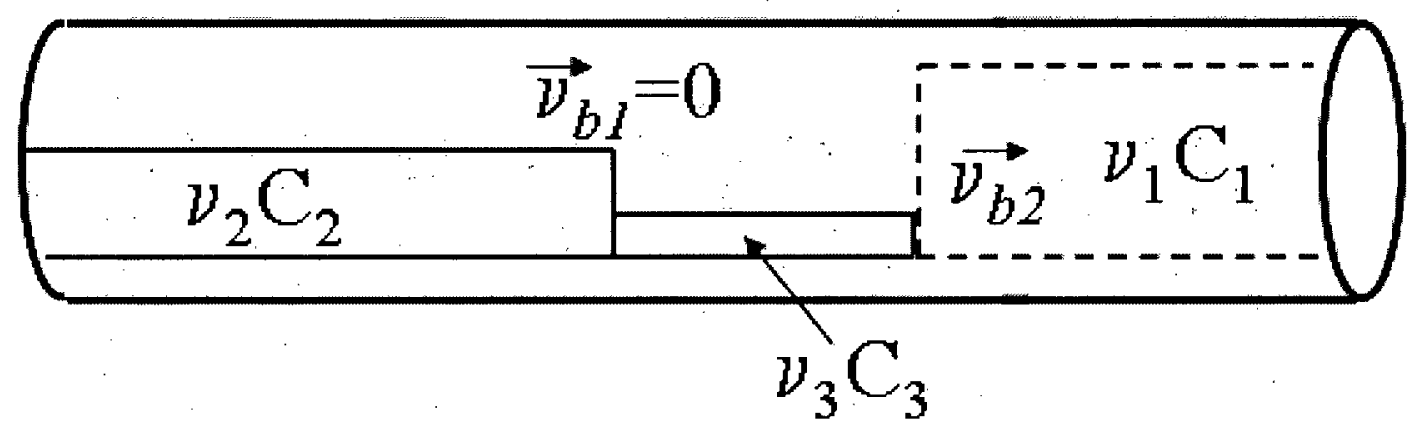

Figure 25. The effect of the regulating function on the distribution of ions in the capillary. Initial conditions are as in Figure $\mathbf{2 4}$ and ion 1 (dashed lines) is leading ion 2 (solid lines). The regulating function (5-39) requires that Ion 2 takes on concentration $C_{3}$ to conserve total ion flux. The new concentration boundary in ion 2 remains fixed.

The actual picture for strong electrolytes is shown in Figure 25, which represents what would happen if the system were initialized at $t$ $=0$ as shown in Figure 24 and then run for some time. The regulating function automatically adjusts the concentration of the region following ion 1 giving a new concentration for ion 2 of $C_{3}$. This then gives the 
correct velocity of the trailing ions to match the leading ion. The new boundary $v_{b 1}$ between regions of different concentrations of ion 2 however, should be stationary. These three regions represent different "zones", hence the general term "capillary zone electrophoresis" in common use in analytical chemistry.
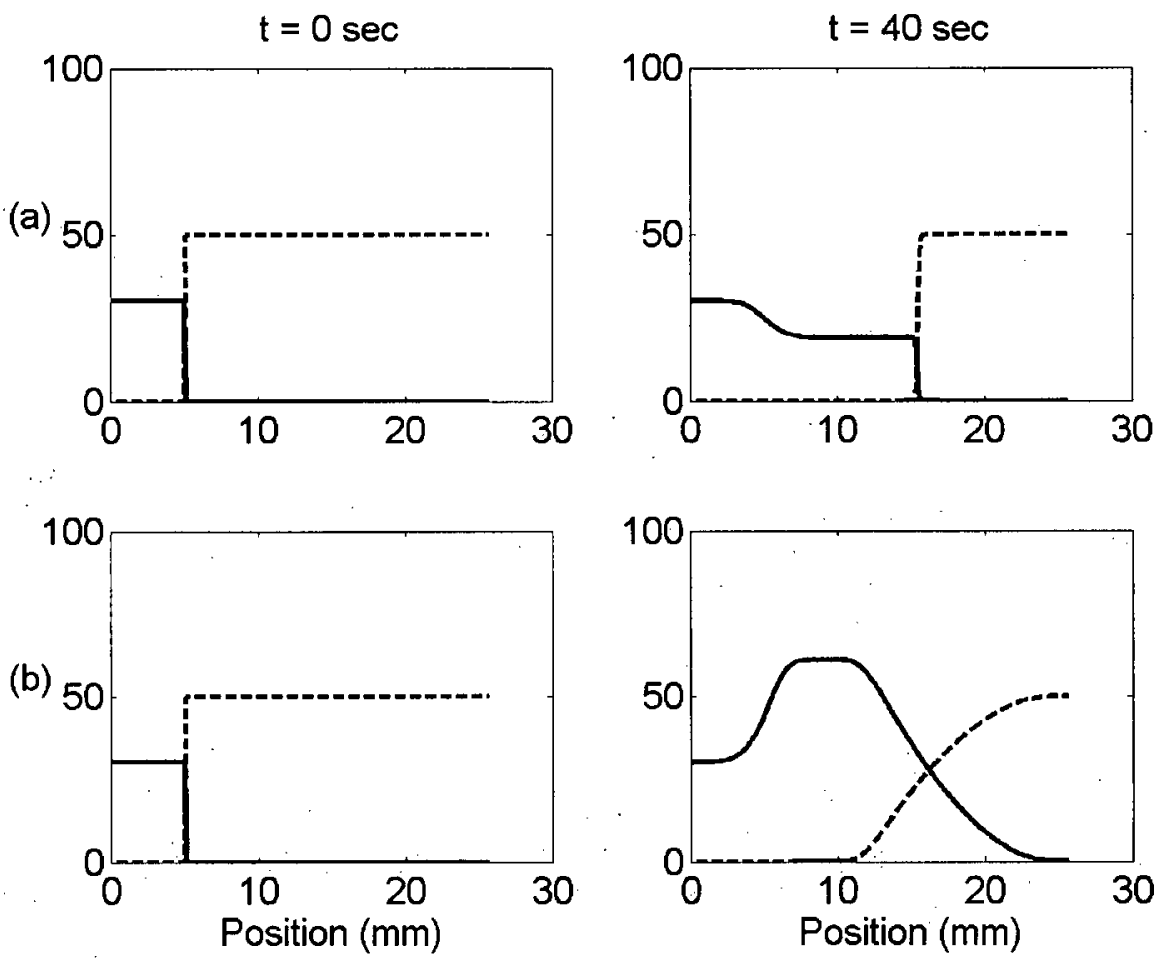

Figure 26 Simulation of a leading (dashed line, $\mu=\mu_{1}$ ) and trailing (solid line, $\mu=\mu_{\mathrm{t}}$ ) negative ion. Initial states are at left and, after $40 \mathrm{sec}$, at right. (a) $\mu_{t}<\mu_{l}$ so the moving boundary remains sharp, (b) $\mu_{t}>\mu_{l}$ so moving boundary smears out.

Simulations of two strong electrolytes with a common counterion and varying mobilities are shown in Figure 26 . In (a) the leading ion has a higher mobility than the trailing ion and in (b) the trailing ion has a higher mobility. The adjustment of the trailing ion concentration to conserve charge flux can be observed. The mobile and stationary 
boundaries are visible, though the fixed boundary at the original interface location is subject to numerical diffusion.

If the classical moving boundary model were to apply, there would have to be another negative ion following the TAPS $^{-}$in the depletion region. While there is no evidence for such a hypothetical ion, it can also be shown that the physical properties required by theory make it unlikely that such an ion could exist. Consider the depletion region as region 3 in Figure 25 page 89 . For such a boundary to be self sharpening, the ion in the depletion region must have lower mobility than the leading TAPS ions' mobility of $25 \times 10^{-9} \mathrm{~m}^{2} / \mathrm{Ns}$. In that case the boundary would propagate at the speed of the TAPS ion, but in reality the boundary actually propagates at, at most, one fifth that speed. The regulating function can be used to solve for the mobility of the hypothetical ion, given the experimentally measured concentrations on either side of the boundary. This gives a mobility of $4 \times 10^{-9} \mathrm{~m}^{2} \mathrm{Ns}$, which is much lower than any ionic species save very large DNA fragments. The only species present which might be negatively charged and might have such a mobility would be fragments of acrylamide polymers, but conservation of mass would prevent any bulk flow of such ions so this is unlikely.

\subsubsection{Common Ion Boundaries}

Previous work on anomalous conductivity in electrophoresis [43, $62,65]$ has cited Michael Spencer's three 1983 papers on the subject [81] [82] [83] as providing a possible explanation for current decline. Building on the prewar ion boundary work of Longsworth and others [80], Spencer argued that a change in transference number from differential retardation of ions could induce a depletion region at the gel buffer boundary. If the transference number was dependent on ion concentration, the newly formed boundary could then propagate into 
the matrix. The first part of this theory has been experimentally verified by Swerdlow [42] and Figeys [43] et al. as noted in section 2.5, page 16. It has not, however, been shown that a transference number change alone is responsible for boundary movement into the matrix without an accompanying $\mathrm{pH}$ change or involvement of another ion species. Analytic arguments and simulations are presented here which suggest that this boundary movement is unlikely to come from a concentration dependent transference number in general, and particularly not in the present case with Tris/TAPS buffer.

The extant literature on these "common-ion" boundaries consists of two papers. Smith, in 1931 [84], reproduced the correct magnitude and direction of a $200 / 100 \mathrm{mmol} / \mathrm{L} \mathrm{LiCl}$ boundary, which has a notably large change in transference number, though did not report boundary motion for other species with a smaller $d T / d C$ values. Spencer's own experimental results [83] show boundaries accompanied by $\mathrm{pH}$ changes. Prediction of the direction of boundary propagation based on the transference number gave the right direction, but more quantitative agreement could not be shown. Spencer also shows how the $\mathrm{pH}$ would change with depletion for a typical single ion weak electrolyte, but no prediction of boundary velocity is given in the case where water ions become significant carriers [82]. Elsewhere, Chien [85] suggests common-ion boundaries are stationary to at least first order, and Beckers and Bocek [86] assert that the only known moving common-ion boundaries are accompanied by a change in $\mathrm{pH}$, or are multivalent ions near pKa.

Spencer's prediction for common-ion boundary movement was tested via simulation. Spencer derived an expression for boundary movement dependent on a change in relative mobility (transference number) of two ionic species with concentration dependent mobilities. 


$$
v_{b}=\frac{J}{F} \frac{d T}{d C}
$$

If $d T / d C \neq 0$, the boundary can move and will be self-sharpening, as the point of lowest concentration has the highest velocity. The simulation was done for both buffered and unbuffered systems. $d T / d C$ $\neq 0$ is satisfied if the mobility of the carrier ions vary with concentration by different amounts. A common empirical formula for $\mu(C)$ for strong electrolytes such as $\mathrm{KCl}$ and $\mathrm{NaCl}$ in the range of $1-100 \mathrm{mmol} / \mathrm{L}$ is given in (5-43) where the coefficient 0.5 is found to be typical for univalent ions [87]. Assuming that the constant would be slightly different for different ions, we arbitrarily assign

$$
\begin{aligned}
& \mu_{+}=\mu_{+}^{0} \exp \left(-0.5 \sqrt{C_{+}}\right) \\
& \mu_{-}=\mu_{-}^{0} \exp \left(-0.6 \sqrt{C_{-}}\right)
\end{aligned}
$$

These are for the principal carrier ions. We assume that in the buffered system, the water ions have their usual mobilities. These are arbitrary ions, but for the sake of comparison, their initial mobilities are the same as the Tris and TAPS ions. 

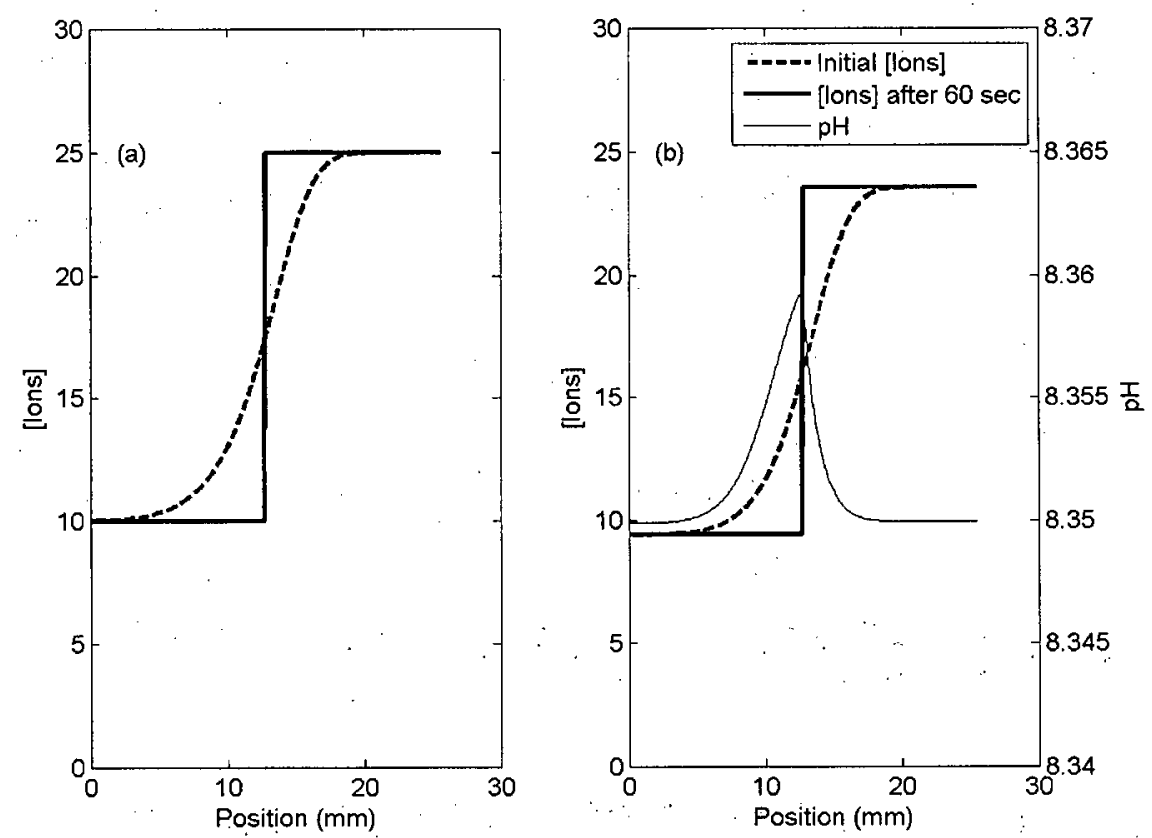

Figure 27 (a) Initial (solid line) and final (dashed line) ion distributions for two unbuffered ions over 60 seconds. (b) The same simulation in a buffered electrolyte, showing a change in $\mathrm{pH}$ near the boundary.

Figure 27 shows the results of the simulation for two and four ions. The boundaries show numerical diffusion and in both cases the center of the final concentration profile moves to the right at a rate of $\sim 0.5 \mathrm{~m} / \mathrm{C}$. The boundaries move the same direction irrespective of the sign of $\mathrm{dT} / \mathrm{dC}$ however, so the observed movement is likely an artifact of the numerical diffusion. When water ions are included there is a small change in $\mathrm{pH}$, as shown, but the same $\mathrm{pH}$ change occurs for both variable and constant ion mobility. Numerical diffusion appears to dominate any actual movement of the boundary, and may in fact obscure changes in boundary movement due to nonzero $\mathrm{dT} / \mathrm{dC}$. The model cannot, therefore, give a definitive answer to the question of whether such a like-ion boundary moves, but certainly does not 
reproduce observed propagation rates even with large changes in transference number.

The possibility of the observed boundaries propagating because of changes in transference number appears equally unlikely when considered analytically. The fastest moving boundaries we see in normal matrix propagate at $10 \mathrm{~m} / \mathrm{C}$. The conductivity falls from 1100 $\mu \mathrm{S} / \mathrm{cm}$ in the background to $70 \mu \mathrm{S} / \mathrm{cm}$ in the depletion region. Absent a pH change, the concentration of Tris $^{+}$and TAPS- would be $3 \mathrm{mmol} / \mathrm{L}$ in the depletion region compared to $50 \mathrm{mmol} / \mathrm{L}$ in the background. If this were a common-ion moving boundary, equation (5-42) would require a change in transference number of $20 \%$ to give $10 \mathrm{~m} / \mathrm{C}$. This seems implausible as typical transference number changes are more like $5 \%$ over $1-100 \mathrm{mmol} / \mathrm{L}$ [87] [88] for strong electrolytes. These are accompanied by changes in molar conductivity (conductivity/concentration) of $20 \%$. Figure 47 , page 157, of $\sigma$ vs. [Tris T.APS] in fact shows no measurable change in molar conductivity. at all, so it is very unlikely that changes in transference number can explain this boundary. Finally, the clipped capillary data shown in C.1 shows that it is definitely possible for a concentration boundary to exist in the capillary, as seen by thermal imaging, but which remains stationary because its upstream driving source has been removed. It must be concluded therefore, it is not the case that these boundaries move because of changes in relative conductivity brought on by concentration dependent mobilities, and that the explanation must lie in some system involving more than two-ions.

\subsubsection{Boundary Movement by Bound Charge}

There are two other possible mechanisms which, when simulated, produce behaviour similar to that observed experimentally. The first is that a change in $\mathrm{pH}$ across the boundary allows extra $\mathrm{OH}^{-}$ 
ions from the depletion region to neutralize acid species at the boundary, causing it to advance. The second is that some small quantity of bound charge may exist on the acrylamide or capillary surface which might produce a moving boundary. Simulations and analytical analysis of the $\mathrm{pH}$ mismatch model, shown below, show boundary propagation and depletion depths comparable to experiment. What is lacking however is a mechanism by which the $\mathrm{pH}$ may rise sufficiently to explain the observed boundary movement. The bound charge model, which also produces $\mathrm{pH}$ changes, does have a realistic source so we focus on this model first.

There is ample evidence from the literature that polyacyliamide can become charged over time as the monomers become hydrolyzed. $[89,90]$. Chiari et al,for example, found more that $30 \%$ of polyacrylamide monomers became hydrolyzed in 0.1 Molar $\mathrm{NaOH}$ in less than an hour. [91]. While the present LPA is at a much lower $\mathrm{pH}$, it is reasonable to assume that it has some small degree of bound negative charge, Given that $4 \%$ w/v LPA has approximately $500 \mathrm{mmol} / \mathrm{L}$ acrylic monomers, we will make the assumption that of the order of $1 \mathrm{mmol} / \mathrm{L}$ of the chain components are charged. It is also possible that the LPA might become charged only when the electrolyte is depleted. This would be quite possible if the acrylamide were behaving as a weak acid, but this behaviour has not been reported in the literature and will again require a $\mathrm{pH}$ change.

A velocity for a boundary driven by fixed charge can be derived in similar manner to the derivation in section 5.4.1. Without bound charge, such a like-ion concentration boundary should not move. The bound charge introduces a change in the proportion of the positive and negative mobile species however, so effectively the transference number changes. Consider again the ion-plus-counterion situation in Figure 24 , page 87 . Here, we assume a constant concentration $C_{f}$ of 
bound negative charges throughout the system. The ions have a constant mobility and we assume for simplicity that $\mu_{-}=\mu_{+}$. The negative ion has concentrations $C_{1}$ and $C_{2}$ but the bound negative charges give the positive ions concentrations $C_{1}+C_{f}$ and $C_{2}+C_{f}$. The flux of negative ions in the two regions is again given as

$$
\Delta \Theta=v_{2} C_{2}-v_{1} C_{1}=v_{b} C_{2}-v_{b} C_{1} .
$$

now however, the velocity becomes

$$
v_{b}=\frac{J}{F}\left(\frac{\mu C_{2}}{\mu C_{2}+\mu\left(C_{2}+C_{f}\right)}-\frac{\mu C_{1}}{\mu C_{1}+\mu\left(C_{1}+C_{f}\right)}\right)\left(\frac{1}{C_{2}-C_{1}}\right)
$$

or

$$
v_{b}=\frac{J}{F}\left(\frac{C_{2}}{2 C_{2}+C_{f}}-\frac{C_{1}}{2 C_{1}+C_{f}}\right)\left(\frac{1}{C_{2}-C_{1}}\right) .
$$

Propagation rates for a range of bound charges and degrees of depletion is shown below in Figure 28. 


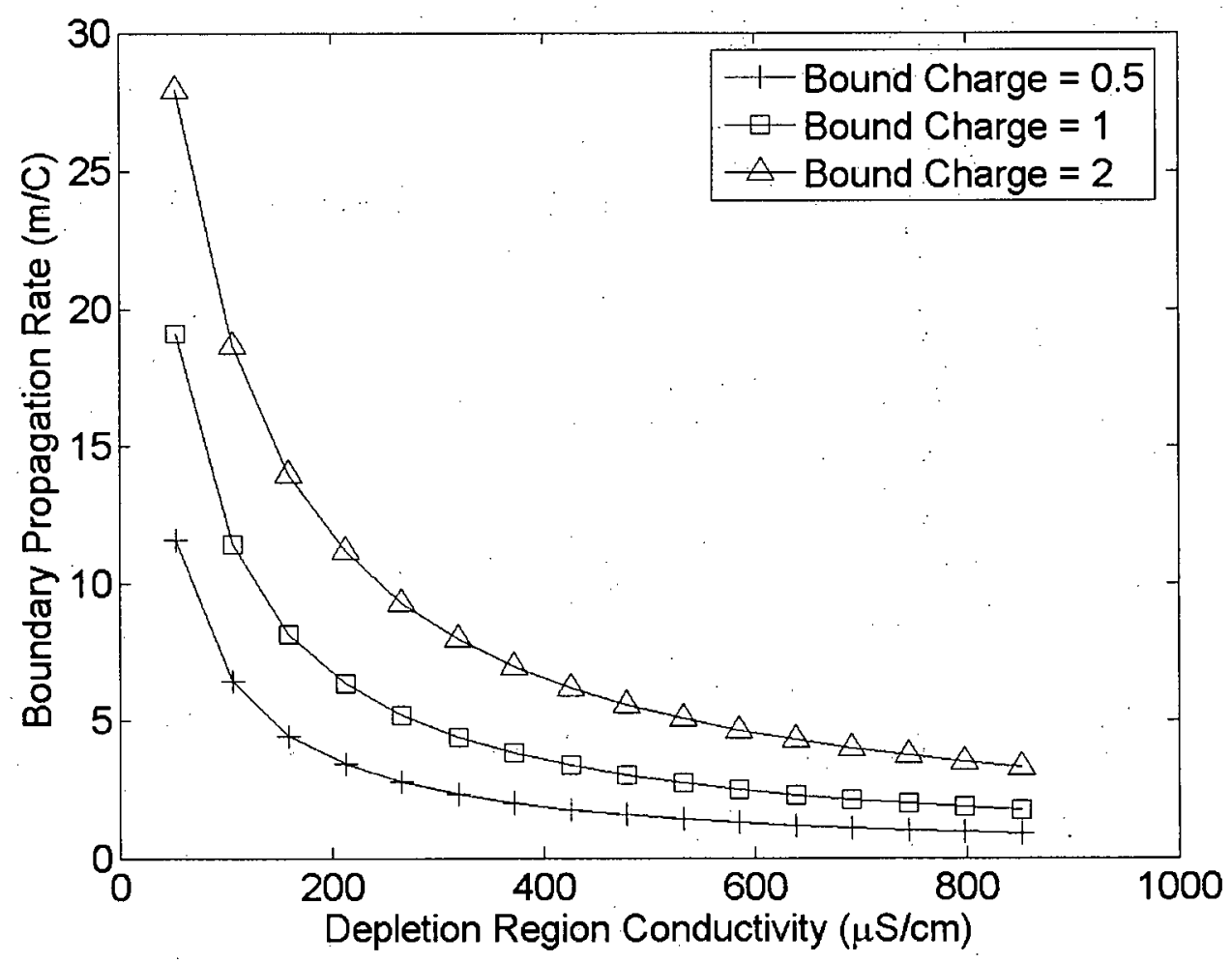

Figure 28: Plots of equation (5-45) for different densities of bound charge. The background conductivity was $950 \mu \mathrm{S} / \mathrm{cm}$.

Simulations were next performed with the full chemical equilibrium model plus bound charge. These bound negative charges were initialized simply by adding the equivalent concentration of $\mathrm{Tris}^{+}$ throughout the capillary. The concentration of buffer at the cathode end was then diluted by various fractions to give a range of conductivities typical of those observed experimentally in the depletion region. A comparison of these results with experiment is shown in Figure 29 below. 


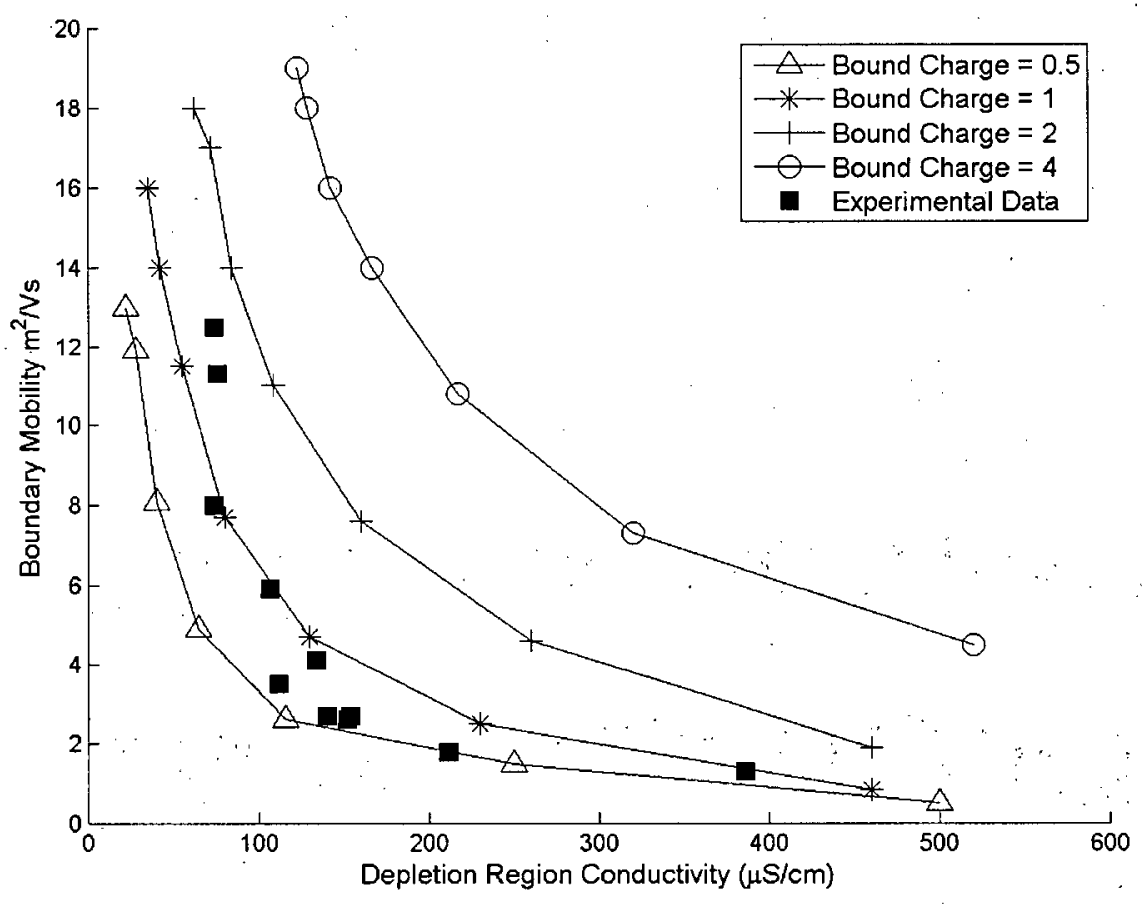

Figure 29 Experimental boundary propagation compared to simulation of boundary propagation due to bound charges. Bound charges are in $\mathrm{mmol} / \mathrm{L}$. Simulated cathode buffer concentrations are $(0.5,1,2,4,8,16) \mathrm{mmol} / \mathrm{L}$ and background electrolyte $=50 \mathrm{mmol} / \mathrm{L}$. Zero bound charge failed to produce a moving boundary

This model approximately reproduces the behaviour of the boundary observed experimentally. Unlike the $\mathrm{pH}$ variation model discussed below, the boundary movement arises naturally from the decline in carrier ions. An interesting piece of data from Chiari et al is that PDMA appears to hydriolize about 500 times more slowly than LPA [91]. This may explain why PDMA is less prone to current decline than LPA.

A curious feature of these simulations is that for bound charge up to $1 \mathrm{mmol} / \mathrm{L}$, the $\mathrm{pH}$ rises in the depletion region whereas for 2 $\mathrm{mmol} / \mathrm{L}$ and above, the $\mathrm{pH}$ falls. The comparison is shown in Figure 30 
below. The cause of this change is not understood but is likely to be related to the limited buffer capacity in the depletion region. If there are not sufficient positive ions to counter the negative bound charge, the charge may act as an acid and drive the $\mathrm{pH}$ down, whereas where there is excess basic buffer, the $\mathrm{pH}$ may rise. In section 5.5 we present evidence of what appears to be a sustained rise in $\mathrm{pH}$. The present simulations do not show a sustained rise in $\mathrm{pH}$ while such behaviour is displayed by boundaries driven by larger $\mathrm{pH}$ mismatches. The latter mechanism is described below and while it may not be at work in the present situation, a definitive answer may have to await a more precise measurement of $\mathrm{pH}$ changes in the capillary.
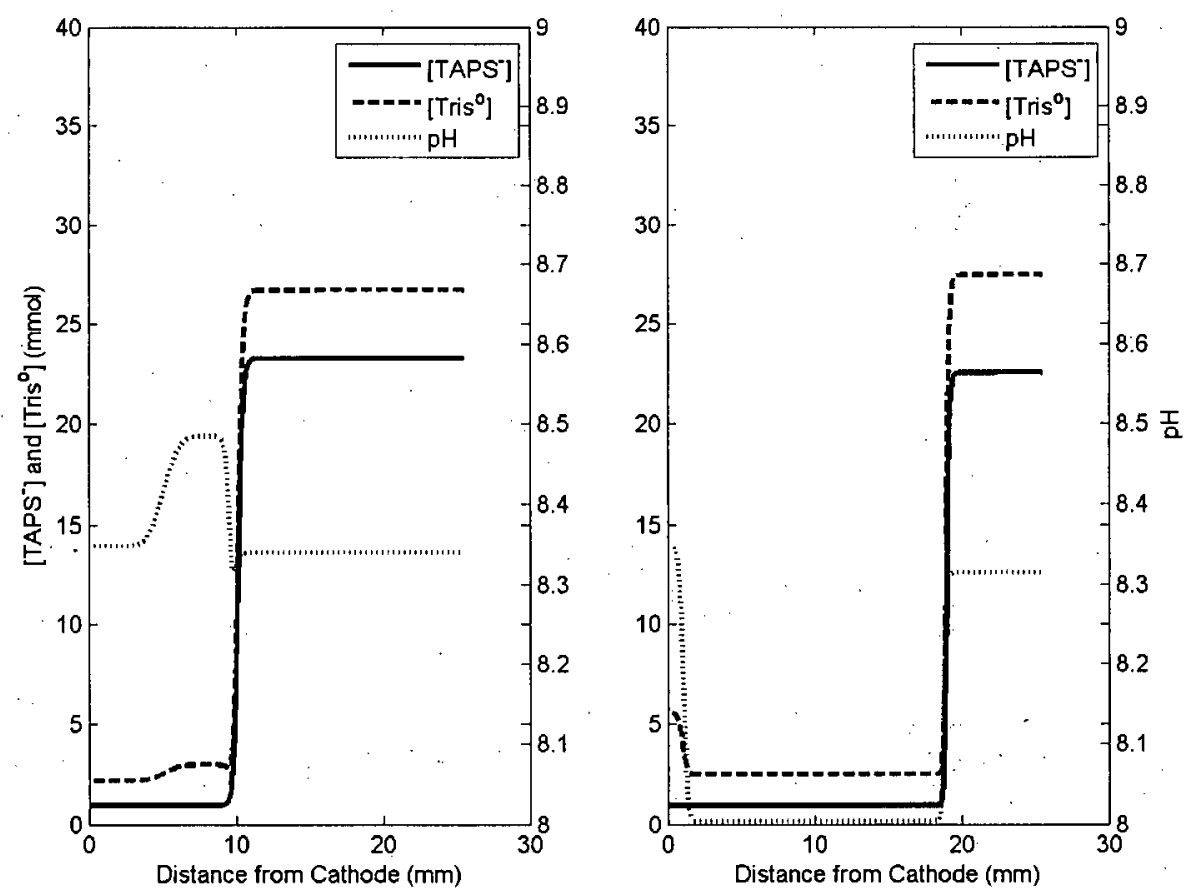

Figure 30 A comparison of behaviour between a bound charge of $1 \mathrm{mmol} / \mathrm{L}$ on the left and $4 \mathrm{mmol} / \mathrm{L}$ on the right. The variation in $\mathrm{pH}$ is apparent. Cathode buffer concentration and background electrolyte were $2 \mathrm{mmol} / \mathrm{L}$ and $50 \mathrm{mmol} / \mathrm{L}$ in both cases. 


\subsubsection{Experimental Evidence for $\mathrm{pH}$ Changes in the Depletion Region.}

The preceding simulations using bound charges reproduced the boundary movement, but do not suggest a substantial change in $\mathrm{pH}$. There are a couple of issues here. The first is that, as discussed in Appendix C.1, deep depletion regions can permanently damage the capillary surface coating so that depletion regions subsequently arise without special buffer conditions or DNA. Such an effect could arise from adsorbed DNA, a change in $\mathrm{pH}$, or the high fields associated with the depletion region. It is hard to understand how anything but a $\mathrm{pH}$ change could affect the chemical structures at the capillary surface. A high enough electric field would have to be strong enough to affect the physical structure of the matrix. Second, it is shown in this section that nickel particles seem to respond to the depletion region in a manner consistent with a sustained rise in $\mathrm{pH}$.

The following experiments were motivated by a desire to measure any residual EOF in capillaries. Such measurements are normally done with neutral EOF markers such as dimethylsulfoxide (DMSO) [92] and mesityl oxide [93]. These are detected via UV absorption, not available on our instrument. Instead, two other techniques were devised: solid particle propagation and matrix-buffer boundary movement.

The solid particle approach was applied as follows: Approximately $200 \mathrm{mg}$ of nickel powder (Novamet 4SP-400) with a mean diameter of $10 \mu \mathrm{m}$ was mixed with $1 \mathrm{ml}$ of LPA by vortexing, centrifuged briefly to remove air bubbles, then pumped into the capillary. The movement of the particles could be observed in the instrument's onboard microscope, and images of the capillary were obtained with the video camera as described in section 3.2.3. The 
particle locations were recorded manually on the images and the resulting position vs. time and current data used to find the propagation coefficients using equation (4-5). The limitation of this technique was that the surface charge of the particles was unknown. It was, therefore, unclear whether the particles were moving because of EOF or electrophoresis. In order to check this, the position of the LPA/buffer boundary at the anode was recorded.

The moving LPA boundary method was based on the assumption that under normal well buffered conditions, the LPA itself did not gain significant charge so that any capillary wall-induced EOF would pump both the LPA and buffer towards the cathode. To observe this effect, the LPA at the anode was replaced with buffer at the start of the run, to make an LPA-liquid boundary. After the run, the capillary was cut up from the anode end and at each cutting, the end of the capillary was prodded with a piece of $50 \mu \mathrm{m}$ wire. Buffer by itself did not adhere to the wire, whereas the extremely sticky LPA would.

Three capillaries from three different bundles were tested with the nickel powder/matrix mixture and the particles were found to move between -1.2 and $-1.5 \mathrm{~m} / \mathrm{C}$ (the negative sign refers to the particles moving towards the cathode, the opposite of DNA). The rates were the same for all particles observed, and constant over runs up to 25 minutes long. The cut capillary method was subsequently used on one of the capillaries which showed the particles moving $-1.2 \mathrm{~m} / \mathrm{C}$. After a 160 minute run, the buffer/LPA interface was found $4 \mathrm{~cm}$ from the anode, again giving $-1.2 \mathrm{~m} / \mathrm{C}$. The fact that the particles move at the same rate as the LPA supports the hypotheses that the particles and LPA are uncharged.

The direct effect of the depletion region on the particles was then observed, and Figure 31 reveals the surprising result: crossing an anode boundary causes the particles to reverse direction. The particle 
tracked in Figure 31 initially moves towards the cathode at $-1.3 \mathrm{~m} / \mathrm{C}$. After the anode boundary passes through at $8 \mathrm{~m} / \mathrm{C}$, the same particle reverses and propagates towards the cathode at $4.6 \mathrm{~m} / \mathrm{C}$. The depletion depth in this region was estimated using equation (C-2), (Appendix $\mathrm{C}$ ) at $85 \mu \mathrm{S} / \mathrm{cm}$. The anode boundary moves faster than the particles, so they do not stack against the boundary. A similar effect is shown in Figure 32 where a dilute-buffer depletion region, estimated at $200 \mu \mathrm{S} / \mathrm{cm}$, caused particles to slow from $-1.2 \mathrm{~m} / \mathrm{C}$ to $-0.2 \mathrm{~m} / \mathrm{C}$.
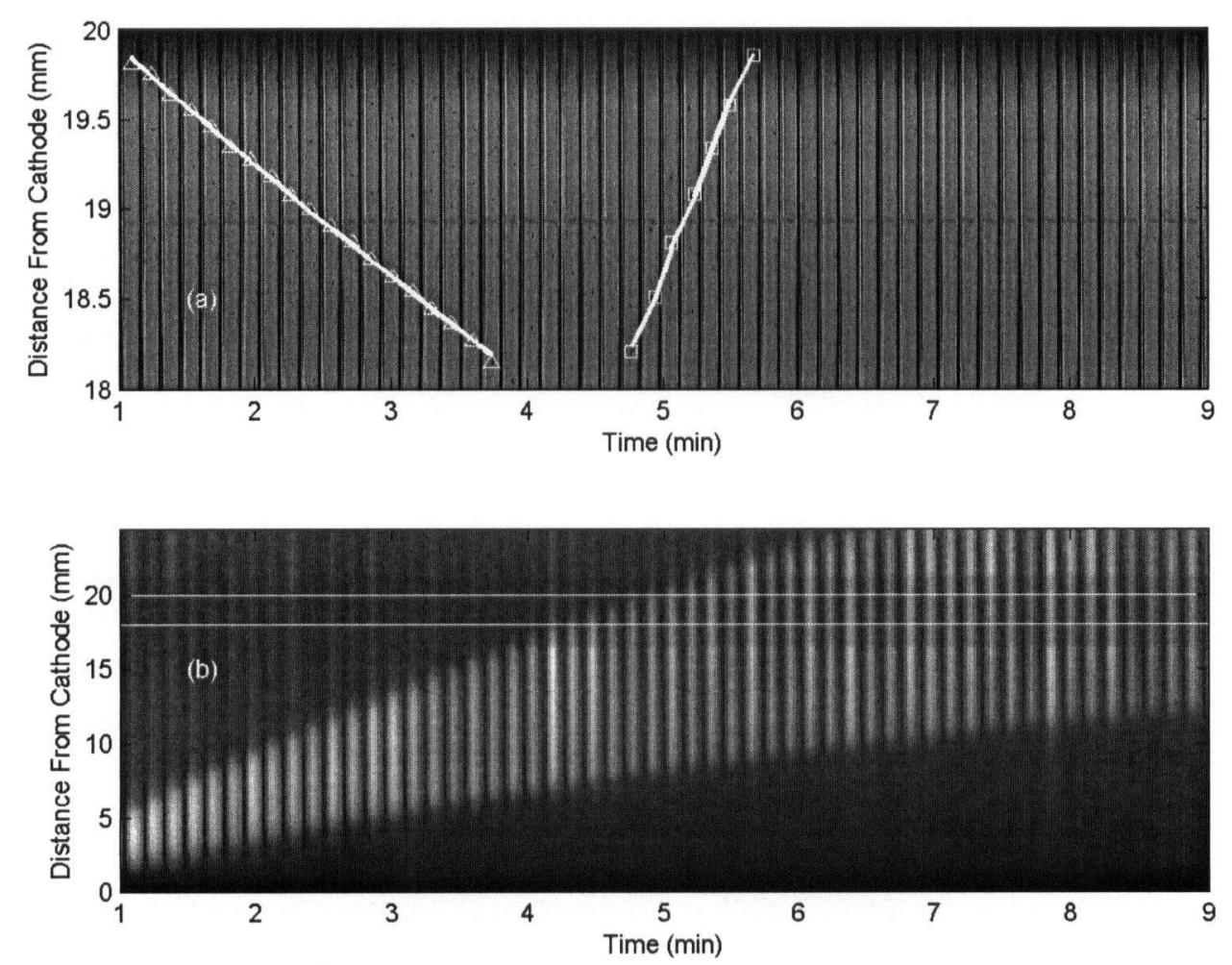

Figure 31 (a) Images of nickel particles in the capillary. The falling and rising white lines are tracks of one particle before and after being passed by the depletion boundary. The left particle track moves $-1.3 \mathrm{~m} / \mathrm{C}$ and the right one moves $4.6 \mathrm{~m} / \mathrm{C}$. The dark horizontal line in (a) is an artifact. (b) A thermal image of the same capillary. The horizontal while lines delineate the field of view of the visible images in (a). 

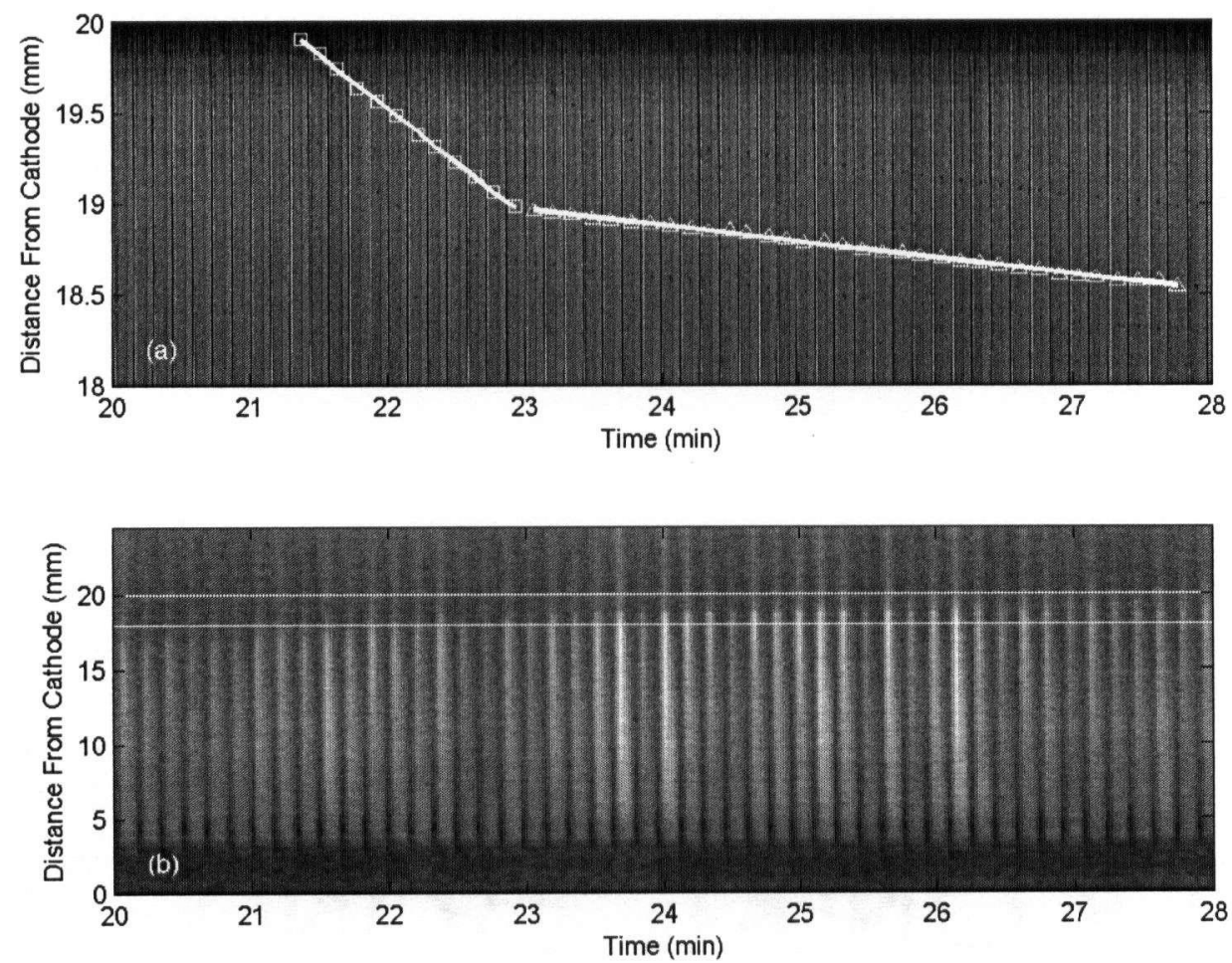

Figure 32 (a) Progress of nickel particles as a depletion boundary crosses their paths. Particles are visible as rows of black dots above and below the white lines, which track a third particle. Its propagation rate changes from $-1.2 \mathrm{~m} / \mathrm{C}$ to $-0.2 \mathrm{~m} / \mathrm{C}$. Note the lowest particle changes direction first and the upper ones follow in order. (b) A thermal image of the same capillary. The horizontal while lines delineate the field of view of the visible images in (a).

The behaviour of the particles as they cross into the depletion boundary is only consistent with a change in electrophoretic behaviour of the particles. Conservation of mass requires that it cannot be some local change in EOF direction. Figure 32 also shows that the downstream particles clearly change direction later than the particles closer to the cathode, the direction from which the depletion boundary is coming. This implies that the surface charge of the particles must 
change from neutral to negative. The nickel particles are likely to have a surface layer of nickel oxide. Nickel oxide, as other oxides, is known to have an isoelectric point where surface groups are charged. Parks [94] gives the isoelectric point for $\mathrm{NiO}$ as 10.3. It is possible to estimate if these groups are becoming charged, assuming the particles are moving at a terminal velocity, balanced between the electrophoretic force and viscous drag,

$$
F_{e}=F_{d}
$$

where the electrophoretic force is

$$
F_{e}=E q=f A_{p} \rho E,
$$

and where $E$ is the local field in the depletion region, estimated at $1.8 \times 10^{5} \mathrm{~V} / \mathrm{m}$ and $q$, the charge on the particle, is the maximum change density $\rho$ multiplied by the particle area $A_{p}$ and a surface charge density factor $f$. If the spacing of atoms at the surface is $3 \AA, \rho=1.7$ $\mathrm{Coul} / \mathrm{m}^{21}$, the viscous drag force is given by Stokes Law:

$$
F_{d}=6 \pi \eta r \vec{v}
$$

where $\eta$ is the viscosity, and $r$ is the particle radius of $10 \mu \mathrm{m}$. The particle velocity was found from Figure 31 (a) as velocity of the lefthand particle track added to the velocity of the right-hand particle track as the former represents the EOF. This gives $v=4 \times 10^{-5} \mathrm{~m} / \mathrm{s}$ or a shear rate of about $0.5 \mathrm{~s}^{-1}$. This corresponds to an LPA viscosity of about 50Pa s $[47,95]$. Solving for the fraction of sites $f$ which would have to be singly ionized to give the observed velocity, gives $f=0.4 \%$ which is certainly reasonably small. Without knowing more about details of the particles' surface chemistry we cannot accurately determine the rise in $\mathrm{pH}$. It suffices to say here that there is reasonable evidence that the $\mathrm{pH}$ does indeed rise in the depletion

\footnotetext{
${ }^{1}$ Published values for silanol groups on glass are $0.6-0.8 \mathrm{Coul} / \mathrm{m}^{2}[23]$
} 
region, and that the degree of increase is proportional to the depth of depletion.

\subsubsection{Boundary Movement by $\mathrm{OH}^{-}$Propagation}

In light of the evidence for rising $\mathrm{pH}$ in the depletion region, we examine one other possible model for the observed moving boundaries. Rising pH can occur naturally where the concentration of the acid component of a buffer solution is reduced while the basic component is maintained. In the case of a $50 \mathrm{mmol} / \mathrm{L}$ Tris/TAPS solution, the $\mathrm{pH}$ will rise from 8.3 to 10 as [TAPS] declines to zero. In an electrophoretic system, the boundary between high and normal $\mathrm{pH}$ regions can move because extra $\mathrm{OH}^{-}$ions impinging on the background region cause neutral TAPS to be ionized. This draws down the TAPS ion concentration and the boundary moves.

It is possible to derive a boundary velocity in this system. First, note that this velocity is different from that described in section 5.4.1 because in the present case of a buffer, ionic species are not conserved. Here, $\mathrm{OH}^{-}$ions from the depletion region travel into the undepleted region effectively adding extra base to the undepleted region. The excess $\mathrm{OH}^{-}$ions are neutralized by the ionization of TAPS $^{\circ}$ to TAPS: This is normal buffer behaviour, but because the system is under electrophoresis, the newly created TAPS ${ }^{-}$ions move. Thus the $\operatorname{TAPS}^{0}$ concentration is drawn down at a rate proportional to the flux of $\mathrm{OH}^{-}$ions. The boundary remains sharp: $\mathrm{OH}^{-}$diffusing forward are neutralized and TAPS $^{0}$ ions diffusing backward become TAPS - and move forward again. To calculate the expected boundary propagation rate, we make the assumption that the excess $\mathrm{OH}^{-}$ions in the depletion region can ionize the neutral TAPS on the undepleted side. 


$$
v_{O H}^{d}[O H]^{d} A=v_{b}[T A P S]^{b} A
$$

The subscript $d$ refers to the depletion region and $b$ to the nondepleted (background) region.

$$
\frac{\mu_{O H} I}{\sigma^{d} A}[O H]^{d} A=v_{b}[T A P S]^{b} A
$$

The boundary propagation rate in $\mathrm{m} / \mathrm{C}$ is therefore

$$
\frac{v_{b}}{I}=\frac{\mu_{O H}[O H]^{d}}{\sigma^{d}[T A P S]^{b} A}
$$

Equation (5-52) shows that deeper depletion will produce a faster moving boundary and the boundary moves faster if the background region is less conductive (Experimental evidence for the latter involving depleted matrices is shown in Appendix C.2). To actually use (5-52) requires knowledge of $\left[\mathrm{OH}^{-}\right]$and the depletion conductivity. Just calculating the ion concentrations from initial conditions does not take into account the increased concentration of Tris as it is transported into the depletion region and neutralized. Obtaining realistic values of these quantities requires actual simulation.

Simulations were done using the finite difference model with buffering, as described in 5.2, with initial capillary conditions [Tris] = [TAPS] $=50 \mathrm{mmol} / \mathrm{L}$ and the cathode boundary set to $[\mathrm{Tris}]=50$ $\mathrm{mmol} / \mathrm{L}$ and [TAPS] from .1 to $10 \mathrm{mmol} / \mathrm{L}$. Data from a three minute simulation with [TAPS] $=0.8 \mathrm{mmol} / \mathrm{L}$ is shown in Figure 33. The boundary is self-sharpening, and propagates at $12 \mathrm{~m} / \mathrm{C}$. The rise in neutral Tris from about $25 \mathrm{mmol} / \mathrm{L}$ in the background to $100 \mathrm{mmol} / \mathrm{L}$ in the depletion region can be seen, accompanied by a rise in $\mathrm{pH}$ from 8.3 to 10.2 . 


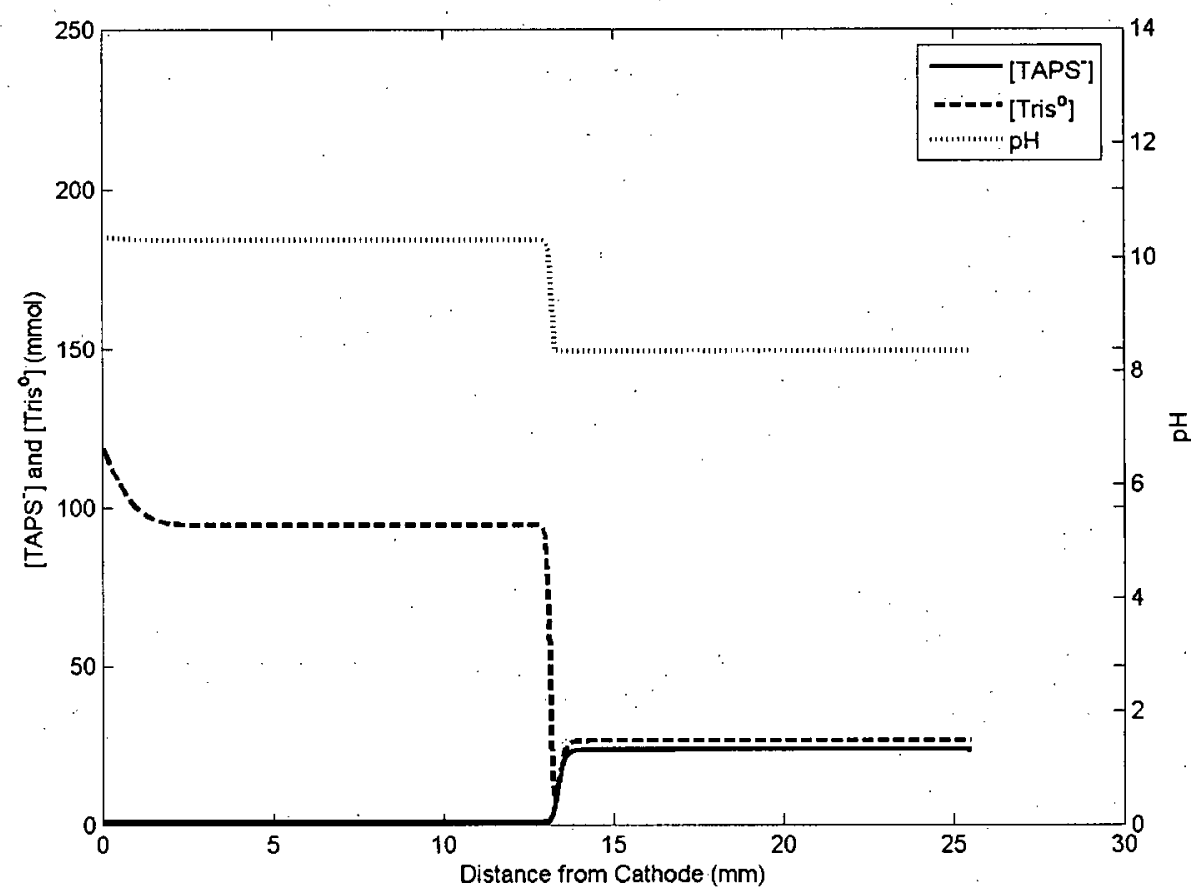

Figure 33 A five minute simulation with [Tris] $=50 \mathrm{mmol} / \mathrm{L}$ and [TAPS] $=0$ at cathode. As the boundary moves from left to right, the retreating TAPS ion is replaced by $\mathrm{OH}^{-}$ions, raising the $\mathrm{pH}$.

The comparison of calculated, simulated and experimental boundary propagation rates are shown in Figure 34. The calculated values were obtained (5-52) by using values for $\left[\mathrm{OH}^{-}\right]^{d}$ and $\sigma_{d}$ obtained by simulation, and those results closely match. An interesting effect of the simulations is that as [TAPS] drops below $0.4 \mathrm{mmol} / \mathrm{L}$, the conductivity actually rises. This is not observed in the experimental data. 


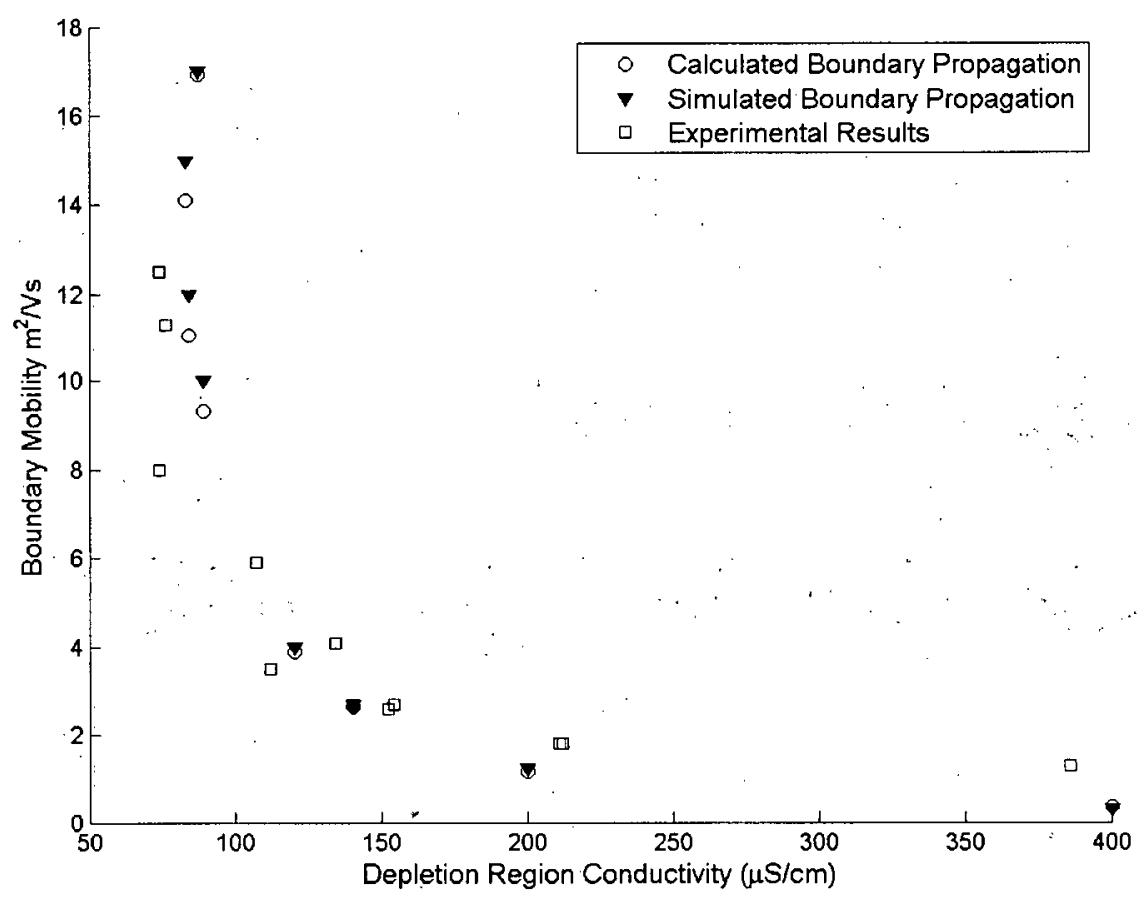

Figure 34 Simulations of the depleted cathode-side buffer for [TAPS] $=0.4$ to $8 \mathrm{mmol} / \mathrm{L}$ compared to experimental data. The capillary has $[$ Tris] $=[$ TAPS] $=50 \mathrm{mmol} / \mathrm{L}$ giving a conductivity of $1130 \mu \mathrm{S} / \mathrm{cm}$.

The obvious difficulty of this model is that it is not clear how such a large elevation in $\mathrm{pH}$ can arise. In the model, the cathode buffer is arbitrarily assigned to have a normal Tris concentration and depleted TAPS, which raises the $\mathrm{pH}$. In the case where DNA is present, the Tris and TAPS carrier ions should be depleted to the same degree unless the DNA is able to selectively block the TAPS ion and allow $\mathrm{OH}^{-}$through. Such behaviour is seen in macroions at membranes but is unlikely to operate with a small ion such as TAPS. Likewise depleted cathode buffers produce moving boundaries right away, not giving enough time for the inbound Tris to raise the $\mathrm{pH}$ in the buffer well itself. As we showed in the previous section however, there does appear to be a rise in the $\mathrm{pH}$ in the depletion region, so while the 
fixed charge model is more likely to be correct, the mismatched $\mathrm{pH}$ model cannot be completely disregarded until more is known about the $\mathrm{pH}$ in the depletion region. 


\section{Chapter 6 Conclusion}

This research has clarified for the first time the mechanism of current decline in DNA sequencing in capillary array electrophoresis. First, by analyzing current and fluorescence data from capillary array electropherograms, it was shown that current decline was the primary cause of reduced DNA read length rather than direct interference from contaminant fragments. Although the quality of the DNA peaks was preserved, current decline caused the velocity of the peaks to drop, meaning that fewer peaks arrived at the detector during the run.

It was also shown that while bubbles were found in sequencing capillaries, they were not the primary driving mechanism of current decline, and in fact, expanding bubbles are likely an effect rather than a cause of current decline. It was also shown that local capillary cooling, associated with opening the machine for buffer and sample exchanges, allowed small voids to form which permitted, in many cases, runaway bubble growth. Resuspending samples in agarose gel had previously been shown to prevent current decline. Further experiments here suggest that agarose reduces the overall quantity of injected DNA, without reducing the sample stacking efficiency, rather than preferentially reducing the loading of long fragments.

In order to study current decline in more detail, a single capillary apparatus was constructed. Using $48 \mathrm{~kb}$ double stranded $\lambda$ DNA as a model molecule and commercial linear polyacrylamide matrix with Tris/TAPS buffer, it was shown that there was a threshold for current decline of 10-15 ng of injected DNA. Capillary cutting and resistance measurement revealed that beyond the threshold, DNA induced an expanding region of ionic depletion. A novel technique of infrared imaging of the capillary was introduced enabling differences in conductivity to be observed, since the more depleted side was at 
elevated temperature. This enabled observation of the propagating concentration boundaries in real time. The DNA was found to propagate with the trailing edge of the depletion region while the leading edge propagated away. Combining these data with experiments with depleted buffers and no DNA revealed that the propagation rate of the leading boundary was proportional to the depth of the depletion region. This led to a hypothesis that the threshold for current decline occurred when enough DNA was injected to cause the leading boundary to propagate faster than the DNA itself. Subthreshold quantities of DNA would produce a smaller depletion in the downstream electrolyte so any leading boundary movement would be overtaken by the DNA itself.

Analytic and numerical models were introduced to demonstrate how semi-fixed charges - approximating DNA - cause a depletion region to form in the downstream background electrolyte. A numerical simulation showed that about $16 \mathrm{ng}$ of DNA was required to completely deplete the background electrolyte, in good agreement with experiment.

Models of anode and cathode boundary propagation were developed. The DNA, propagating with the cathode boundary, was found to change propagation regimes, from biased reptation to entropic trapping, with declining field. This explained the DNA and cathode boundary's propagation rate which was non-linear with current. The anode boundary was found to have a linear propagation rate with current, but the mechanism by which it moved was more difficult to determine. It was shown via simulation that the most likely propagation mode involved a mismatch in positive and negative carriers in the depletion region brought on by the presence of bound charges either on the capillary surface or in the matrix. This model, 
however, did not produce significant changes in $\mathrm{pH}$, which may in fact be present.

Indirect experimental evidence for a rise in $\mathrm{pH}$ was found through the observation of nickel particles in the matrix, which were found to change propagation direction upon entering the depletion region. This was believed to be due to a rise in $\mathrm{pH}$. For that reason, another model was proposed to explain the situation where the depletion region propagates because it has an elevated $\mathrm{pH}$, where $\mathrm{OH}^{-}$ ions became principle current carriers.

At the present time, current decline in DNA sequencing seems to be mostly under control through the injection of smaller sample quantities with better purification, and more fault tolerant, though less efficient, matrices. As the era of single molecule sequencing draws near, the share of capillary sequencing in the overall market will best be maintained by extending continuous average read lengths as far as possible. This will require more selective matrices, however, but these matrices must maintain a good tolerance to large fragments without exhibiting current decline.

It is clear that there is still much to learn about moving ionic boundaries. While the advent of narrow bore capillaries and commercial CZE instruments with UV detection has led to considerable research into conventional systems of sample and electrolyte peaks, questions remain, whether slow-moving boundaries can propagate merely in response to a change in the transference number of the principle carrier ions, and what are the relative contributions of $\mathrm{pH}$ changes, and bound charges in the matrix and capillary surface to these moving boundaries. Much of the research in this area dates back over seventy years; with modern CE tools and some of the techniques discussed here, these questions can and should be revisited more thoroughly. 


\section{Bibliography}

1. Ewing, B., et al., Base-calling of automated sequencer traces using phred. I. Accuracy assessment. Genome Rèsearch, 1998. 8(3): p. 175-185.

2. Collins, F.S., et al., $A$ vision for the future of genomics research. Nature, 2003. 422(6934): p. 835-847.

3. Hunkapiller, T., et al., Large-Scale And Automated DnaSequence Determination. Science, 1991. 254(5028): p. 59-67.

4. Holt, R.A., Personal Communication. 2005.

5. Chan, E.Y., Advances in sequencing technology. Mutation Research, 2005. 573(1-2): p. 13-40.

6. Shendure, J., et al., Accurate Multiplex Polony Sequencing of an Evolved Bacterial Genome. Science, 2005. 309: p. 17281732.

7. Margulies, M., Genome sequencing in microfabricated highdensity picolitre reactors. Nature, 2005. 437: p. 376-380.

8. Arratia, R., et al., Genomic Mapping By Anchoring Random Clones - A Mathematical-Analysis. Genomics, 1991. 11(4): p. 806-827.

9. Smailus, D., Personal Communication. 2002.

10. Zhou, H.H., et al., DNA sequencing up to 1300 bases in two hours by capillary electrophoresis with mixed replaceable linear polyacrylamide solutions. Analytical Chemistry, 2000. 72(5): p. 1045-1052.

11. Jovanovich, S.B., Personal Communication. 2001. 
12. McArdle, B.F., et al. Analysis of Factors Influencing Capillary Sequencing with the MegaBACE 1000. in Advances in Genome Biology and Technology. 2000. Marco Island FL USA.

13. Salas-Solano, O., et al., $A$ sample purification method for rugged and high-performance DNA sequencing by capillary electrophoresis using replaceable polymer solutions. $B$. Quantitative determination of the role of sample matrix components on sequencing analysis. Analytical Chemistry, 1998. 70(8): p. 1528-1535.

14. Ruiz-Martinez, M.C., et al., A sample purification method for rugged and high-performance DNA sequencing by capillary electrophoresis using replaceable polymer solutions. $A$. Development of the cleanup protocol. Analytical Chemistry, 1998. 70(8): p. 1516-1527.

15. Karger, B.L., Some personal words on the career of Stellan Hjerten - Commemoration. Journal of Chromatography A, 2003. 1004(1-2): p. 7-8.

16. Jorgenson, J.W. and K.D. Lukacs, Zone Electrophoresis In Open-Tubular Glass-Capillaries. Analytical Chemistry, 1981. 53(8): p. 1298-1302.

17. Hu, S. and N.J. Dovichi, Capillary electrophoresis for the analysis of biopolymers. Analytical Chemistry, 2002. 74(12): p. 2833-2850.

18. Sartori, A., V. Barbier, and J.L. Viovy, Sieving mechanisms in polymeric matrices. Electrophoresis, 2003. 24(3): p. 421-440.

19. Slater, G.W., P. Mayer, and G. Drouin, Migration of DNA through gels, in Methods in Enzymology: High Resolution Separation and Analysis of Biological Macromolecules, Pt A. 1996. p. 272-295. 
20. Quesada, M.A., Replaceable polymers in DNA sequencing by capillary electrophoresis. Current Opinion in Biotechnology, 1997. 8(1): p. 82-93.

21. Guttman, A. and N. Cooke, Effect of Temperature on the Separation of DNA Restriction Fragments in Capillary GelElectrophoresis. Journal of Chromatography, 1991. 559(1-2): $p$. 285-294.

22. Luckey, J.A., T.B. Norris, and L.M. Smith, Analysis of Resolution in DNA Sequencing by Capillary Gel-Electrophoresis. Journal of Physical Chemistry, 1993. 97(12): p. 3067-3075.

23. Hirokawa, T., et al., Change of migration time and separation window accompanied by field-enhanced sample stacking in capillary zone electrophoresis. Electrophoresis, 2001. 22(16): p. 3444-3448.

24. Dolnik, V., Wall coating for capillary electrophoresis on microchips. Electrophoresis, 2004. 25(21-22): p. 3589-3601.

25. Barbier, V. and J.L. Viovy, Advanced polymers for DNA separation. Current Opinion in Biotechnology, 2003. 14(1): p. 51-57.

26. Heller, C., Principles of DNA separation with capillary electrophoresis. Electrophoresis, 2001. 22(4): p. 629-643.

27. Stellwagen, E. and N.C. Stellwagen, The free solution mobility of DNA in Tris-acetate-EDTA buffers of different concentrations, with and without added $\mathrm{NaCl}$. Electrophoresis, 2002. 23(12): p. 1935-1941.

28. Jain, M., Personal Communication. 2000.

29. Xiong, Y., S.R. Park, and H. Swerdlow, Base stacking: $p H$ mediated on-column sample concentration for capillary DNA sequencing. Analytical Chemistry, 1998. 70(17): p. 3605-3611. 
30. Sanger, F., S. Nicklen, and A.R. Coulson, DNA Sequencing with Chain-Terminating Inhibitors. Proceedings of the National Academy of Sciences of the United States of America, 1977. 74(12): p. 5463-5467.

31. Maxam, A.M. and W. Gilbert, New Method for Sequencing DNA. Proceedings of the National Academy of Sciences of the United States of America, 1977. 74(2): p. 560-564.

32. Smith, L.M., et al., Fluorescence Detection in Automated DNASequence Analysis. Nature, 1986. 321(6071): p. 674-679.

33. Swerdlow, H. and R. Gesteland, Capillary Gel-Electrophoresis for Rapid, High-Resolution DNA Sequencing. Nucleic Acids Research, 1990. 18(6): p. 1415-1419.

34. Cohen, A.S., D.R. Najarian, and B.L. Karger, Separation and Analysis of DNA-Sequence Reaction-Products by Capillary GelElectrophoresis. Journal of Chromatography, 1990. 516(1): $p$. 49-60.

35. Drossman, H., et al., High-Speed Separations of DNA Sequnencing Reactions by Capillary Electrophoresis. Analytical Chemistry, 1990. 62: p. 900-903.

36. Guttman, A., et al., Analytical and Micropreparative Ultrahigh Resolution of Oligonucleotides by Polyacrylamide-Gel HighPerformance Capillary Electrophoresis. Analytical Chemistry, 1990. 62(2): p. 137-141.

37. Ju, J.Y., et al., Design and Synthesis of Fluorescence EnergyTransfer Dye-Labeled Primers and Their Application for DNASequencing and Analysis. Analytical Biochemistry, 1995. 231(1): p. 131-140.

38. Mathies, R.A. and X.C. Huang, Capillary Array Electrophoresis an Approach to High-Speed, High-Throughput DNA Sequencing. Nature, 1992. 359(6391): p. 167-169. 
39. Swerdlow, H., et al., Capillary Gel-Electrophoresis for DNA Sequencing - Laser-Induced Fluorescence Detection with the Sheath Flow Cuvette. Journal of Chromatography, 1990. 516(1): p. 61-67.

40. Luckey, J.A., et al., High-Speed DNA Sequencing by Capillary Electrophoresis. Nucleic Acids Research, 1990. 18(15): p. $4417-$ 4421.

41. Yin, H.F., J.A. Lux, and G. Schomburg, Production of Polyacrylamide-Gel Filled Capillaries for Capillary GelElectrophoresis (Cge) - Influence of Capillary Surface Pretreatment on Performance and Stability. Hrc-Journal of High Resolution Chromatography, 1990. 13(9): p. 624-627.

42. Swerdlow, H., et al., Stability of Capillary Gels for Automated Sequencing of DNA. Electrophoresis, 1992. 13(8): p. 475-483.

43. Figeys, D., A. Renborg, and N.J. Dovichi, Spatial and Temporal Depletion of lons from Noncrosslinked Denaturing Polyacrylamide in Capillary Electrophoresis. Electrophoresis, 1994. 15(12): p. 1512-1517.

44. Figeys, D. and N.J. Dovichi, Change in conductivity in noncross-linked polyacrylamide capillary electrophoresis - Effects of aging polyacrylamide and buffer composition. Journal of Chromatography A, 1996. 744(1-2): p. 333-339.

45. Carrilho, E., et al., Rapid DNA sequencing of more than 1000 bases per run by capillary electrophoresis using replaceable linear polyacrylamide solutions. Analytical Chemistry, 1996. 68(19): p. 3305-3313.

46. Ruiz-Martinez, M.C., et al., DNA-Sequencing by Capillary Electrophoresis with Replaceable Linear Polyacrylamide and Laser-Induced Fluorescence Detection. Analytical Chemistry, 1993. 65(20): p. 2851-2858. 
47. Goetzinger, W., et al., Characterization of high molecular mass linear polyacrylamide powder prepared by emulsion polymerization as a replaceable polymer matrix for DNA sequencing by capillary electrophoresis. Electrophoresis, 1998. 19(2): p. 242-248.

48. Dolnik, V., et al., Wall coating for DNA sequencing and fragment analysis by capillary electrophoresis. Journal of Microcolumn Separations, 1998. 10(2): p. 175-184.

49. Karger, A.E., Separation of DNA sequencing fragments using an automated capillary electrophoresis instrument. Electrophoresis, 1996. 17(1): p. 144-151.

50. Madabhushi, R., Separation of 4-color DNA sequencing extension products in non-covalently coated capillaries, using low viscosity polymer solutions. Electrophoresis, 1998. 19: $\mathrm{p}$. 224-230.

51. Doherty, E.A.S., et al., Critical factors for high-performance physically adsorbed (dynamic) polymeric wall coatings for capillary electrophoresis of DNA. Electrophoresis, 2002. 23(16): p. 2766-2776.

52. Good, N.E., et al., Hydrogen lon Buffers for Biological Research. Biochemistry, 1966. 5(2): p. 467-477.

53. Siruno, A., et al. Sensitivity of the Applied Biosystems 3730 and 3730xI DNA Analysers. in Advances in Genome Biology and Technology conference (AGBT). 2003. Marco Island, FL, USA.

54. Waterston, R.H., E.S. Lander, and J.E. Sulston, On the sequencing of the human genome. Proceedings of the National Academy of Sciences of the United States of America, 2002. 99(6): p. 3712-3716.

55. Waterston, R.H., E.S. Lander, and J.E. Sulston, More on the sequencing of the human genome. Proceedings of the National 
Academy of Sciences of the United States of America, 2003. 100(6): p. 3022-3024.

56. Collins, F. and D. Galas, A New 5-Year Plan For The UnitedStates Human Genome Project. Science, 1993. 262(5130): p. 43-46.

57. Johnson, B.F., Entrapment of nucleic acid sequencing template in sample mixtures by entangled polymer networks. 2000, US Patent 6051636, Applied Biosystems: USA.

58. Vatcher, G., et al., Resuspension of DNA sequencing reaction products in agarose increases sequence quality on an automated sequencer. Biotechniques, 2002. 33(3): p. 532-538.

59. Huang, X.H.C., M.A. Quesada, and R.A. Mathies, Capillary Array Electrophoresis Using Laser-Excited Confocal Fluorescence Detection. Analytical Chemistry, 1992. 64(8): p. 967-972.

60. Burgi, D.S., Personal Communication. 2000.

61. Ewing, B. and P. Green, Base-calling of automated sequencer traces using phred. II. Error probabilities. Genome Research, 1998. 8(3): p. 186-194.

62. Bilenko, O., et al., Formation of a resistive region at the anode end in DNA capillary electrophoresis. Electrophoresis, 2003. 24(7-8): p. 1176-1183.

63. Heller, C., Influence of electric field strength and capillary dimensions on the separation of DNA. Electrophoresis, 2000. 21(3): p. 593-602.

64. Li, D.M., S.L. Fu, and C.A. Lucy, Prediction of electrophoretic mobilities. 3. Effect of ionic strength in capillary zone electrophoresis. Analytical Chemistry, 1999. 71(3): p. 687-699. 
65. Desruisseaux, C., G.W. Slater, and G. Drouin, The gel edge electric field gradients in denaturing polyacrylamide gel electrophoresis. Electrophoresis, 1998. 19(5): p. 627-634.

66. Isambert, H., et al., Electrohydrodynamic patterns in macroion dispersions under a strong electric field. Physical Review E, 1997. 56(5): p. 5688-5704.

67. Mikkers, F.E.P., Concentration distributions in capillary zone electrophoresis: CZE in a spreadsheet. Analytical Chemistry, 1999. 71(2): p. 522-533.

68. Gas, B., et al., PeakMaster - A freeware simulator of capillary zone electrophoresis. LC GC Europe, 2005. 18(5): p. 282-+.

69. Hopkins, D.L. and V.L. McGuffin, Three-dimensional molecular simulation of electrophoretic separations. Analytical Chemistry, 1998. 70(6): p. 1066-1075.

70. McKinley, J.D. and C. Savvidou, Numerical Modelling of the Electrophoretic Remediation of Heavy Metal Contaminated Soil. CUED/D-Soils, 1997. TR292.

71. Ermakov, S.V., S.C. Jacobson, and J.M. Ramsey, Computer simulations of electrokinetic transport in microfabricated channel structures. Analytical Chemistry, 1998. 70(21): p. 4494-4504.

72. Beckers, J.L., M. Urbanek, and P. Bocek, System zones in capillary zone electrophoresis: Moving boundaries caused by freely migrating hydroxide ions. Electrophoresis, 2005. 26(10): p. 1869-1873.

73. Ng, B. and P.H. Barry, The measurement of ionic conductivities and mobilities of certain less common organic ions needed for junction potential corrections in electrophysiology. Journal of Neuroscience Methods, 1995. 56: p. 37-41.

74. Ikuta, N. and T. Hirokawa, Numerical simulation for capillary electrophoresis I. Development of a simulation program with 
high numerical stability. Journal of Chromatography A, 1998. 802(1): p. 49-57.

75. Marziali, A., et al., Novel electrophoresis mechanism based on synchronous alternating drag perturbation. Electrophoresis, 2005. 26(1): p. 82-90.

76. Viovy, J.L., Electrophoresis of DNA and other polyelectrolytes: Physical mechanisms. Reviews of Modern Physics, 2000. 72(3):

p. $813-872$

77. Duke, T., et al., Sequencing in nanofabricated arrays: $A$ feasibility study. Electrophoresis, 1997. 18(1): p. 17-22.

78. Zhang, Y., H.J. Zhou, and Z.C. Ou-Yang, Stretching singlestranded DNA: Interplay of electrostatic, base-pairing, and basepair stacking interactions. Biophysical Journal, 2001. 81(2): p. 1133-1143.

79. Kohlrausch, F., Ueber Concentrations-Verschiebungen durch Electrolyse im Inneren von Losungen und Losungsgemischen. Annalen der Physik, 1897. 62: p. 209-239.

80. Macinnes, D.A. and L.G. Longsworth, Transferrence Numbers by the Method of Moving Boundaries. Chemical Reviews, 1932. 11(2): p. 171-230.

81. Spencer, M., Anomalous Conductivity Zones in Electrophoresis.1. Basic Theory for 2-Ion Systems. Electrophoresis, 1983. 4(1): p. 36-41.

82. Spencer, M., Anomalous Conductivity Zones in Electrophoresis.2. Theory for 3-Ion Systems and for Changes in Ph. Electrophoresis, 1983. 4(1): p. 41-45.

83. Spencer, M. and J.M. Kirk, Anomalous Conductivity Zones in Electrophoresis.3. Experimental Tests of the Theory. Electrophoresis, 1983. 4(1): p. 46-52. 
84. Smith, E.R., Bureau of Standards Journal of Research, 1931. 6: p. 917-26.

85. Chien, R.L., Sample stacking revisited: A personal perspective. Electrophoresis, 2003. 24(3): p. 486-497.

86. Beckers, J.L., P. Gebauer, and P. Bocek, System zones in capillary zone electrophoresis. Electrophoresis, 2001. 22(17): p. 3648-3658.

87. Cao, C.X., Comparisons of the mobilities of salt ions obtained by the moving boundary method and two empirical equations in capillary electrophoresis. Journal of Chromatography A, 1997. 771(1-2): p. 374-378.

88. Longsworth, L., Moving Boundary Studies on Salt Mixtures. Journal of the American Chemical Society, 1945. 67: p. 11091119.

89. Figeys, D. and N.J. Dovichi, Effect of the Age of Non-CrossLinked Polyacrylamide on the Separation of DNA-Sequencing Samples. Journal of Chromatography A, 1995. 717(1-2): p. 105111.

90. Tanaka, T., et al., Phase Transitions in ionic Gels. Physical Review Letters, 1980. 45(20): p. 1636-1639.

91. Chiari, M., et al., Towards New Formulations For Polyacrylamide Matrices - N-Acryloylaminoethoxyethanol, A Novel Monomer Combining High Hydrophilicity With Extreme Hydrolytic Stability. Electrophoresis, 1994. 15(2): p. 177-186.

92. Cao, C.X., et al., Quantitative predictions to conditions of zwitterionic stacking by transient moving chemical reaction boundary created with weak electrolyte buffers in capillary electrophoresis. 2005. 77(4): p. 955-963.

93. Barron, A.E., H.W. Blanch, and D.S. Soane, A Transient Entanglement Coupling Mechanism For Dna Separation By 
Capillary Electrophoresis In Ultradilute Polymer-Solutions. 1994. 15(5): p. 597-615.

94. Parks, G.A., Isoelectric Points Of Solid Oxides Solid Hydroxides And Aqueous Hydroxo Complex Systems. 1965. 65(2): p. 177\&.

95. Albargheuthi, M.N. and A.E. Barron, Polymeric matrices for DNA sequencing by capillary electrophoresis. 2000. 21(18): $\mathrm{p}$. 4096-4111.

96. Schein, J., et al., Bacterial Artificial Chromosomes: Library Construction, Physical Mapping and Sequencing, in Methods in Molecular Biology, S. Zhao and M. Stodolsky, Editors, Humana Press: Totowa NJ. p. 143-156.

97. Ouellette, F., Personal Communication. 2005.

98. Birren, B., Personal Communication. 2005.

99. Burgi, D., K. Solomon, and R.L. Chien, Methods for Calculating the internal temperature of capillary collumns during capillary electrophoresis. Journal of Liquid Chromatography, 1991. 14(5): p. 847-867. 


\section{Appendix A Properties of DNA and DNA Sequence Methods}

\section{A.1 Properties of DNA}

In order to understand the constraints on DNA sequencing machines and protocols, the properties of DNA needs to be understood in detail. DNA has numerous properties that can be ingeniously employed to facilitate selective cutting, copying and amplification. It is the most stable of the major biomolecules to work with and, in contrast to proteins and metabolites, DNA fragments of different sequences have the same key properties such as shape and charge density. As a result, genomics, the large scale study of DNA, has advanced far and fast in comparison to "proteomics" or "metabolomics". Following are general descriptions of the useful properties of DNA, clone library construction and cycle sequencing and a detailed description of the methods of sample preparation used in this work.

Double stranded DNA is comprised of two polymeric helices made from four deoxynucleotides: Adenine, Guanine, Thymine and Cytosine ( $A, G, T$ and $C$ ) attached to a sugar phosphate backbone. $A$ and $T$ and $G$ and $C$ are each structurally complementary and noncovalently bond to each other, so in the double helix, $A$ is always opposite $T$ and $G$ opposite $C$. This creates excellent binding specificity as the probability of a sequence of $\mathrm{N}$ random nucleotides being complementary to another is $\mathrm{N}^{-4}$. Long double strands of DNA can be melted at $95^{\circ} \mathrm{C}$ into single strands, and annealed again at $50^{\circ} \mathrm{C}$. DNA can be copied by polymerase enzymes which extend a complementary copy along one of the single strands. The deoxyribose backbone of 
the DNA has 5 carbon atoms with hydroxyl groups on the $3^{\prime}$ and $5^{\prime}$ carbons and extension always occurs from the 3 ' end of the extending fragment towards the 5 ' end of the complementary fragment. There also exist restriction enzymes, which cut DNA at sites with DNA sequence unique to each enzyme.

Genomes of different organisms vary in size, from a few million DNA base pairs for bacteria to a few gigabases for mammals to ten gigabases for certain plants. The DNA is packed into chromosomes which can be extracted from cells and purified for analysis. Near random sequences of DNA are easiest to sequence, but the high binding strength of the $G$ and $C$ nucleotides make so-called " $G C$ rich regions" difficult to sequence. Regions with many short repeated sequences are also difficult as single stranded copies can loop back and undergo complementary binding with themselves. Special reaction conditions can be used to overcome these difficult regions.

\section{A.2 Library Construction}

There are directed and random strategies for dividing a genome up for sequencing, but all ultimately require the production of fragments a few kilobases in length. These must then be separated and individually amplified to produce enough starting material to perform the cycle sequencing reaction to produce the 20 to $1000 \mathrm{bp}$ fragments which will actually reveal the sequence. Overlapping sequence data from these individual fragments are then reassembled into the genome in silico. The lowest cost and most bias-free method of genome sequencing involves preparing libraries of randomly sheared fragments which are stored in $E$. coli cells which can be amplified as needed. These are called clone libraries.

To make clone libraries, randomly sheared fragments are ligated into a break in a circular piece of DNA called a vector (either a 
plasmid or a phage), capable of reproducing in bacterial cells. The unknown fragment plus vector, ligated back into a circle, is called the template, because copies will be made from it for sequencing. The template is then incorporated into $E$. coli cells by creating holes in the cell walls, either chemically, or through an electrical process called electroporation, which allows the template to diffuse into the cells - a process called transformation for prokaryotic cells. The concentration of template and $E$. coli are adjusted so that essentially all the successful transformations involve one vector only entering a cell. The cells are then "plated out" on agar gel based growth media. Surviving cells capable of reproduction can then establish individual colonies, where each daughter cell has a common ancestor, and contains a common template. The original vector into which the inserts were ligated contains one gene with resistance to antibiotics and another, (usually Lac-Z) which makes a blue pigment. The colony growth media contains ampicillin, so only the cells which incorporated a vector with the antibiotic gene survive. The inserts are actually ligated right into the middle of the Lac- $Z$ gene so the cells that incorporated a vector containing no insert survive, but appear blue, while the vectorplus-insert colonies have a non-functioning Lac- $Z$ gene and are white. The white colonies, each containing about $10^{6}$ cells, can then be picked from the growth media using a robot. Those colonies are grown further in 384 well incubation plates so enough of each colony exists that the insert can be extracted. These arrays of cell colonies in 384 well plates, each well containing one vector and DNA fragment of interest are called clone libraries. The advantage of maintaining the library as template in E. coli cells is that cells from each colony can be stored, re-plated, grown and sequenced as needed.

The best common approach to large scale sequencing today is to actually construct the libraries in two steps. A clone library of 
random fragments of order $100 \mathrm{~kb}$ are ligated into bacterial artificial chromosome (BAC) vectors and grown in a similar way to the shorter plasmid vectors. These BACs, whose locations with respect to each other are found through a process called restriction fragment mapping, are then sheared into random fragments, to make sequencing clone libraries.

\section{A.3 DNA Purification}

The next step in preparing sequence fragments is taking some of the clone libraries and extracting the template. A typical low-cost protocol, and one used in the present experiments, is the alkaline-lysis approach [96]. First, some cells are transferred from the clone library to new plates which are incubated again produce more DNA. These cells are then centrifuged into a pellet, the supernatant discarded and the cells resuspended in buffer (usually Tris-HCl) and EDTA (ethylenediaminetetraacetic acid). EDTA chelates divalent metals ions, which inhibits DNAses (enzymes that cut DNA).

The cells are then lysed with sodium hydroxide $(\mathrm{NaOH})$ and sodium dodecyl(lauryl)sulfate (SDS, a common detergent). The SDS makes holes in the cell membranes and the $\mathrm{NaOH}$ denatures the cellular DNA, separating it into single stranded fragments (sSDNA). The template DNA is circular however, so is topologically constrained. Potassium acetate (KAC) is then added, which does three things: The circular DNA is allowed to renature but sheared cellular DNA remains denatured. The ssDNA is precipitated, since large ssDNA molecules are insoluble in high salt. Adding KAc to the SDS also forms KDS, which is insoluble. This allows for the easy removal of the SDS from the plasmid DNA. The sample is again centrifuged to remove cell debris, KDS and cellular ssDNA. The template, and potentially some 
genomic DNA, is in the supernatant, while all other unwanted material is in the pellet.

The supernatant containing the DNA of interest must then be cleaned of excess salts. Ethanol or isopropanol and a salt - usually ammonium acetate - are added to the solution causing the DNA to precipitate out of solution while other ions remain. Centrifugation collects the DNA in a pellet at the bottom of the well at which point it can be resuspended in buffer, ready for cycle sequencing.

\section{A.4 Cycle Sequencing}

The method of DNA sequencing by electrophoresis was developed by Sanger [30] and independently by Gilbert and Maxam [31], in 1977. Although the original protocol is still in use in low throughput settings, it involves radio-labelled nucleotides and a separate separation must be done for each of the four bases. A significant advance occurred in 1986 when Hood et al. [32] reported laser-induced fluorescence (LIF) detection methods for DNA. With four different flourophores, separation was now possible in one lane rather than four, with no radiation handling issues. This sequencing protocol is in wide use, and is carried out in the following way:

Template (the vector plus insert described above) DNA is mixed with deoxyneucleotides (dNTPs: , A, C, G, and T), dideoxyneucleotides (ddNTPs), DNA polymerase (enzymes which add complementary dNTPs and ddNTPs to a strand of DNA) and a $\sim 20 \mathrm{bp}$ primer. The primer is complementary to a region on the template strand immediately adjacent to the insert, so any insert can be copied regardless of sequence. The key to this method is the ddNTPs, which differ from dNTPs by the lack of a 3' hydroxyl group. This prevents another nucleotide being added after a ddNTP is incorporated. The 
ddNTPs are also fluorescently labeled, with a different colour for each of the four nucleotides.

The reaction occurs at three temperatures. Above $90^{\circ} \mathrm{C}$, the DNA denatures, becoming single stranded. The temperature is then lowered to $50^{\circ} \mathrm{C}$, and primers bind to the template. The temperature is then raised to $60^{\circ} \mathrm{C}$ and the polymerases begin extending the primer with the available dNTPs, making a complementary copy of the insert. Crucially, the dNTPs and ddNTPs are mixed in a proportion of about 100:1. As a result, some fragments of each size are produced, each sharing a common starting point but terminated at a different nucleotide. As each terminating nucleotide has a dye molecule specific to its type, $A, C, G$ or $T$, when the fragments are separated by length, the terminating nucleotides can be identified by colour. Cycle sequencing reactions typically use thirty thermal cycles to generate enough DNA for sequencing. After thermal cycling, samples are again cleaned up with ethanol precipitation, and then dried down in their reaction plates, ready for sequencing. Just before loading the plates onto the sequencers, the samples are resuspended in $\mathrm{dH}_{2} \mathrm{O}$.

The full protocol normally involves aliquoting the ethanol purified template into two new plates before cycle sequencing and then performing the cycle sequencing reactions using one primer specific to each end of the template in each plate. The two sequences that result are complementary to each other, and may or may not overlap, depending on the length of the insert.

Sequencing data is assembled into actual DNA sequence by comparing different pieces of sequence to find where they overlap. Overlapping pieces can be assembled into contigs of continuous overlapping data. For a purely random genome, the assembly error probability is 


$$
P_{e}=e^{\frac{-N L}{G}}
$$

where $N$ is the number of reads, $L$ is the average read length, and $G$ is the total length of DNA to be reassembled [8]. Clearly longer read lengths $L$ and a greater number of reads $N$ are desirable. In fact for high quality genome sequencing, at least five times overlap is required. Sequencing via BAC maps, where the location in the genome of each $100-200 \mathrm{~kb} B A C$ is known, reduces the value of $\mathrm{G}$ from order $10^{9}$ to $10^{5}$. This is in fact a principle source of contention in the Celera/HGP debate as it has been argued that Celera Genomic's "whole genome shotgun" approach would never have worked had they not used the Human Genome Project's framework to enhance their accuracy. Interestingly, the error probability for real genomes, which are up to $10 \%$ repeats, has not be been formally studied. It is postulated that as many key repeat intervals in genomic DNA are on the order of $1 \mathrm{~kb}$, so read lengths of $1.5 \mathrm{~kb}$ would provide a significant advantage, but this has never been quantified [97],[98].

\section{A.5 Genome Sciences Center Sample Preparation}

The samples prepared for experiments performed on the MegaBACE were prepared in the following manner. This protocol is reproduced from Vatcher et al. [58] which details experiments in resuspending samples in dilute agarose to prevent current decline.

Further results stemming from this work are reported in section 4.1 and Appendix B.1.

Bacteria containing a single clone from the Mammalian Gene Collection (MGC-10790) were inoculated into each well of a 96-well culture block (Beckman Coulter) in $1.2 \mathrm{~mL}$ of $2 \mathrm{xYT}$ media (Becton Dickinson) supplemented with Chloramphenicol (Sigma) at 12.5 $\mu \mathrm{g} / \mathrm{mL}$. The block was sealed with a sheet of AirPore ${ }^{T M}$ tape (Qiagen) and incubated for 16 hours at $37^{\circ} \mathrm{C}$ with agitation at $290 \mathrm{rpm}$ in a New 
Brunswick Scientific shaking-incubator fitted with custom holders. Following growth, cell pellets were collected by centrifugation for 20 minutes at $1400 \mathrm{~g}$ and the media decanted. The plasmid DNA was then isolated using the modified alkaline lysis procedure described above.

Cycle sequencing was performed using DYEnamic energy transfer (ET) dye brew mix (Amersham Biosciences). To increase the speed and efficiency of fluid transfers and decrease well-to-well variability all fluid transfer steps were performed with Hydra 96-channel microdispensers (Robbins Scientific). All solutions and reactions were prepared with deionized $18 \mathrm{M} \Omega \mathrm{cm}$ water. $6 \mu \mathrm{L}$ of the homogenized template DNA ( $164 \mu \mathrm{g} / \mathrm{mL}$ ) was sequenced in each well of 2896 -well PCR plates. Each $20 \mu \mathrm{L}$ reaction contained $8 \mu \mathrm{L}$ of DYEnamic ET brew mix, 5 pmol of $-21 \mathrm{M} 13$ forward primer, and $6 \mu \mathrm{L}$ of DNA, the remaining volume was $\mathrm{dH}_{2} \mathrm{O}$. Cycle sequencing was performed in a PTC-225 DNA engine tetrad (MJ Research) with a ramp speed of $3^{\circ} \mathrm{C} / \mathrm{sec}$ using 30 cycles of $95^{\circ} \mathrm{C}$ for $20 \mathrm{~s}, 48^{\circ} \mathrm{C}$ for $15 \mathrm{~s}, 60^{\circ} \mathrm{C}$ for 1 min, followed by incubation at $4^{\circ} \mathrm{C}$.

For the experiments in agarose resuspension, the sequencing reactions from all plates were collected by centrifugation after thermal cycling, pooled and re-aliquoted into 28 fresh 96-well Robbins plates. To concentrate the sequenced products and remove extra salts and dye-terminators, the reactions were ethanol precipitated. $60 \mu \mathrm{L}$ of $95 \%$ ethanol and $2 \mu \mathrm{L}$ of $7.5 \mathrm{~mol} / \mathrm{L}$ ammonium acetate $(\mathrm{pH} 7.5)$ were added to each well and, after mixing by repeated pipetting with a Robbins Hydra, DNA precipitates were collected by centrifuging the cycle plate for $30 \mathrm{~min}$ at $2750 \mathrm{~g}$ at $4{ }^{\circ} \mathrm{C}$. The ethanol/salt mixture was decanted immediately following centrifugation by inverting and vigorously shaking the plates to remove the liquid from the wells. Following a wash with $150 \mu \mathrm{L}$ of $70 \%$ ethanol the plates were spun inverted at 700 
g over paper towelling for $1 \mathrm{~min}$ to remove any residual ethanol. The reaction pellets were dried in a SpeedVac with the rotor removed, on high heat for 2 minutes. The plates were sealed with foil tape and the precipitated sequencing reactions were stored at $-20^{\circ} \mathrm{C}$ in plastic bags.

For the experiments in bubble growth and propagation reported in section 4.2 and Appendix C, 96 well plates with ethanol purified template dried down and stored at $-20^{\circ} \mathrm{C}$ were received at the Marziali lab, and cycle sequencing and ethanol precipitation were performed as described above. The only difference was that reagents were added manually using a single channel Gilson Distriman manual pipettor and an eight channel electric pipettor instead of a Robbins Hydra. The samples were also not pooled and realiquoted after cycle sequencig 


\section{Appendix B The Effect of \\ Sample Resuspension in}

\section{Agarose}

\section{B.1 Effects of Injection from Agarose}

An initial motivation for research into current decline was to find methods to mitigate current decline with minimal added cost in sample preparation steps, reagents or hardware. Reducing the concentration of long fragments through restriction digests or filtration had been reported $[14,42,57]$ but none had been widely adopted by genome centers, presumably for cost reasons. Resuspending the sample in agarose prior to electrokinetic injection was instead suggested as a low cost method to preferentially reduce the concentration of long fragments during electrokinetic injection. This method was investigated at the Marziali lab and at the Genome Sciences Center and ultimately adopted as standard procedure on the MegaBACE 1000 at the GSC.

The GSC proved the agarose resupension technique by resuspending identical pooled and realiquoted samples from a single clone from the mammalian genome collection (MGC-10790) in $\mathrm{dH}_{2} \mathrm{O}$ and various concentrations of Agarose [58]. Sample preparation is described in detail above in Appendix A.5 Pairs of identical plates were resuspended in $20 \mu \mathrm{L} \mathrm{d0.02 \%} \mathrm{to} 0.6 \%$ (SeaKem Gold Agarose FMC Bioproducts). One plate from each pair was sequenced on each of the two MegaBACEs at the GSC. With agarose resuspension, average read lengths rose from 198 to 653 bp and "successful" runs, 
classified as more than 50 Phred20 bases, increased from $47 \%$ to $98.4 \%$ of reads.
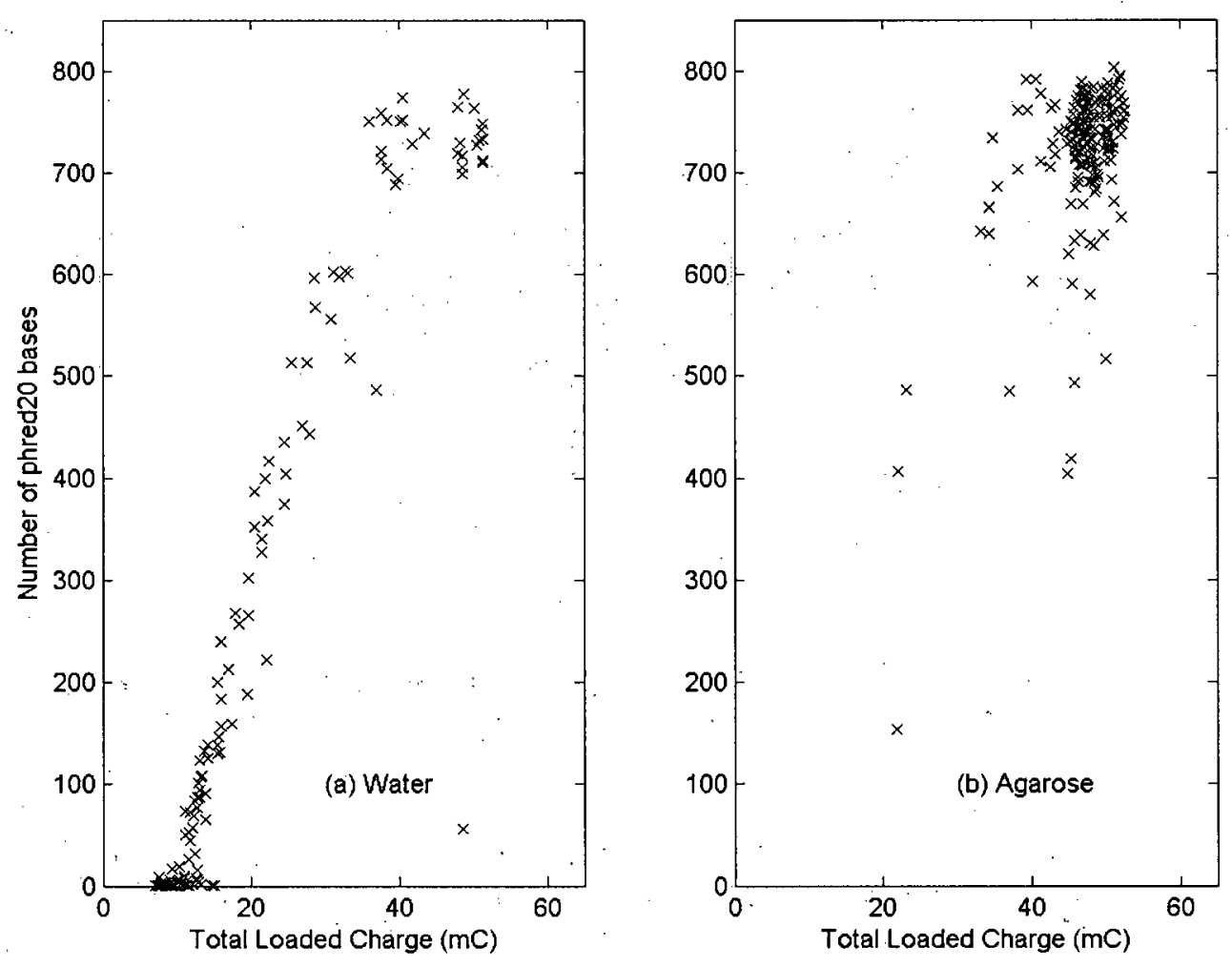

Figure 35 Read length vs total loaded charge for (a) Samples resuspended in $\mathrm{dH}_{2} \mathrm{O}$ as shown in Figure 8. (b) The same samples resuspended in $0.06 \%$ agarose. Each " $x$ " is one capillary.

The cause of the improvement with agarose was made clear by analyzing the raw current and signal data. Figure 35 compares total loaded charge after injection from water resuspension, (also shown Figure 8), with TLC after agarose resuspension. While in section 4.1 a correlation between TLC and read length was shown, the agarose almost completely prevented current decline from occurring. Almost all capillaries after agarose resuspension show a TLC over $40 \mathrm{mC}$. 
While the efficacy of loading from agarose was demonstrated, it was not known if the reduction in incidence of current decline was due to preferential suppression of large fragments or an overall suppression of fragments. This was investigated in the Marziali lab using the single capillary instrument. Samples of $1 \mathrm{~kb}$ ladder (New England Biolabs N3232S) consisting of 0.5 to $10 \mathrm{~kb}$ double stranded fragments was used as a sample with a representative size range, but with more a consistent injection behaviour then cycle sequence products, which likewise led to the choice of $\lambda$ DNA for current decline experiments. $20 \mu \mathrm{L}$ of ladder was mixed into $20 \mu \mathrm{L} 5 \times$ Sybr Green and

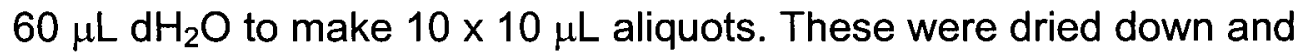

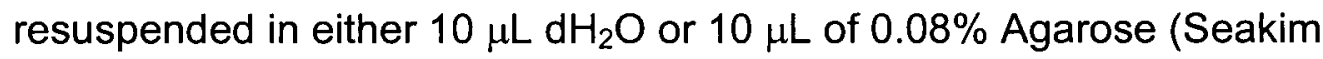
Gold, FMC Bioloabs) dissolved in $\mathrm{dH}_{2} \mathrm{O}$. The samples were then injected for $15 \mathrm{sec}$ at $5 \mu \mathrm{A}$ and run at $2 \mu \mathrm{A}$. Representative electropherograms are shown in Figure 36 . The ratio of the areas of the 3-10 kb peaks to the $500 / 517$ bp peaks for each electropherogram was then found. The average ratio for three samples resuspended in $\mathrm{dH}_{2} \mathrm{O}$ was 9.6 and the average for three samples resuspended in Agarose was 8.9, a difference of about $7 \%$. The reduction in peak area for the $500 / 517$ bp peaks were about $12 \%$ from water to agarose, and $18 \%$ for the $3-10 \mathrm{~kb}$ peaks from water to agarose. In other words, the overall reduction in injected DNA was larger than the preferential reduction in longer fragments. 


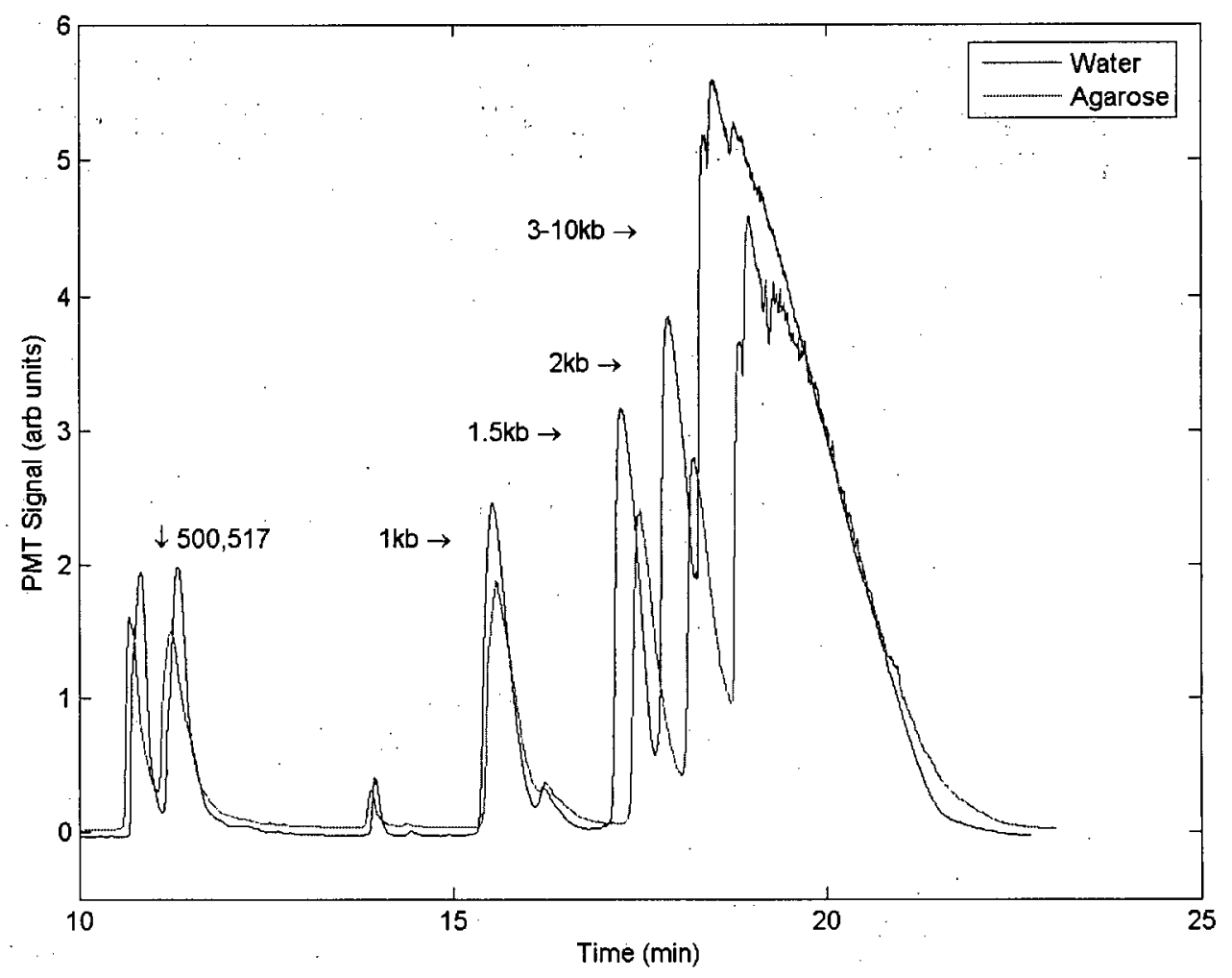

Figure 36 Electropherograms of $1 \mathrm{~kb}$ ladder resuspended in DI

\section{$\mathrm{H}_{2} \mathrm{O}$ and $0.08 \%$ Agarose}

Although long fragments could not be seen at the detector of the MegaBACE, the signal intensity of the first fragments could be measured. Data from the four plates shown in Figure 35b was analyzed in the following way. Capillaries with a total loaded charge over $30 \mathrm{mC}$, were isolated, as capillaries with lower TLC exhibited inconsistent florescence data. The flour channels of fluorescence data in the high TLC capillaries were summed and the first fifteen minutes of that data starting from the onset of peaks at the detector was integrated to get a signal strength value for each capillary. These were then averaged over all high TLC capillaries on that plate. The results, shown in Table 3, indicate that, the quantity of small fragments was 
reduced by about $20 \%$ by the agarose. The large variation in injected fragment quantity can also be seen in the range of values obtained.

\begin{tabular}{|l|l|l|l|}
\hline Machine & \multicolumn{2}{|c|}{$\begin{array}{c}\text { Integrated Fluorescence } \\
\text { Arb. Units }\end{array}$} & Drop \\
& $\mathrm{dH}_{2} \mathrm{O}$ & $0.06 \%$ Agarose & \\
\hline MB1 & $220 \pm 120$ & $164 \pm 75$ & $25 \%$ \\
\hline MB2 & $115 \pm 35$ & $98 \pm 21$ & $15 \%$ \\
\hline
\end{tabular}

Table 3 Signal strength of the first $~ 50$ DNA bases in capillaries resuspended in $\mathrm{DI}_{2} \mathrm{O}$ and Agarose. Machines refer to the two MegaBACE sequencers at the GSC.

Taken together, the MegaBACE and single capillary data for the effect of Agarose resuspension indicate that some preferential reduction in injection of large fragments is accompanied by a comparable reduction in the quantity of all fragments injected. This would tend to militate against the use of agarose in current sample preparation protocols even with a more sensitive but fault prone gel as dilutions have reduced the quantity of sample to near detection limits. (It was in fact found that the surface tension of liquid agarose made pipetting into 384 well plates impractical). The curious aspect of these results is that if agarose merely reduced the quantity of fragments across the board, the same result ought to be achievable by reducing injection time. This has never been reported as a viable option and it is not known why.

Perhaps the most plausible explanation is that the agarose works by preferentially retarding very long genomic fragments and that in fact these very long fragments are the primary culprit in current decline. The quantity of shorter $(<10 \mathrm{~kb})$ fragments may not be so critical in this matrix formulation, despite published reports in the past 
showing current decline to occur with template sized fragments. [14, 42]. 


\section{Appendix C Secondary Effects of Ionic Depletion and Bubble Behaviour.}

There are two effects which were observed in the course of this research which do not contribute to the main current decline effect, but should be of interest in understanding secondary current effects observed in capillaries. The first of these is the permanent effect depletion region formation has on the capillary. It is believed that a buildup of surface charge at the entrance to the capillary appears, which cannot be removed by replacing the matrix, and results in a mild depletion region formation every time the capillary is subsequently run. The second phenomenon is the rapid current decline occurring when the tube of matrix at the anode is replaced by buffer, as per the GSC's cost saving protocol. This is consistent with some positively charged species being removed from the matrix and is an example of the moving boundary equation in action. Finally, bubbles are revisited and it is shown how, despite bubbles not being the principle cause of major current decline, bubbles do contribute to the problem.

\section{C.1 Permanent Effects of DNA-Induced Depletion}

\section{Regions.}

It was observed that after formation of a DNA-induced depletion region, subsequent running of the capillary with new matrix and buffer would produce a depletion region at the cathode end without DNA or any other modifying agents. This phenomenon was found to occur consistently after the formation of a DNA-induced depletion region, but 
not after injection of DNA below the threshold for depletion region formation and not after the formation of depletion regions with depleted buffers. We tentatively call these "surface charge-induced depletion regions" (SCIDRs) as the formation mechanism survives matrix replacement so must be on the capillary surface, and it was shown in chapter 5 that the presence of bound charges is likely to be the cause of boundary movement of this type. A typical SCIDR is shown in Figure $37^{2}$.
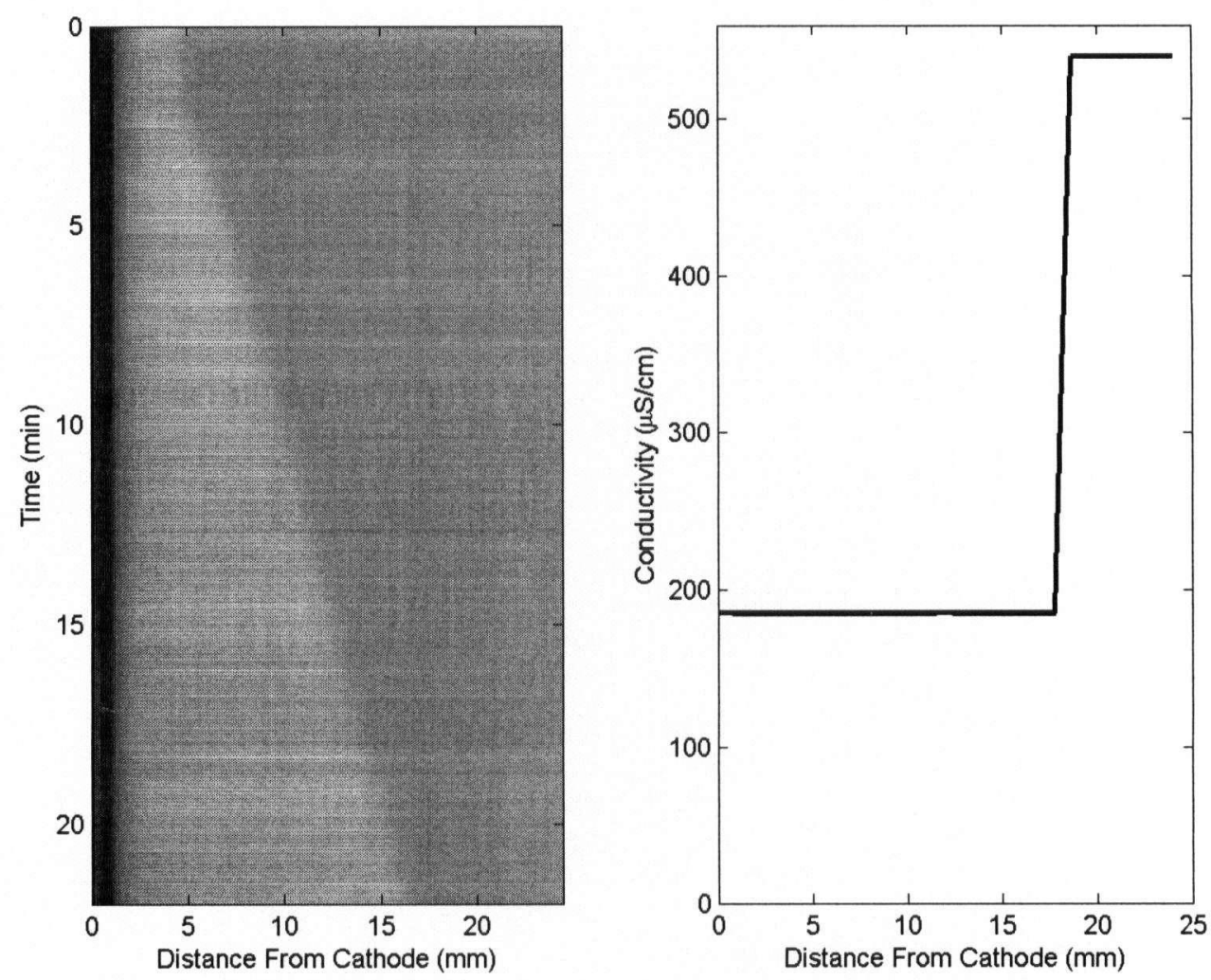

Figure 37. Infrared images (left) of a surface charge induced depletion region (SCIDR) propagating at $2 \mathrm{~m} / \mathrm{C}$. The conductivity profile (right), obtained by capillary cutting after the run finished, represents the capillary at the bottom edge of the left image at time $t=23 \mathrm{~min}$.

${ }^{2}$ If it is established that $\mathrm{pH}$ changes are the principle cause of boundary movement, these boundaries could be known as SpHIDRs! 
The driving mechanism for SCIDRs was found to be localized at the entrance of the capillary as shown in Figure 38. Here, $\lambda$ DNA and its depletion region were allowed to propagate into the capillary about $10 \mathrm{~mm}$. The matrix was then pumped out and the cathode buffer replaced. The subsequent SCIDR propagated right past the area affected by the DNA and depletion region. Neither the quantity of injected DNA nor the DNA's run time was found to correlate with the subsequent degree of SCIDR depletion or its propagation rate.

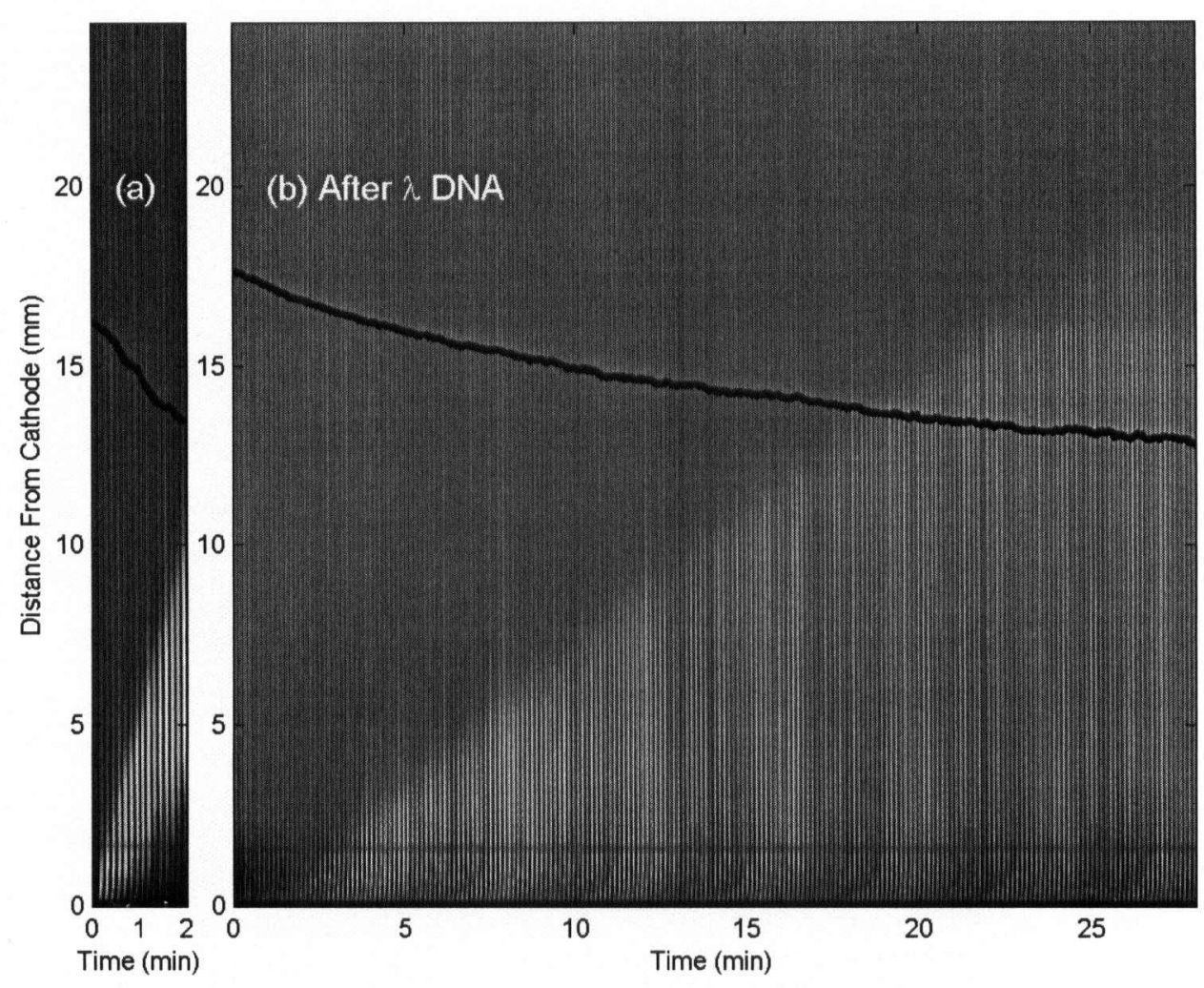

Figure 38 A fresh capillary with (a) $\lambda$ DNA injected to form a depletion region, and (b) running the same capillary with fresh LPA and cathode buffer. Current is shown, unscaled, in black. The depletion region in (b) propagates right past the area affected by the depletion region in (a). 
Even more direct evidence for changes at the capillary entrance being the source of SCIDRs is shown in Figure 39 where a SCIDR was allowed to grow to about $10 \mathrm{~mm}$. The first millimeter of capillary was then clipped off. The SCIDR boundary traveled forward another two millimeters and then came to a halt. When the LPA was again pumped out and electrophoresis restarted, no depletion region formed, which indicates that the part of the capillary causing the SCIDR had been permanently removed. This same experiment was repeated with DNA (not shown) where the IR image was used to locate the cathode boundary which was clipped off after five minutes. The anode boundary continued in another two millimeters and again came to a halt.
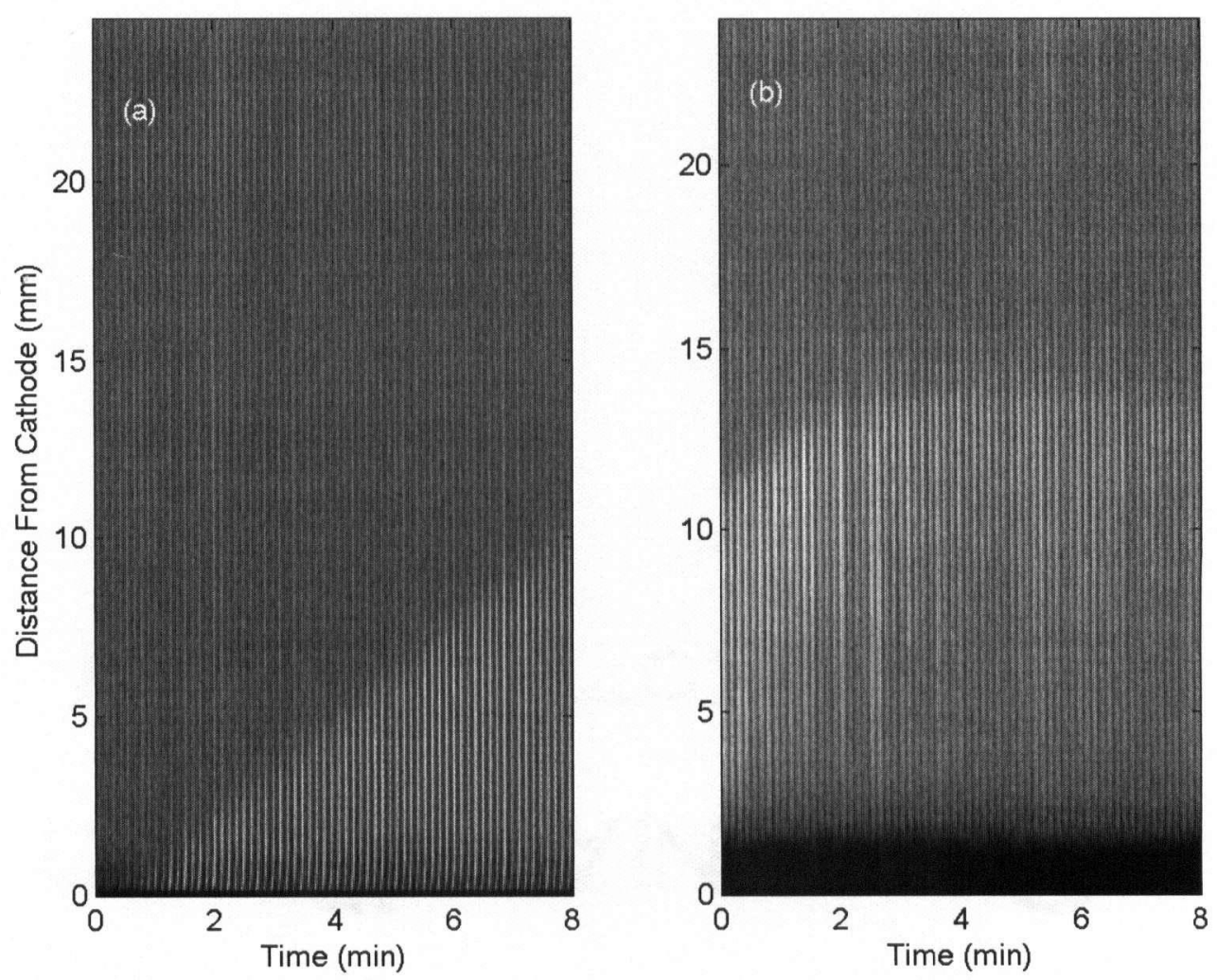

Figure 39 (a) A SCIDR advancing into the capillary at $2.1 \mathrm{~m} / \mathrm{C}$. (b) After clipping the first millimeter of capillary, the boundary advances at the same rate for a further $2-3 \mathrm{~mm}$ and then holds stationary. 
The observations described above are intriguing and shed some light on how SCIDRs may form as well as, more generally, how the anode boundaries may propagate. As this is a surface effect, it must involve interactions with the surface coating. The anti-EOF coating in these capillaries is made by replacing the easily hydrolyzed Si-O bonds at the surface with $\mathrm{Si}-\mathrm{C}$. These are then linked to acrylamide polymers which remain fixed in place at the wall and presumably interact with LPA as it is pumped in to prevent it from moving under EOF [48]. Deterioration of the coating is believed to manifest itself through some combination of shearing of the fixed acrylamide, hydrolysis of exposed Si-O groups and adsorbtion of charged species onto the capillary wall. The fact that SCIDRs are driven by some wall modification close to the entrance of the capillary suggests either a localized $\mathrm{pH}$ rise resulting in rapid hydrolysis or adsorbtion of DNA onto the capillary wall. It is not clear why either of these effects should occur only at the entrance of the capillary.

The fact that the boundaries come to a halt and the depletion regions remain fixed after the source of the depletion region is removed is to be expected. Once a change in concentration is established and the ionic species in each region are restored to the same proportions, the regulation function requires that the boundary remain stationary. That this boundary remains fixed also lends strong weight to the notion that the boundary is definitely not driven by a change in transference number as in equation (5-42).

The residual movement of the boundaries after the end of the capillary is clipped is interesting and the reasons why are not clear. If fixed charge was causing the boundary to move, it might be expected to keep moving as further fixed charge would continue to be exposed. If the fixed charge was induced by a change in $\mathrm{pH}$ on the capillary surface or in the acrylamide, the restoration of the $\mathrm{pH}$ would presumably neutralize this effect. If the SCIDR boundary is driven by a pure change in $\mathrm{pH}$, as described in section 5.4 .5 , the excess $\mathrm{OH}^{-}$ions in the depletion region will be swept out by TAPS $^{-}$ions coming in from the newly clipped end of the capillary. If the depletion region is of length $l_{d}$, the remaining 
$\mathrm{OH}^{-}$ions in the depletion region $\left[\mathrm{OH}^{-}\right]_{\mathrm{d}}$ can move the boundary forward a distance $I_{e}$ according to

$$
l_{e}=l_{d} \frac{\left[O H^{-}\right]^{d}}{\left[T_{A P S^{o}}\right]^{b}}
$$

where $\left[\text { TAPS }^{0}\right]^{b}$ is the neutral TAPS concentration in the background region. The depletion region conductivity is assumed to be $160 \mu \mathrm{S} / \mathrm{cm}$ and $\left[\mathrm{TAPS}^{\circ}\right]^{\mathrm{b}}=$ $25 \mathrm{mmol} / \mathrm{L}$. Equation (5-52) then gives the correct propagation rate if the depletion region's $\mathrm{pH}$ is 9.3 of $\left[\mathrm{OH}^{-}\right]=0.02 \mathrm{mmol} / \mathrm{L}$. This, however, gives a residual propagation distance $l_{e}$ of $7 \mu \mathrm{m}$, more than two orders of magnitude too small. While the nickel particle data suggests the $\mathrm{pH}$ does indeed rise, this datum suggests the $\mathrm{pH}$ rise itself is not the only mechanism moving the boundary. These conclusions are not necessarily contradictory: the actual rise in $\mathrm{pH}$ is not known and it may not take a large rise in $\mathrm{pH}$ to charge the nickel particles enough to change direction. A small rise in $\mathrm{pH}$, not enough to move a boundary, may be enough to charge the relevant species enough to display the observed behaviour.

Intuitively, the mechanism that best fits the data is that some $\mathrm{pH}$ change does occur which has the effect of locally charging the matrix or capillary wall. How this charge-induced $\mathrm{pH}$ change occurs and how the inside of the capillary is affected are unknown. This clearly represents an opportunity for significant future investigation. Future work could involve thermal imaging and capillary cutting as well as online monitoring of $\mathrm{pH}$ with UV detectors and indicator solutions as well as the setting up of artifical $\mathrm{pH}$ gradients and possibly different matrix polymers.

As an aside, it emerged in the course of this analysis, that the depth of single-sided depletion regions could be calculated from the rate of current decline, assuming a given background conductivity and consistent behaviour at the anode. This was done by fitting the current to the rising resistance: 


$$
I(t)=\frac{V}{R_{o}\left(l-x_{b}(t)\right)+R_{d} x_{b}(t)+R_{o f f}}
$$

where $R_{0}$ is the initial resistance/unit length, $I$ is the capillary length and $x_{b}(t)$ is the measured boundary position. The depletion region resistance $R_{d}$, and $R_{\text {off, }}$ an offset for the part of the capillary submerged in the buffer, are free parameters. For Figure $38 \mathrm{~b}$ this gives a depletion depth of $160 \mu \mathrm{S} / \mathrm{cm}$, similar to that observed for the cut capillary in Figure 37. This method is only useful when there is only one depletion region and the rest of the capillary is electrically stable. In such circumstances it can be used to nondestructively estimate the depletion region conductivity.

\section{C.2 Matrix ion depletion from anode-side buffer.}

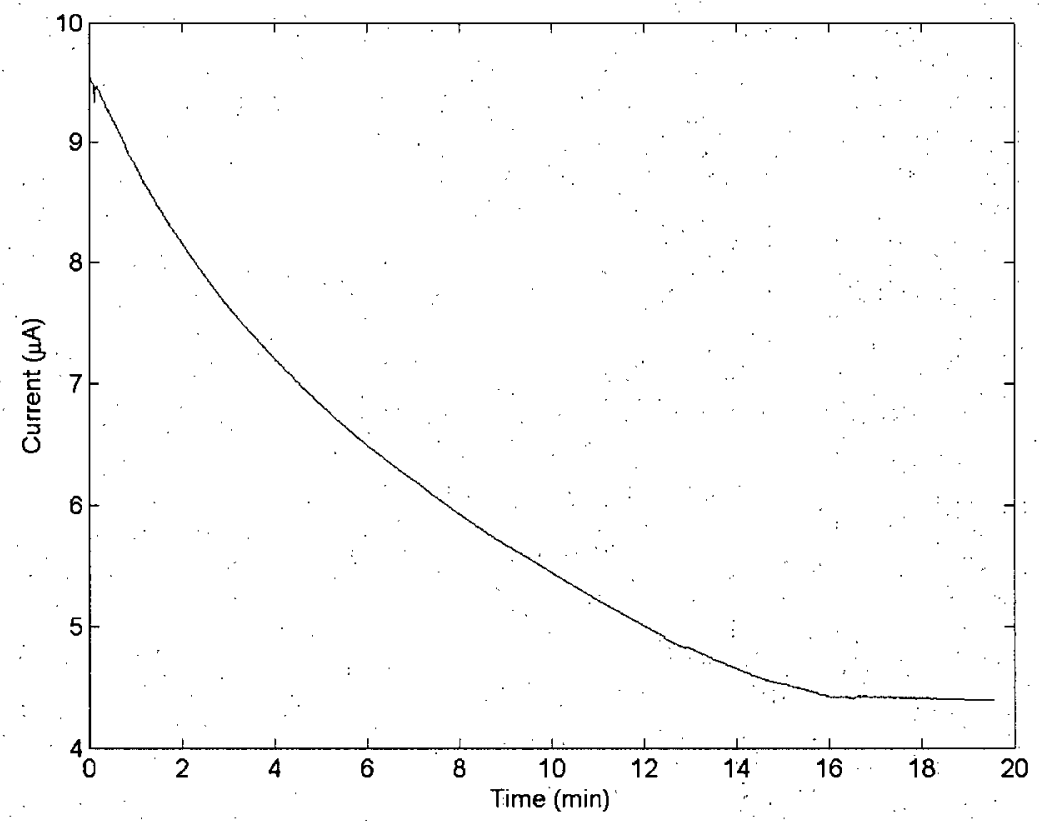

Figure 40: Typical current decline associated with depleted matrix for a $36 \mathrm{~cm}$ capillary after replacing the LPA matrix at the anode with aqueous buffer and applying 5000V. 
As discussed in section 3.1, it was observed that replacing the LPA at the anode end with buffer would cause the current to decline by about $50 \%$, and then stabilize. A typical example of this highly repeatable current decline is shown in Figure 40. Assuming a sharp moving boundary between regions of different conductivity, a propagation rate was calculated from the time taken from the beginning of the run to when the current stabilized. This corresponded to a propagation rate of $75 \mathrm{~m} / \mathrm{C}$. A capillary was then cut up from the anode end after four minutes run time and a boundary was indeed found at the location predicted by the propagation rate. The anodeside conductivity was typical for that of undepleted capillaries and the cathode-side conductivity was typical of that for completely depleted capillaries.

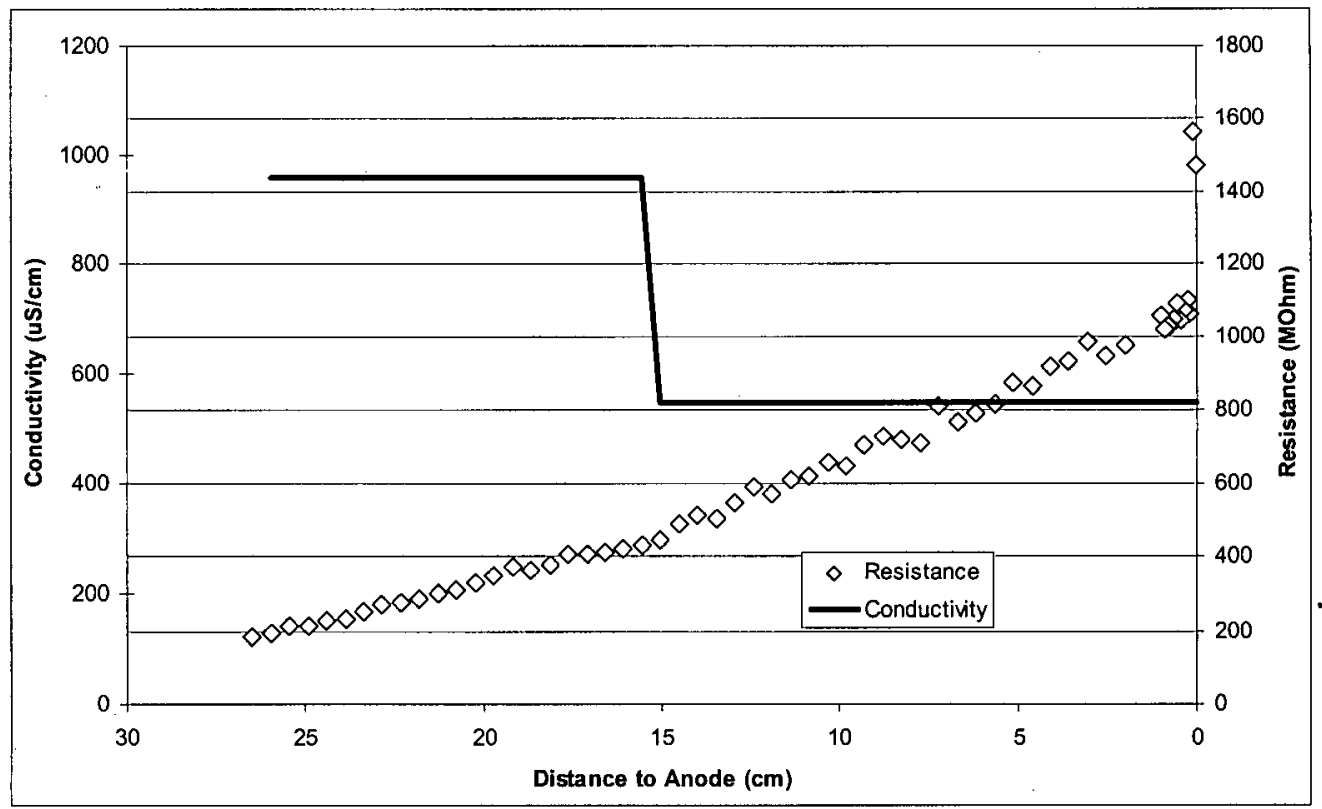

Figure 41 Cut capillary data from four minutes into matrix depletion. The anode and cathode sides of the boundary are 958 and $545 \mu \mathrm{S} / \mathrm{cm}$ respectively. Note that this change in conductivity is associated with a very subtle change in the slope of the resistance curve. 
In ignorance of buffer and matrix components, it is not possible to say exactly what process is occurring in the capillary. The moving boundary equations and regulation function, however, can give some insight into the properties of the moving ions. This self sharpening boundary must be propagating at the speed of the leading ion. This rate of $75 \mathrm{~m} / \mathrm{C}$ in the $1200 \mu \mathrm{S} / \mathrm{cm}$ background electrolyte corresponds to $40 \times 10^{-9} \mathrm{~m}^{2} \mathrm{Ns}$. This is faster than Tris and more typical of small positive ions like $\mathrm{Na}^{+}$. This suggests that an ion left over from the polymerization of the LPA is being removed [89]. The situation that is believed to arise is similar to that shown in Figure 25 where there is a fixed boundary at the anode entrance to the capillary defined by the regulating function, and a moving boundary with different ionic species on either side. This explains the experimentally observed result that increasing the buffer concentrations at the anode doesn't change the capillary conductivity. As noted, it is not known what ion is being removed from the matrix, although it is possible that it is excess TEMED [47]. TEMED is basic, so would produce a positively ionic species. In fact, equations (5-35) and (5-39) can be solved numerically for the concentrations of Tris and the unknown ion in the undepleted matrix. If the concentration of Tris $^{+}$and TAPS ${ }^{-}$is assumed to be 25 $\mathrm{mmol} / \mathrm{L}$ on the depleted side, and given mobilities in Table 2 , the dissociated $\left[\mathrm{Tris}^{+}\right]=5 \mathrm{mmol} / \mathrm{L}$ and [unknown ion] $=24 \mathrm{mmol} / \mathrm{L}$ on the undepleted side. If the LPA had started with $50 \mathrm{mmol} / \mathrm{L}$ Tris/TAPS these concentrations of undissociated species could easily arise in an excess of a positively charge free ion and would give a $\mathrm{pH}$ of 8.7 . In about $75 \%$ of cases where matrix depletion was recorded, two abrupt transition points were visible at the end of the period of current decline. These are shown, along with what is believed to be their cause, in Figure 42. The first point corresponds with the matrix depletion boundary from the anode crossing a SCIDR boundary from 
the cathode. The second point corresponds to the matrix depletion boundary exiting the cathode end of the capillary. The IR image shows the SCIDR boundary speeding up from 1.7 to $3.1 \mathrm{~m} / \mathrm{C}$ as the invisibleto-infrared matrix depletion boundary comes past. This is to be expected from equation (5-52) as conductivity drop from (typical values of) 1100 to $600 \mu \mathrm{S} / \mathrm{cm}$ would predict exactly such a rise. The DNAinduced depletion region adjusts its propagation rate in the same way, shown in Figure 42(b). The anode-side of its depletion region propagates across the SCIDR at $68 \mathrm{~m} / \mathrm{C}$, then slows to $16 \mathrm{~m} / \mathrm{C}$, a typical rate in depleted matrix. Inspection of all the matrix depletion data showed that cases where these transitions were observed always corresponded to cases where $\lambda$ DNA had been previously introduced. Fresh capillaries did not show this behaviour. 

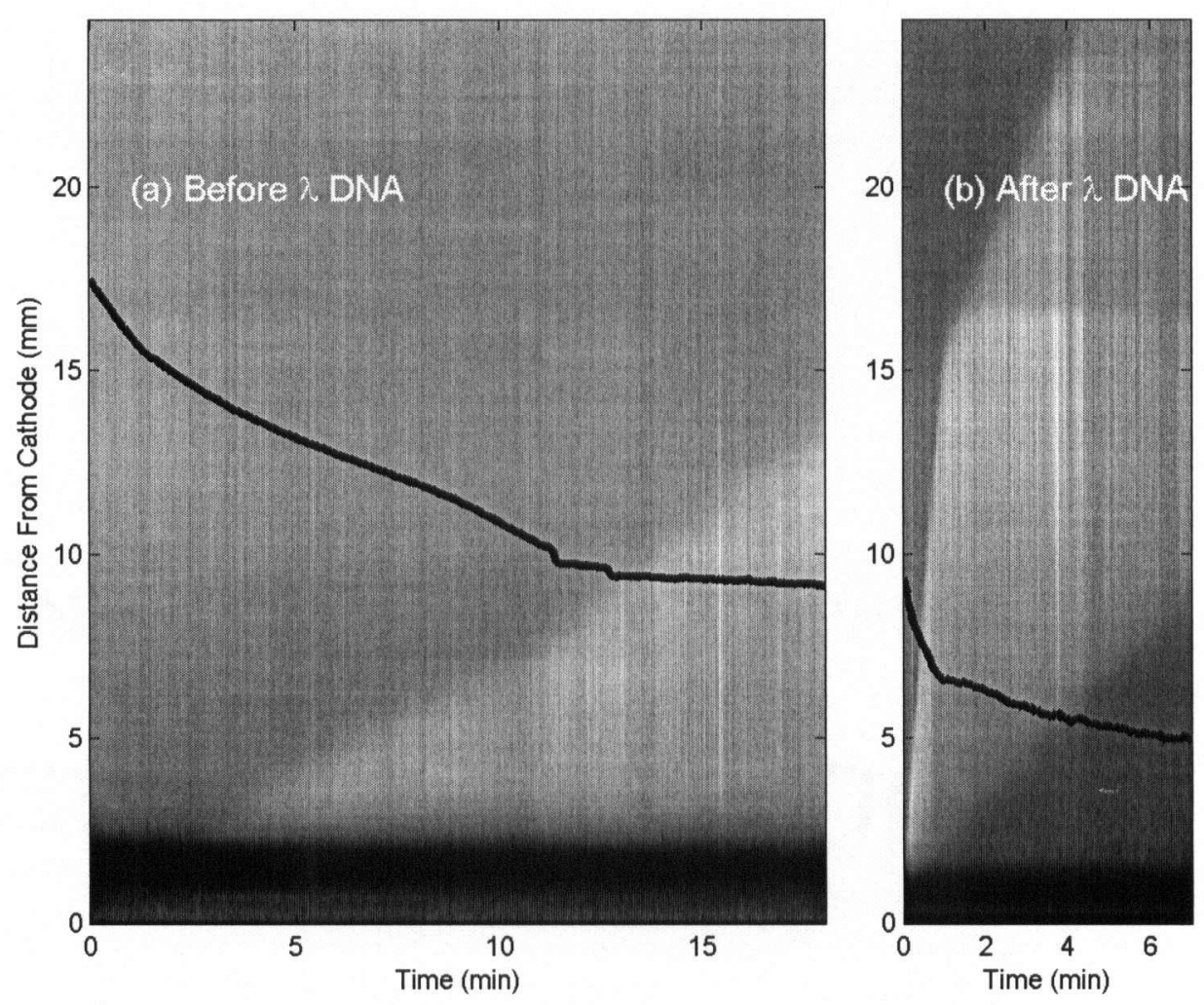

Figure 42 (a) Thermal images of a surface charge induced boundary crossing an invisible-to-IR depleted matrix boundary. The upward traveling SCIDR boundary initially propagates $1.7 \mathrm{~m} / \mathrm{C}$, rising to $3.1 \mathrm{~m} / \mathrm{C}$ as it crosses the downward traveling depleted matrix boundary. Note the change of slope of the thermally imaged depletion region at $\mathbf{t}=$ $11 \mathrm{~min}$ in (a). Current, falling from $9 \mu \mathrm{A}$ to $5 \mu \mathrm{A}$, is shown in black. (b) After the DNA is injected, the anode boundary races to the edge of the SCIDR boundary at $68 \mathrm{~m} / \mathrm{C}$ where it slows to $16 \mathrm{~m} / \mathrm{C}$.

\section{C.3 Bubbles' Effect on Current}

It was found that although bubbles are not a primary source of current decline, they do act to perturb current decline and affect depletion region growth. The relationship between bubble and 
depletion region growth was examined in detail with thermal and visible light imaging combined with capillary cutting. Bubbles were introduced by capillary cooling and then $\lambda$ DNA was injected. Figure 43 shows a representative example. While bubble growth occurs with the depletion region, the depletion region actually grows faster than the bubble, while the bubble appears to be driven forward with the cathode boundary. This was typical of continuous long bubbles in the single capillary instrument.
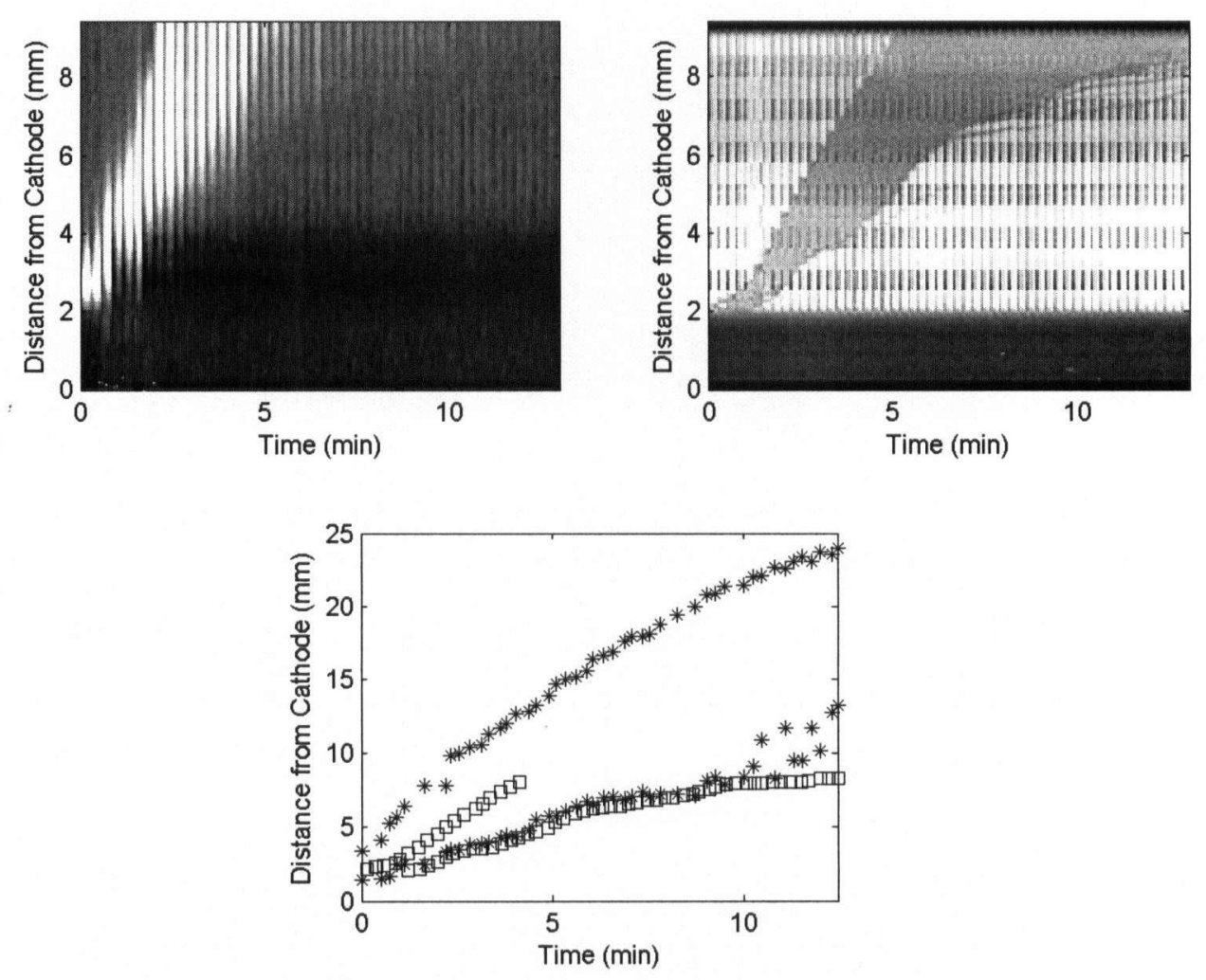

Figure 43: Infrared (top left) and visible (top right) images of the capillary showing simultaneous growth of a hot region and bubble. The positions of the two are superimposed below, showing the hot region advancing substantially ahead of the bubble. The depletion region boundaries are shown with (*) and the bubble boundaries with ( $\square$ ) 


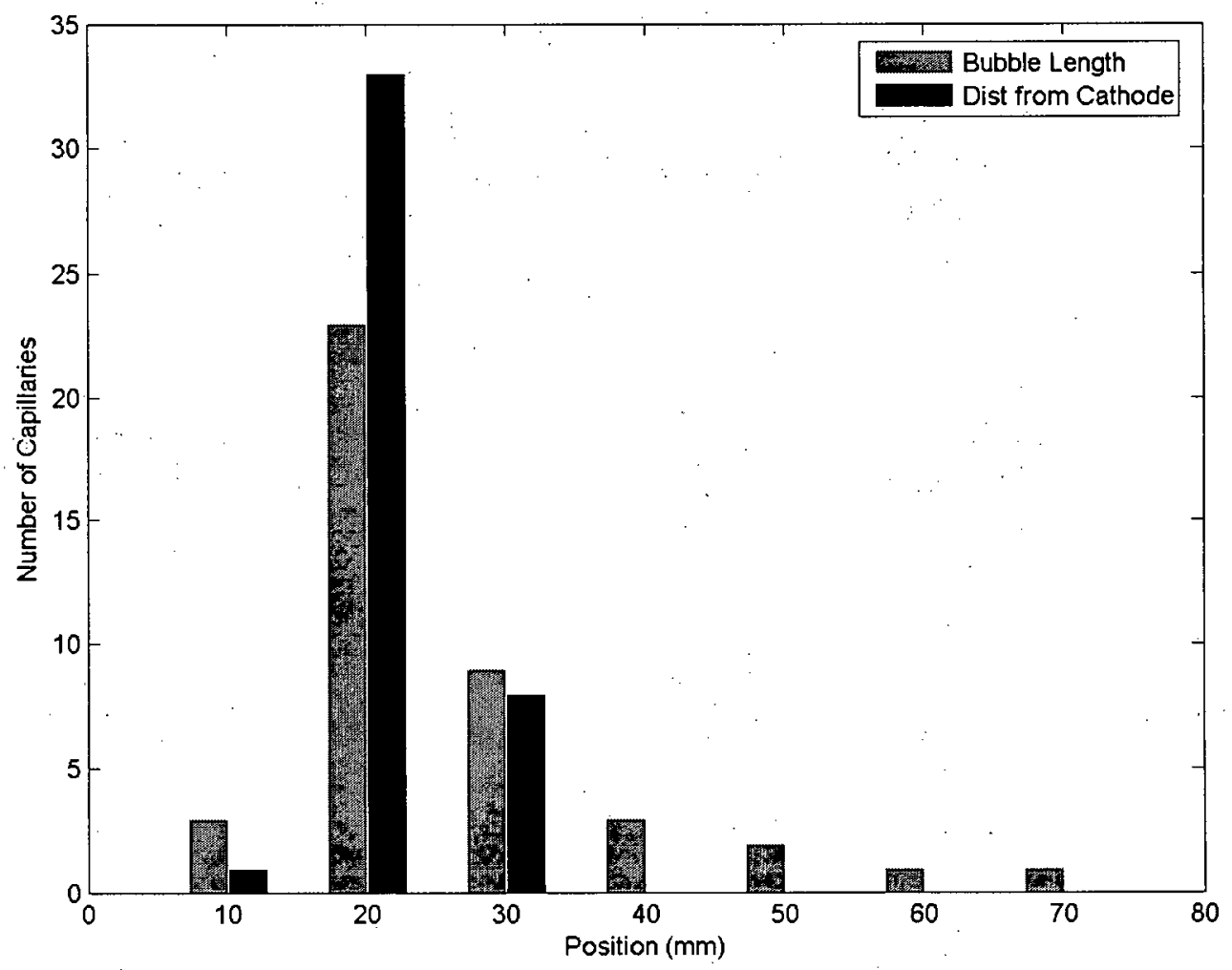

Figure $44 \mathrm{~A}$ histogram showing observed bubble positions after one run. The bubble's cathode ends line up with the expected location of depletion regions seen in bubble-free measurements, but the bubbles

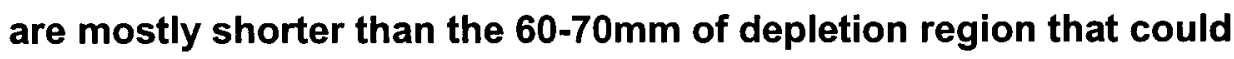
account for the observed current.

Indirect evidence of the same behaviour is seen in the MegaBACE. Figure 44 shows data from one plate where continuous long bubbles were isolated and binned by cathode end location and length. Most bubbles begin 25-30 mm into the capillary and are 25 to $30 \mathrm{~mm}$ long. The bubbles' cathode ends are approximately coincident with cathode side boundaries of ionic depletion regions observed with the single capillary instruments for runs of comparable total loaded charge. These bubbles are, however, considerably shorter than the 
$60-80 \mathrm{~mm}$ ionic depletion regions at $2-5 \%$ of the background conductivity required to produce the observed current in the single capillary experiments. These $20-30 \mathrm{~mm}$ long bubbles would have to have a surrounding conductivity of about $1 \%$ of the background electrolyte, lower than any measured depletion region. We conclude therefore that Figure 44 shows the same effect as Figure 43, and the depletion region expands faster than the bubbles and that the bubbles are definitely not the cause of current decline even where they are well developed.

Although bubbles are clearly not the principle cause of current decline, they do manifest themselves in the current data in the form of current variability. Consider again the two plates shown in Figure 8, page 44, and Figure 35(a), page 135. These were called plate 4105 and plate 4106 in the GSC's records and will henceforth be together called $410 x$. In single capillary experiments, the rate of current decline in bubble-free runs was very repeatable, as shown in Figure 11 and Figure 12. If such behaviour was observed in the MegaBACE, one would expect the $410 x$ plates to show a bimodal distribution, with all capillaries showing either no decline and TLC $50 \mathrm{mC}$ or current decline and TLC $10 \mathrm{mC}$. Figure 45 bins the $410 x$ plates' TLC by frequency. There is biomodal behaviour, with peaks at 10 and $50 \mathrm{mC}$, but there are a substantial number of capillaries with intermediate charge as well. These are capillaries where bubble growth is causing some degree of current decline without the existence of a depletion region. 


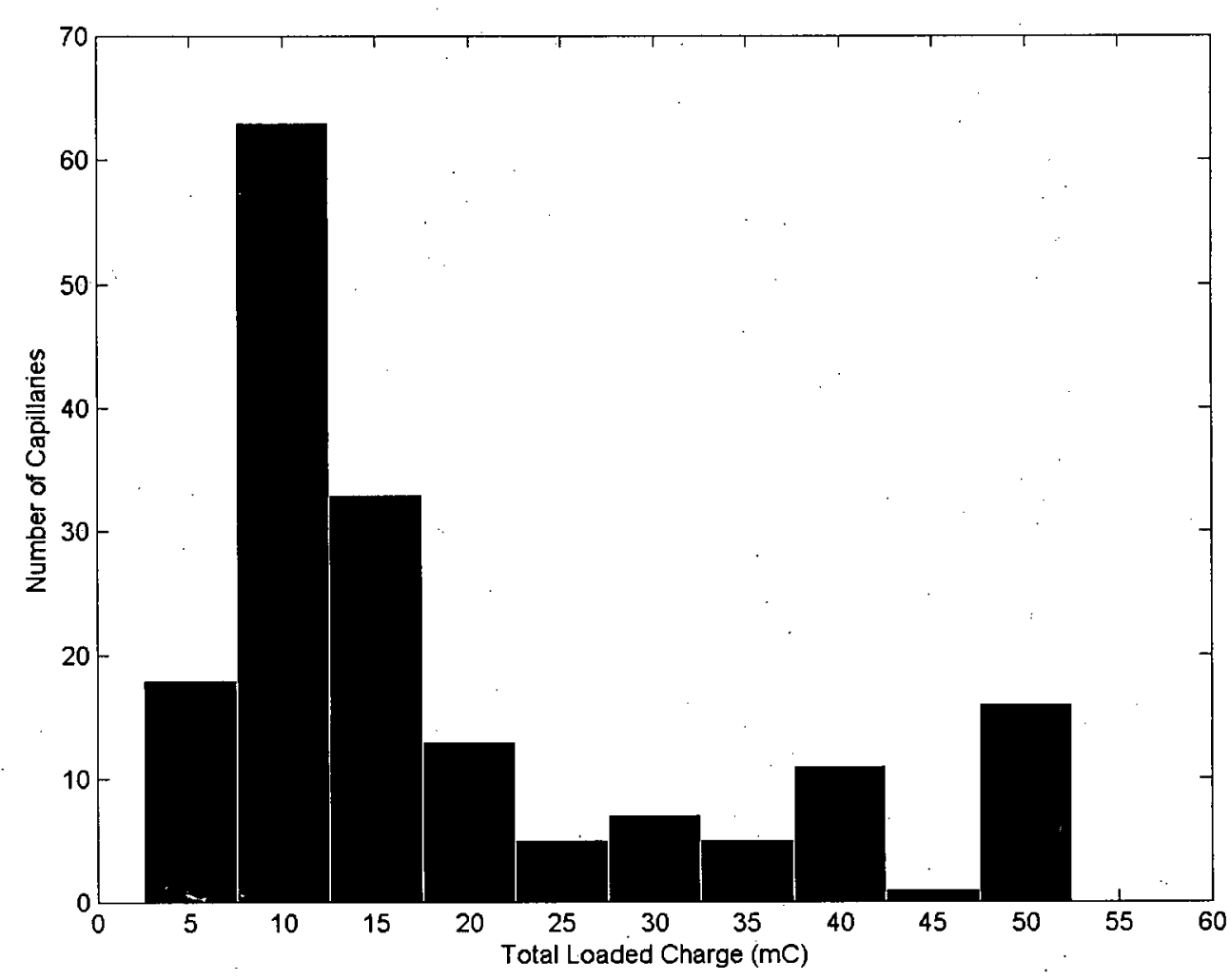

Figure 45. A histogram of the data in Figure 8(a), binning the capillaries by total loaded charge. Some bimodal behaviour is observed.

The source of these intermediate degrees of current decline can be seen in Figure 46, which shows current traces for the 4105 plate. A menagerie of unusual behaviours is seen. D07 and E10 show the current declining for a time and then rising. A06 and B04 show high frequency dips early on while in A08 the dips persist. D09 shows a profile consistent with bubble-free decline. Column 06 shows various capillaries which experience about $25 \%$ decline. High frequency (on the order of minutes or sections) variations have been reported elsewhere [42] and are consistent with our single capillary observations of artificially introduced bubbles. It may be that local heating temporarily pinches off the current, causing it to fall, which in 
turn cools the capillary and shrinks the offending bubble. Capillaries such as $\mathrm{C} 02$ which experience steady decline at a lower rate are consistent with "bubble trains" seen in the MegaBACE and single capillary instrument where one bubble propagates many centimeters into the capillary, leaving a string of small bubbles behind it at regular intervals. These could generate enough resistance to cause current decline but at a rate lower than that associated with the growth of a full blown depletion region.

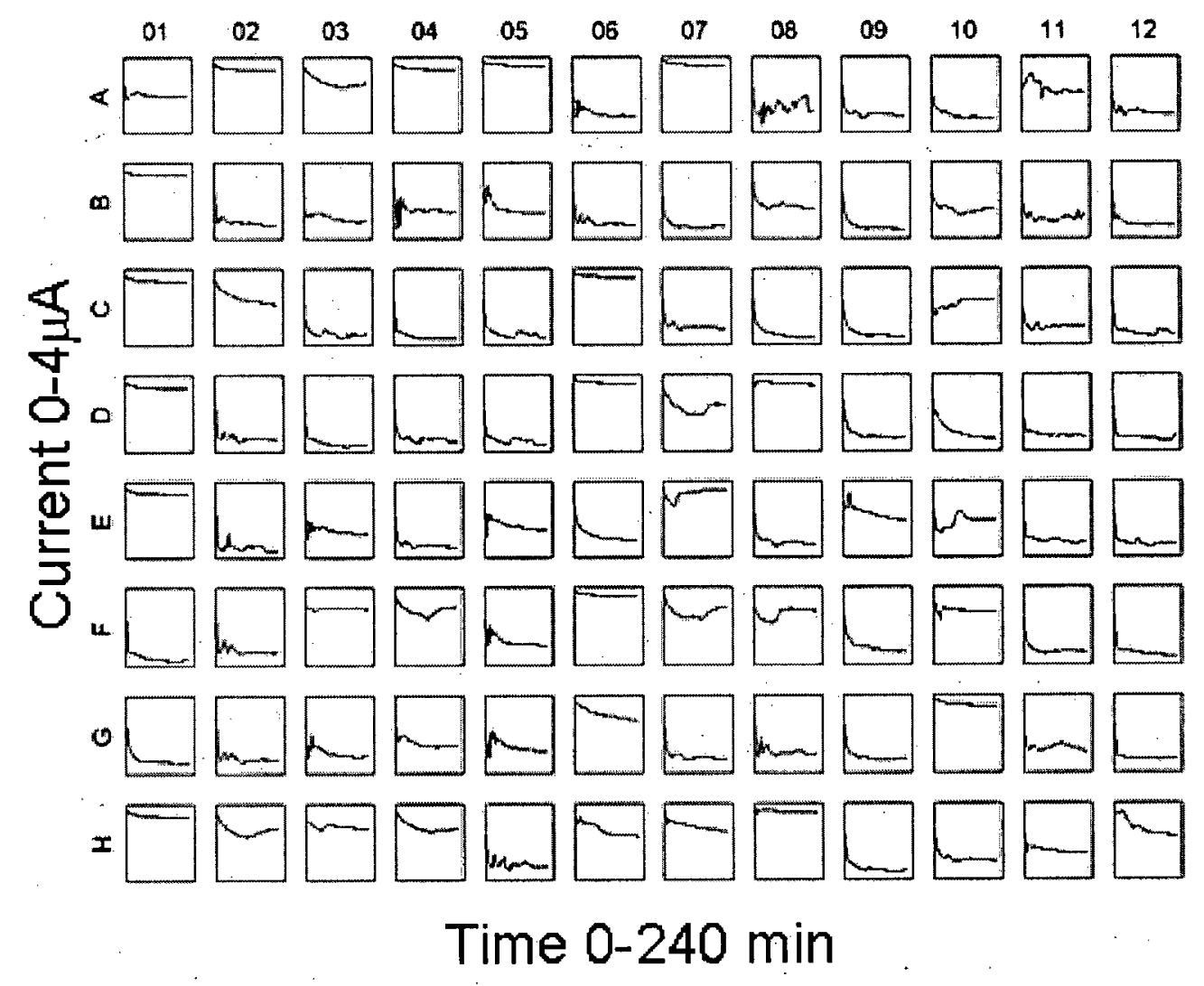

Figure 46 Current traces from one plate from the MegaBACE. The $Y$-axes are current $(0-4 \mu \mathrm{A})$ and the $X$-axes show time (0-240 minutes). For a description of bubble types see text. 


\section{Appendix D LPA and Buffer Properties}

\section{D.1 Conductivity and pH of Tris/TAPS Buffer Under Dilution}

For various reasons, it was necessary to measure the conductivity and $\mathrm{pH}$ of the buffer under different temperatures and dilutions. First, no value of the mobility of the TAPS ion was to be found in the literature. Second, the effect of buffer depletion on moving boundaries (section 5.4) or charged particles (section 5.4.4) would be mediated by a change in molar conductivity $(\sigma / \mathrm{C})$ and/or $\mathrm{pH}$.

$1.2 \mathrm{~g}$ of Tris and $2.4 \mathrm{~g}$ of TAPS were dissolved in $200 \mathrm{~mL} \mathrm{dH_{2 }} \mathrm{O}$ to give a $50 \mathrm{mmol} / \mathrm{L}$ solution. The conductivity and $\mathrm{pH}$ were measured using a WWR Model $8000 \mathrm{pH}$ meter and a WWR Bench/Portable conductivity meter. The solution was then diluted by factors of two. The concentration of the dissociated ions was then calculated using the chemical equilbrium algorithm described in section 5.2 and fit to the observed conductivity data to find the mobility for TAPS. $\mu_{\text {TAPS }}$ was found to be $25 \times 10^{-9} \mathrm{~m}^{2} \mathrm{Ns}$. 

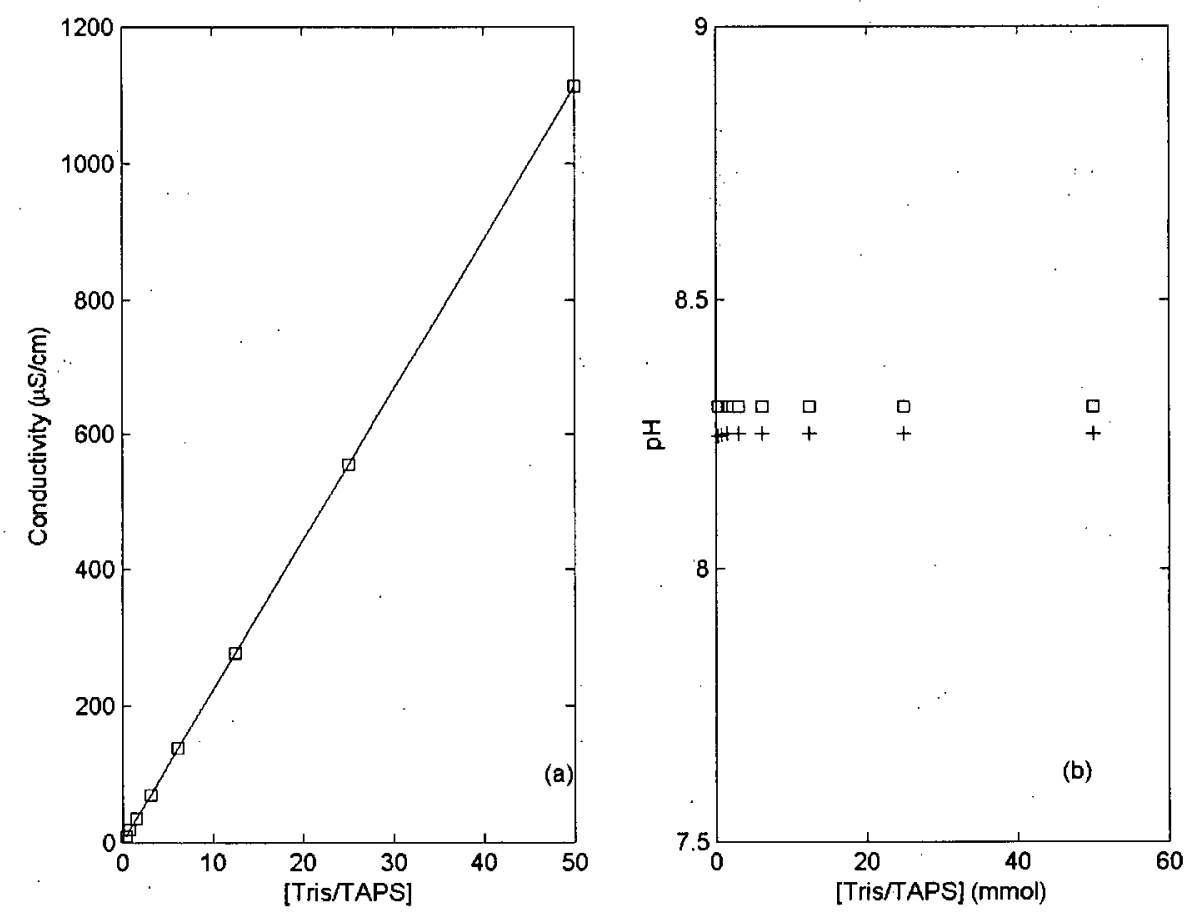

Figure 47 (a) ( $\square$ ) measured conductivity data fit with $\mu_{\text {TAPS }}=$ $25 \times 10^{-9} \mathrm{~m}^{2} / \mathrm{vs}$. (b) measured ( $\square$ ) and calculated (+) $\mathrm{pH}$ values.

The striking feature of the data in Figure 47 is the linearity of the conductivity over two orders of magnitude of concentration. This is different from the behaviour of most strong electrolytes, whose ions obey the Onsager limiting law near $1 \mathrm{mmol} / \mathrm{L}$ [87].

$$
\mu=\mu_{o}+A \sqrt{C}
$$



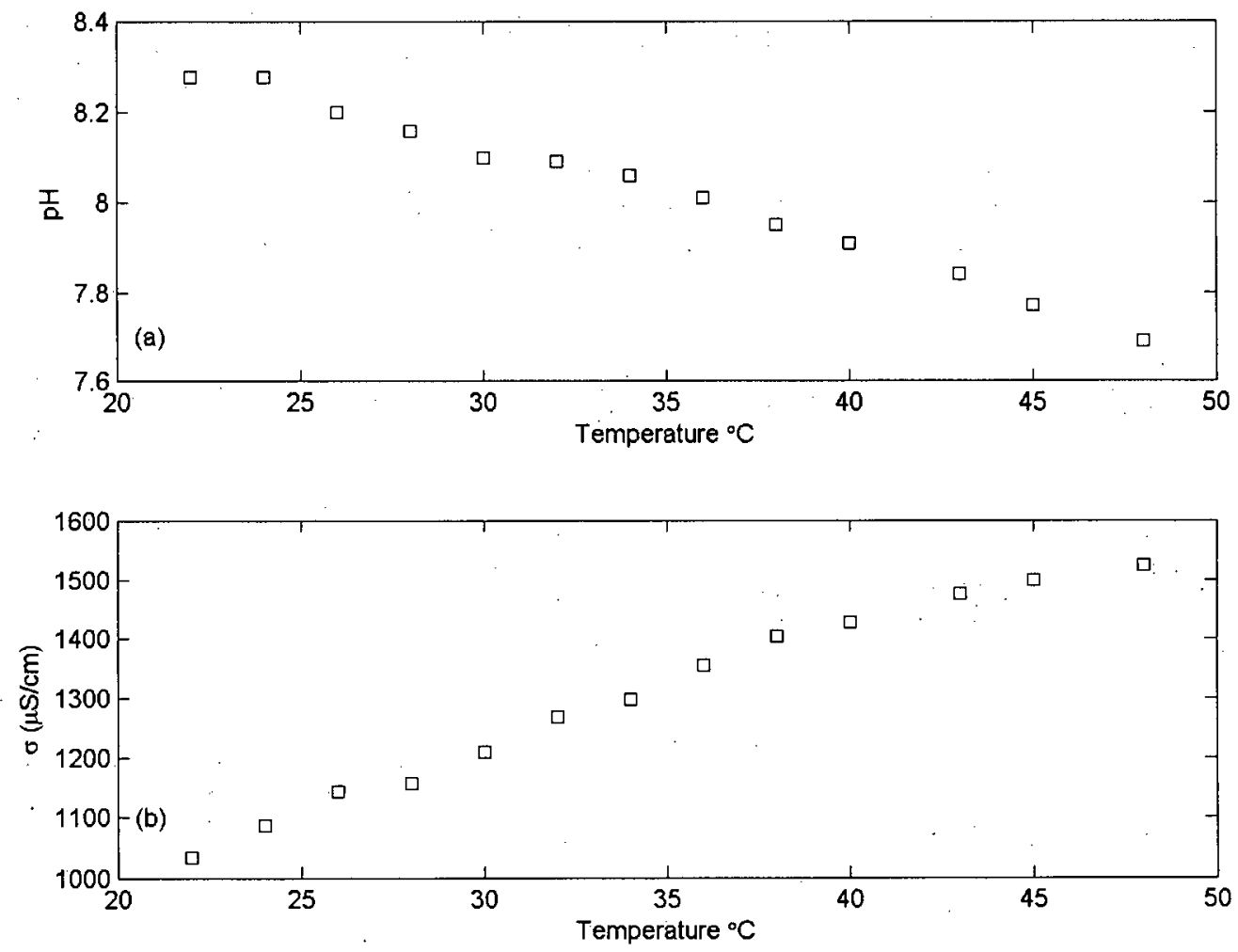

Figure 48 The temperature dependence of (a) $\mathrm{pH}$ and (b) conductivity in $50 \mathrm{mmol} / \mathrm{L}$ Tris-TAPS. $\mathrm{dpH} / \mathrm{dT}=-\mathbf{0 . 0 2}$ and $\mathrm{d} \sigma / \mathrm{dT}=0.02$.

Figure 48 shows the temperature variation of $\mathrm{pH}$ and conductivity of the Tris/TAPS. The former shows that the $\mathrm{pH}$ change is relatively small over the range of temperatures seen in this work (up to $7^{\circ} \mathrm{C}$ above ambient). The relationship between conductivity and temperature shows the aforementioned $2 \% /{ }^{\circ} \mathrm{C}$ variation discussed in the text.

\section{D.2 The Thermal Expansion Coefficient of LPA}

The thermal expansion coefficient of LPA was measured to test the hypothesis that matrix shrinkage due to cooling could cause bubbles to form in capillaries. This is discussed in section 4.2.2. LPA, 
which is about $95 \%$ water, was, surprisingly, found to have a thermal expansion coefficient almost twice that of water. Coefficients for water and LPA were measured by heating a filled capillary in the single capillary instrument, removing the anode and cathode buffer wells, and then removing the capillary. The distance to the resulting menisci at each end of the capillary was measured, and the coefficient of thermal expansion calculated from

$$
\alpha=\frac{\Delta L}{\Delta T} \frac{1}{L}
$$

where $L$ is the portion of capillary $(200 \mathrm{~mm})$ subject to temperature change. In this case the temperature changed from $50^{\circ} \mathrm{C}$ to $18^{\circ} \mathrm{C}$, resulting in the $\mathrm{dH}_{2} \mathrm{O}$ shrinking $2 \mathrm{~mm}$ and the LPA shrinking $4 \mathrm{~mm}$. The thermal expansion coefficients were respectively: $\alpha=2.7 \times 10^{-4}{ }^{\circ} \mathrm{C}^{-1}$ for water and $\alpha=5.2 \times 10^{-4}{ }^{\circ} \mathrm{C}^{-1}$ for LPA. In fact, $\alpha$ for water is irregular and falls from $0-4^{\circ} \mathrm{C}$, is 0 at $4^{\circ} \mathrm{C}, 2 \times 10^{-4}$ at $20^{\circ} \mathrm{C}$ and $4 \times 10^{-4}$ at $40^{\circ} \mathrm{C}$. It is probably reasonable to assume that LPA has twice the thermal expansion coefficient as water over the range of $20-40^{\circ} \mathrm{C}$. The average value of $\alpha$ for LPA in the temperature range of interest is therefore taken to be $3.8 \times 10^{-4}{ }^{\circ} \mathrm{C}^{-1}$ 


\section{Appendix E Temperature}

\section{Measurement}

It was initially thought that the actual temperature of the capillary might have a significant role to play the propagation rate of depletion regions. To that end, the temperature of the capillary was measured, first by thermal imaging with a reference temperature and then by direct measurement using a thermocouple bonded to the capillary. While it has been shown that temperature effects were unlikely to play a major role boundary movement, these infrared and thermocouple methods can be checked against literature values

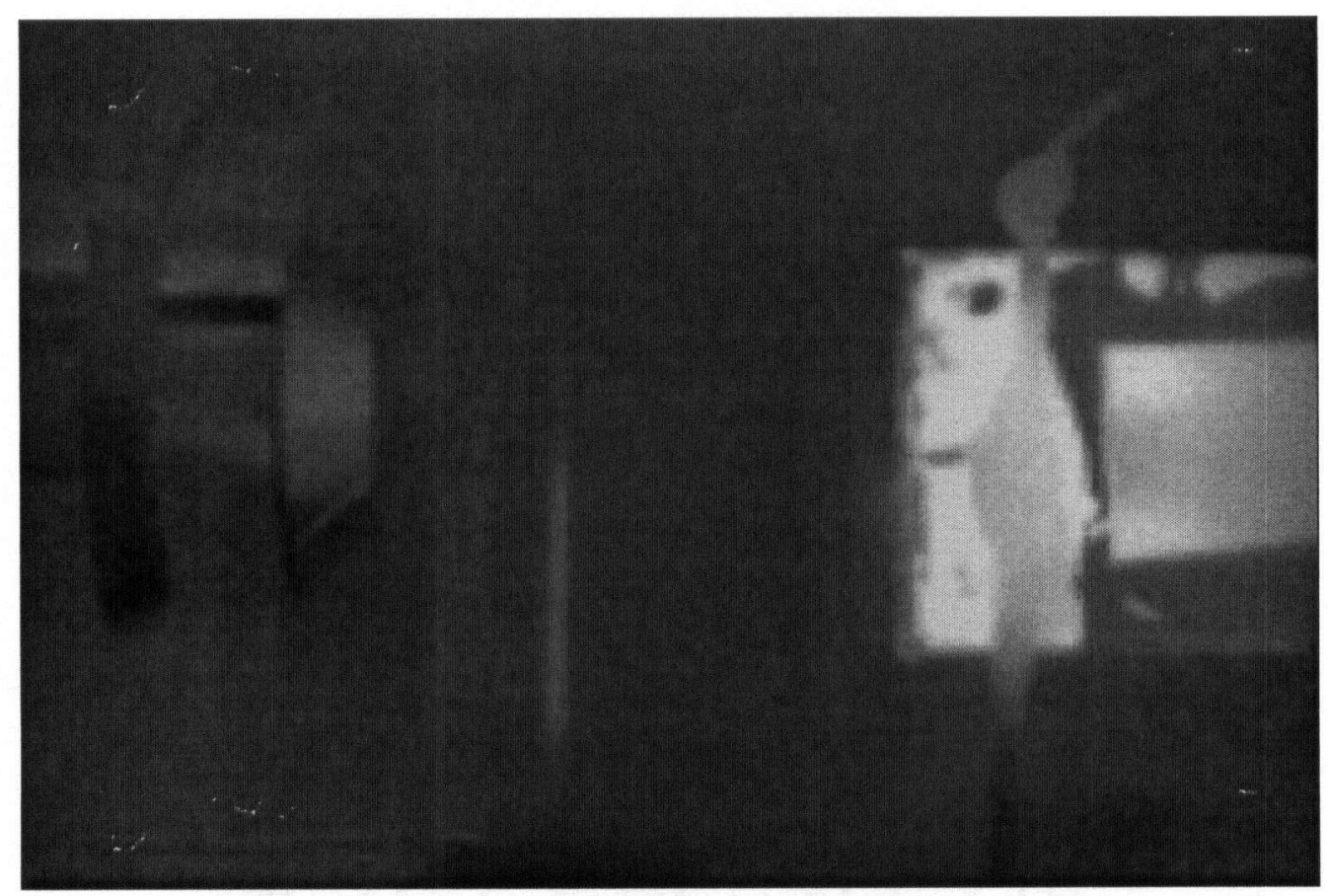

Figure 49 A still image from the infrared camera showing the LIF objective on the left, the capillary with the region of elevated temperature at center, and the reference heater on the right. The latter has a piece of capillary glued to an aluminum strip, with thermocouples located top and center. 
Figure 49 shows the capillary with hot region, microscope objective on the left and reference temperature device on the right. The latter has a piece of capillary glued with epoxy to an aluminum holder. On the back side of the aluminum, a loop of nichrome wire was glued down with a thermocouple (Omega Type T) bonded in the center. Another thermocouple was glued to the end of the capillary as seen at the top right of the image. These two data points were then mapped to the apparent brightness of the reference capillary to create a brightness vs. temperature map. The temperature of the actual capillary and depletion region was then calculated from that map. This had to be done for each image as the brightness adjustment of the thermal camera was automatic. For direct measurement, a 0.002" thermocouple (Omega Type T copper-constantin) was bonded to the capillary opposite the microscope objective. 

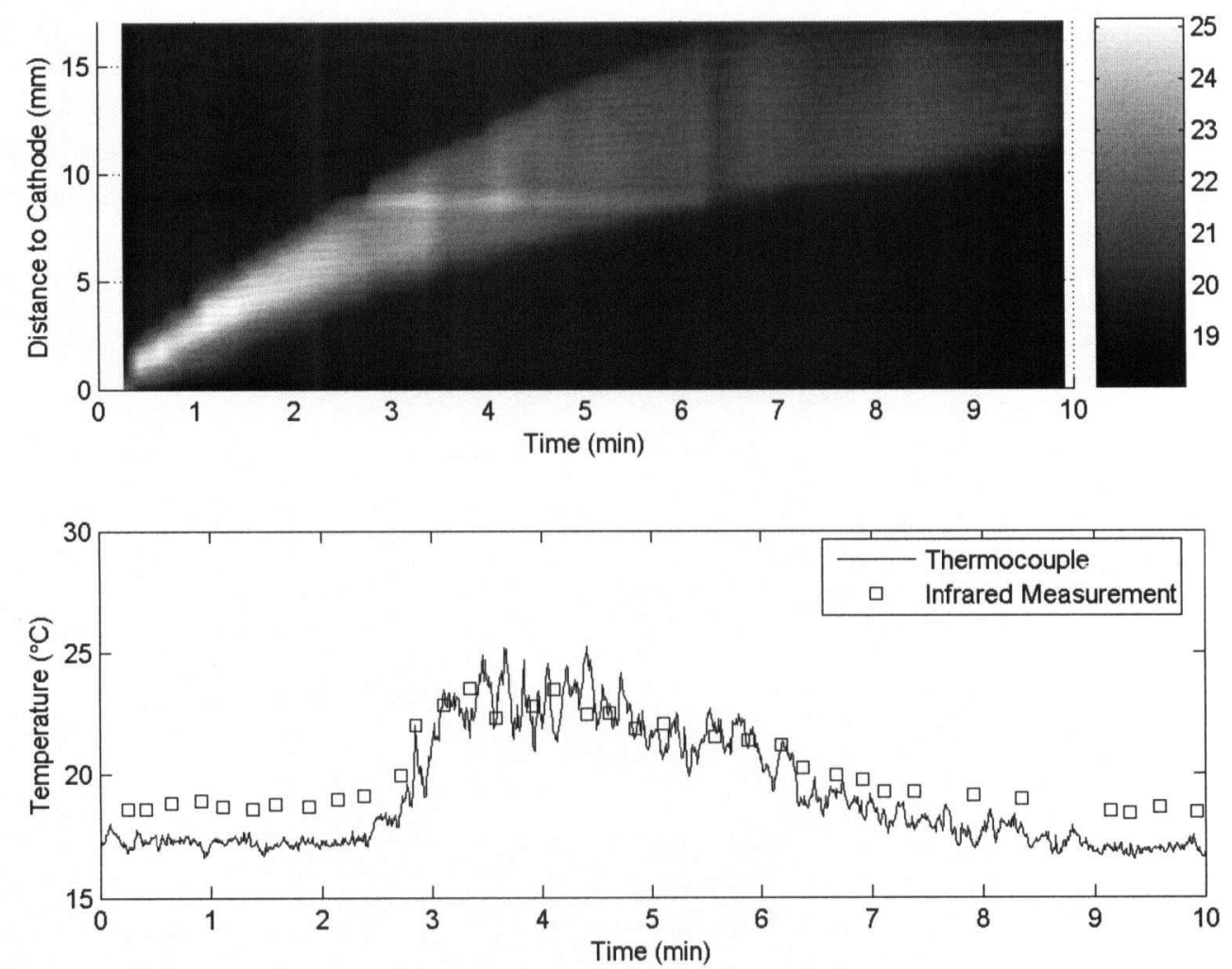

Figure 50 Top: A thermal image with temperature range shown in upper right. The thermocouple is visible as a while streak at across the IR image. Bottom: A comparison of the thermal image and thermocouple temperature data.

Both the thermocouple and thermal camera show a rise in temperature of about five degrees, although the peak temperature rise at the start of the run is about seven degrees above ambient. This is in line with literature values. Burgi et al [99] found an empirical formula for the internal temperature rise in a $75 \mu \mathrm{m}$ capillary as $\mathrm{T}=11.5^{*}$ Power/Length $(\mathrm{W} / \mathrm{m})$. The power loss across the depletion region is $I^{2} / \sigma_{d} A$, where $I$ is the current and $\sigma_{d}$ the depletion region conductivity estimated to be $100 \mu \mathrm{S} / \mathrm{cm}$. The temperature rise can then be found from the experimental current. 


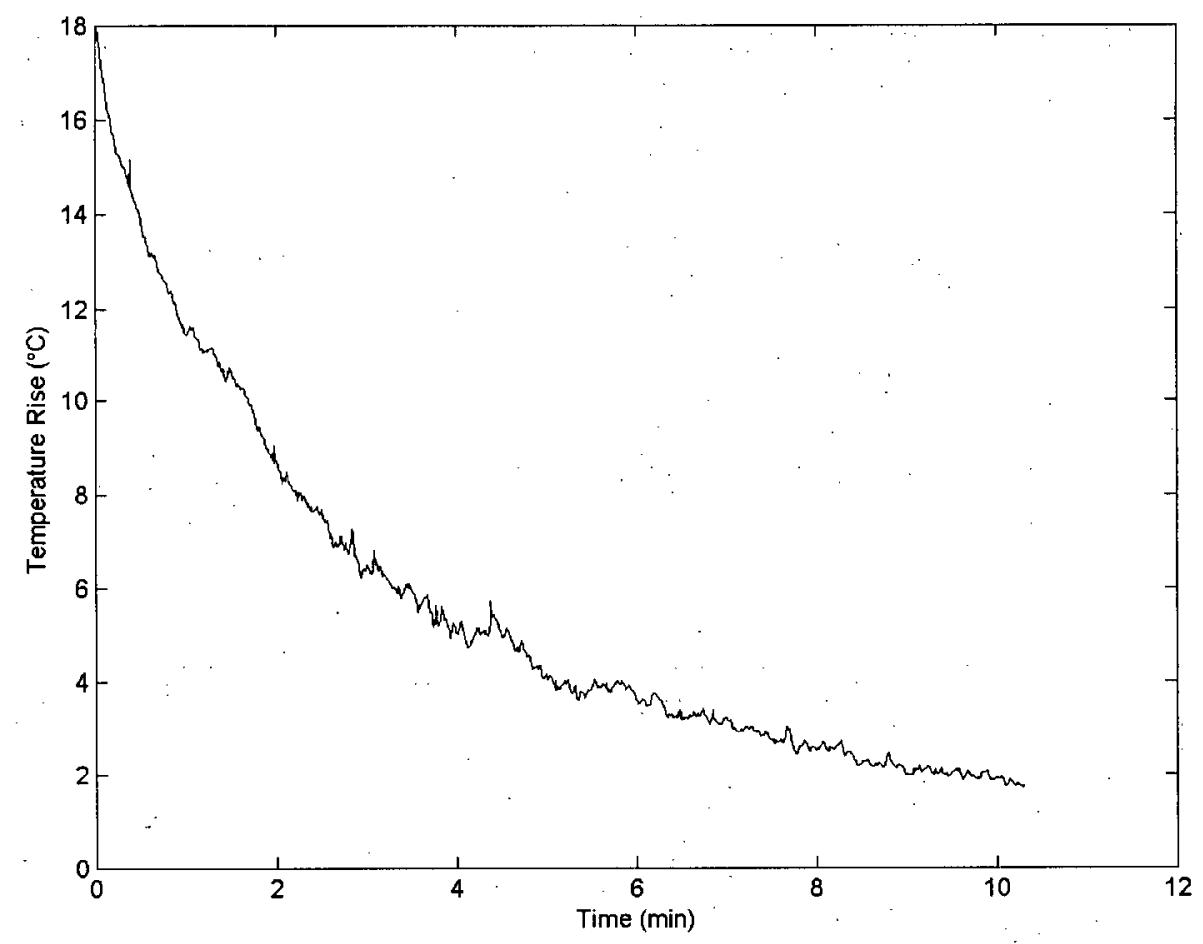

Figure 51 The rise in temperature in the depletion region according to $T=\left.11.5^{\star}\right|^{2} / \sigma_{d} A$, Temperature declines as the current declines.

This is the internal rise in temperature in the capillary. The external rise is less. Both of these results show however that there the temperature rise is not going to make the capillary get much closer to boiling the buffer. As such it is understandable that no spontaneous bubble nucleation takes place. 